\title{
LOCAL CONTROLLABILITY OF A 1-D TANK CONTAINING A FLUID MODELED BY THE SHALLOW WATER EQUATIONS
}

\author{
JEAN-Michel CORON ${ }^{1}$
}

\begin{abstract}
We consider a 1-D tank containing an inviscid incompressible irrotational fluid. The tank is subject to the control which consists of horizontal moves. We assume that the motion of the fluid is well-described by the Saint-Venant equations (also called the shallow water equations). We prove the local controllability of this nonlinear control system around any steady state. As a corollary we get that one can move from any steady state to any other steady state.
\end{abstract}

Mathematics Subject Classification. 76B75, 93B05, 76B15, 35F30.

Received November 12, 2001.

\section{INTRODUCTION}

During the nineties, J.-L. Lions has drawn the attention of mathematicians to flow control. In particular in [14-18], he has obtained various important results and has given several conjectures on the controllability of incompressible fluids. Here we consider a 1-D tank containing an inviscid incompressible irrotational fluid. The tank is subject to one-dimensional horizontal moves. We assume that the horizontal acceleration of the tank is small compared to the gravity constant and that the height of the fluid is small compared to the length of the tank. This motivates the use of the Saint-Venant equations [22] (also called shallow water equations) to describe the motion of the fluid; see e.g. [7] (Sect. 4.2). Hence the dynamics equations considered are (see [8])

$$
\begin{gathered}
H_{t}(t, x)+(H v)_{x}(t, x)=0, \\
v_{t}(t, x)+\left(g H+\frac{v^{2}}{2}\right)_{x}(t, x)=-u(t), \\
v(t, 0)=v(t, L)=0, \\
\frac{\mathrm{d} s}{\mathrm{~d} t}(t)=u(t), \\
\frac{\mathrm{d} D}{\mathrm{~d} t}(t)=s(t),
\end{gathered}
$$

where (see Fig. 1),

- $L$ is the length of the $1-\mathrm{D}$ tank;

- $H(t, x)$ is the height of the fluid at time $t$ and for $x \in[0, L]$;

Keywords and phrases: Controllability, hyperbolic systems, shallow water.

1 Université Paris-Sud, Département de Mathématique, bâtiment 425, 91405 Orsay, France;

e-mail: Jean-Michel.Coron@math.u-psud.fr 
- $v(t, x)$ is the horizontal water velocity of the fluid in a referential attached to the tank at time $t$ and for $x \in[0, L]$ (in the shallow water model, all the points on the same vertical have the same horizontal velocity);

- $u(t)$ is the horizontal acceleration of the tank in the absolute referential;

- $g$ is the gravity constant;

- $s$ is the horizontal velocity of the tank;

- $D$ is the horizontal displacement of the tank.

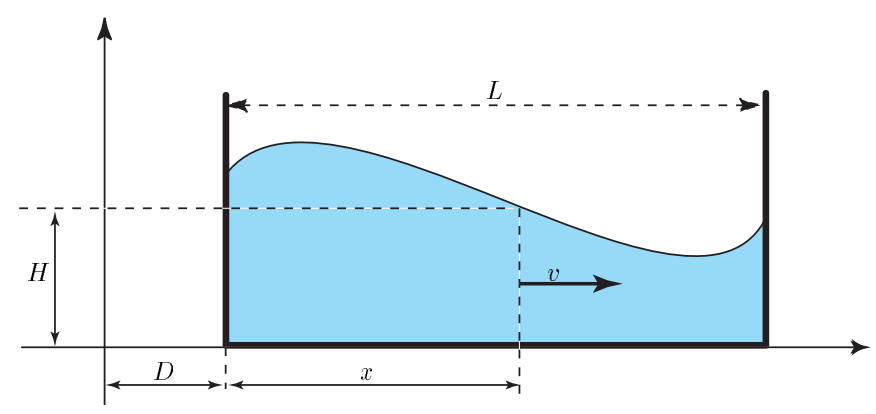

Figure 1. Fluid in the 1-D tank.

This is a control system, denoted $\Sigma$, where

- the state is $Y=(H, v, s, D)$;

- the control is $u \in \mathbb{R}$.

Our goal is to study the local controllability of this control system $\Sigma$ around the equilibrium point

$$
\left(Y_{e}, u_{e}\right):=\left(\left(H_{e}, 0,0,0\right), 0\right)
$$

This problem has been raised in [8]. Of course, the total mass of the fluid is conserved so that, for every solution of (1.1) to $(1.3)$,

$$
\frac{\mathrm{d}}{\mathrm{d} t} \int_{0}^{L} H(t, x) \mathrm{d} x=0 .
$$

(One gets (1.6) by integrating (1.1) on $[0, L]$ and by using (1.3) together with an integration by parts.) Moreover, if $H$ and $v$ are of class $\mathcal{C}^{1}$, it follows from (1.2) and (1.3) that

$$
H_{x}(t, 0)=H_{x}(t, L) \quad(=-u(t) / g)
$$

Therefore we introduce the vector space $E$ of functions $Y=(H, v, s, D) \in \mathcal{C}^{1}([0, L]) \times \mathcal{C}^{1}([0, L]) \times \mathbb{R} \times \mathbb{R}$ such that

$$
\begin{aligned}
H_{x}(0) & =H_{x}(L), \\
v(0) & =v(L)=0,
\end{aligned}
$$

and consider the affine subspace $\mathcal{Y} \subset E$ of $Y=(H, v, s, D) \in E$ satisfying

$$
\int_{0}^{L} H(x) \mathrm{d} x=L H_{e} .
$$


The vector space $E$ is equipped with the usual norm

$$
|Y|:=|H|_{1}+|v|_{1}+|s|+|D|
$$

where, for $w \in \mathcal{C}^{1}([0, L])$,

$$
|w|_{1}:=\operatorname{Max}\left\{|w(x)|+\left|w_{x}(x)\right| ; x \in[0, L]\right\} .
$$

With these notations, we can define a trajectory of the control system $\Sigma$.

Definition 1. Let $T_{1}$ and $T_{2}$ be two real numbers satisfying $T_{1} \leqslant T_{2}$. A function $(Y, u)=((H, v, s, D), u)$ : $\left[T_{1}, T_{2}\right] \rightarrow \mathcal{Y} \times \mathbb{R}$ is a trajectory of the control system $\Sigma$ if

(i) the functions $H$ and $v$ are of class $\mathcal{C}^{1}$ on $\left[T_{1}, T_{2}\right] \times[0, L]$;

(ii) the functions $s$ and $D$ are of class $\mathcal{C}^{1}$ on $\left[T_{1}, T_{2}\right]$ and the function $u$ is continuous on $[0, T]$;

(iii) the equations (1.1) to (1.5) hold for every $(t, x) \in\left[T_{1}, T_{2}\right] \times[0, L]$.

Our main result states that the control system $\Sigma$ is locally controllable around the equilibrium point $\left(Y_{e}, u_{e}\right)$. More precisely, one has the following theorem:

Theorem 2. There exists $T>0, C_{0}>0$ and $\eta>0$ such that, for every $Y_{0}=\left(H_{0}, v_{0}, s_{0}, D_{0}\right) \in \mathcal{Y}$, and for every $Y_{1}=\left(H_{1}, v_{1}, s_{1}, D_{1}\right) \in \mathcal{Y}$ such that

$$
\left|H_{0}-H_{e}\right|_{1}+\left|v_{0}\right|_{1}<\eta,\left|H_{1}-H_{e}\right|_{1}+\left|v_{1}\right|_{1}<\eta,\left|s_{1}-s_{0}\right|+\left|D_{1}-s_{0} T-D_{0}\right|<\eta,
$$

there exists a trajectory $(Y, u):[0, T] \rightarrow \mathcal{Y} \times \mathbb{R}, t \mapsto((H(t), v(t), s(t), D(t)), u(t))$ of the control system $\Sigma$ such that

$$
Y(0)=Y_{0} \text { and } Y(T)=Y_{1},
$$

and, for every $t \in[0, T]$,

$$
\left|H(t)-H_{e}\right|_{1}+|v(t)|_{1}+|u(t)|<C_{0}\left(\sqrt{\left|H_{0}-H_{e}\right|_{1}+\left|v_{0}\right|_{1}+\left|H_{1}-H_{e}\right|_{1}+\left|v_{1}\right|_{1}}+\left|s_{1}-s_{0}\right|+\left|D_{1}-s_{0} T-D_{0}\right|\right) .
$$

As a corollary of this theorem, any steady state $Y_{1}=\left(H_{e}, 0,0, D_{1}\right)$ can be reached from any other steady state $Y_{0}=\left(H_{e}, 0,0, D_{0}\right)$. More precisely, one has the following corollary:

Corollary 3. Let $T, C_{0}$ and $\eta$ be as in Theorem 2. Let $D_{0}$ and $D_{1}$ be two real numbers and let $k$ be a positive integer such that $\left|D_{1}-D_{0}\right|<k \eta$. Then, there exists a trajectory $(Y, u):[0, k T] \rightarrow \mathcal{Y} \times \mathbb{R}, t \mapsto$ $((H(t), v(t), s(t), D(t)), u(t))$ of the control system $\Sigma$ such that

$$
\begin{gathered}
Y(0)=\left(H_{e}, 0,0, D_{0}\right) \text { and } Y(k T)=\left(H_{e}, 0,0, D_{1}\right), \\
\left|H(t)-H_{e}\right|_{1}+|v(t)|_{1}+|u(t)|<C_{0} \frac{\left|D_{1}-D_{0}\right|}{k} \forall t \in[0, k T] .
\end{gathered}
$$

Indeed, it suffices to apply Theorem 2 with $Y_{0}=\left(H_{e}, 0,0, D_{0}+(i / k)\left(D_{1}-D_{0}\right)\right)$ and $Y_{1}=\left(H_{e}, 0,0, D_{0}\right.$ $\left.+((i+1) / k)\left(D_{1}-D_{0}\right)\right)$ for $i \in\{0, \ldots, k-1\}$.

In Section 2 we give the steps of the proof of Theorem 2. This proof relies on two propositions (Props. 6 and 5), whose demonstration are given in Sections 3 and 4 respectively. 


\section{Sketch of the proof of Theorem 2}

Let us first point out that by scaling arguments one can assume without loss of generality that

$$
L=g=H_{e}=1 .
$$

Indeed, if we let

$$
\begin{gathered}
H^{*}(t, x):=\frac{1}{H_{e}} H\left(\frac{L t}{\sqrt{H_{e} g}}, L x\right), v^{*}(t, x):=\frac{1}{\sqrt{H_{e} g}} v\left(\frac{L t}{\sqrt{H_{e} g}}, L x\right), \\
u^{*}(t):=\frac{L}{H_{e} g} u\left(\frac{L t}{\sqrt{H_{e} g}}\right), s^{*}(t):=\frac{1}{\sqrt{H_{e} g}} s\left(\frac{L t}{\sqrt{H_{e} g}}\right), D^{*}(t):=\frac{1}{L} D\left(\frac{L t}{\sqrt{H_{e} g}}\right),
\end{gathered}
$$

with $x \in[0,1]$, then equations $(1.1)$ to $(1.5)$ are equivalent to

$$
\begin{gathered}
H_{t}^{*}(t, x)+\left(H^{*} v^{*}\right)_{x}(t, x)=0, \\
v_{t}^{*}(t, x)+\left(H^{*}+\frac{v^{* 2}}{2}\right)_{x}(t, x)=-u^{*}(t), \\
v^{*}(t, 0)=v^{*}(t, 1)=0, \\
\frac{\mathrm{d} s^{*}}{\mathrm{~d} t}(t)=u^{*}(t), \\
\frac{\mathrm{d} D^{*}}{\mathrm{~d} t}(t)=s^{*}(t) .
\end{gathered}
$$

From now on, we always assume that we have (2.1). Since $(Y, u)=((H, v, s, D), u)$ is a trajectory of the control system $\Sigma$ if and only if $((H, v, s-a, D-a t-b), u)$ is a trajectory of the control system $\Sigma$, we may assume without loss of generality that $s_{0}=D_{0}=0$.

The proof of Theorem 2 relies on the return method, a method introduced in [1] in order to solve a stabilization problem, and used in $[2,3,5,9-11,24]$ for controllability problems. Roughly speaking, the return method consists in looking for a trajectory $(\bar{Y}, \bar{u}):[0, T] \rightarrow \mathcal{Y} \times \mathbb{R}$ of the control system $\Sigma$ satisfying

$$
\bar{Y}(0)=\bar{Y}(T)=Y_{e},
$$

the linearized control system around $(\bar{Y}, \bar{u})$ is controllable.

If such a trajectory $(\bar{Y}, \bar{u})$ exists, one can often (this is always the case for finite dimensional control system) prove that, for every $Y_{0}$ and $Y_{1}$ close to $Y_{e}$, there exists a trajectory $(Y, u)$ close to $(\bar{Y}, \bar{u})$ such that

$$
Y(0)=Y_{0} \text { and } Y(T)=Y_{1} .
$$

Let us point out that, as already noticed in [8], property $(2.3)$ does not hold for the natural trajectory $(\bar{Y}, \bar{u})$ $=\left(Y_{e}, u_{e}\right)$. Indeed the linearized control system around $\left(Y_{e}, u_{e}\right)$ is

$$
\left(\Sigma_{0}\right)\left\{\begin{array}{l}
h_{t}+v_{x}=0, \\
v_{t}+h_{x}=-u(t), \\
v(t, 0)=v(t, 1)=0, \\
\frac{\mathrm{d} s}{\mathrm{~d} t}(t)=u(t), \\
\frac{\mathrm{d} D}{\mathrm{~d} t}(t)=s(t),
\end{array}\right.
$$


where the state is $(h, v, s, D) \in \mathcal{Y}_{0}$, with

$$
\mathcal{Y}_{0}:=\left\{(h, v, s, D) \in E ; \int_{0}^{L} h \mathrm{~d} x=0\right\}
$$

and the control is $u \in \mathbb{R}$. But (2.4) implies that, if

$$
h(0,1-x)=-h(0, x) \text { and } v(0,1-x)=v(0, x) \forall x \in[0,1],
$$

then

$$
h(t, 1-x)=-h(t, x) \text { and } v(t, 1-x)=v(t, x) \forall x \in[0,1], \forall t .
$$

Remark 4. Even if the control system (2.4) is not controllable, one can move, as it is proved in [8], from any steady state $\left(h_{0}, v_{0}, s_{0}, D_{0}\right):=\left(0,0,0, D_{0}\right)$ to any steady state $\left(h_{1}, v_{1}, s_{1}, D_{1}\right):=\left(0,0,0, D_{1}\right)$ for this control system (see also [21] when the tank has a non-straight bottom). This does not imply that the related property (move from $\left(1,0,0, D_{0}\right)$ to $\left.\left(1,0,0, D_{1}\right)\right)$ also holds for the nonlinear control system $\Sigma$, but it follows from Corollary 3 , that this property indeed also holds for the nonlinear control system $\Sigma$. Moreover the fact that, for the control system (2.4), it is possible [8], to move from any steady state $\left(h_{0}, v_{0}, s_{0}, D_{0}\right):=\left(0,0, s_{0}, D_{0}\right)$ to any steady state $\left(h_{1}, v_{1}, s_{1}, D_{1}\right):=\left(0,0, s_{1}, D_{1}\right)$ explains why in the right hand side of (1.12) one has $\left|s_{1}-s_{0}\right|+\left|D_{1}-s_{0} T-D_{0}\right|$ and not $\left(\left|s_{1}-s_{0}\right|+\left|D_{1}-s_{0} T-D_{0}\right|\right)^{1 / 2}$.

As in $[1-3,5,9-11,24]$ one has to look for more complicated trajectories $(\bar{Y}, \bar{u})$ in order to have $(2.3)$. In fact, as in [4], one can require instead of (2.2), the weaker property

$$
\bar{Y}(0)=Y_{e} \text { and } \bar{Y}(T) \text { is close to } Y_{e}
$$

and hope that, as it happens in [4], the controllability around $(\bar{Y}, \bar{u})$ will be strong enough to tackle the problem that $\bar{Y}(T)$ is not $Y_{e}$ but only close to $Y_{e}$. Moreover, since as it is proved in [8], one can move for the linear control system $\Sigma_{0}$, from $y_{e}=(0,0,0,0)$ to $\left(0,0, s_{1}, D_{1}\right)$, it is natural to try not to "return" to $Y_{e}$, but requires instead (2.5) the property

$$
\bar{Y}(0)=Y_{e} \text { and } \bar{Y}(T) \text { is close to }\left(1,0, s_{1}, D_{1}\right) .
$$

In order to use this method, one first needs to have trajectories of the control system $\Sigma$ such that the linearized control system around these trajectories are controllable. Let us give an example of a family of such trajectories. Let us fix a positive real number $T^{*}$ in $(2,+\infty)$. For $\gamma \in(0,1]$ and $(a, b) \in \mathbb{R}^{2}$, let us define $\left(Y^{\gamma, a, b}, u^{\gamma}\right)$ : $\left[0, T^{*}\right] \rightarrow \mathcal{Y} \times \mathbb{R}$ by

$$
\begin{gathered}
Y^{\gamma, a, b}(t, x):=\left(1+\gamma\left(\frac{1}{2}-x\right), 0, \gamma t+a, \gamma \frac{t^{2}}{2}+a t+b\right) \forall t \in\left[0, T^{*}\right], \forall x \in[0,1], \\
u^{\gamma}(t):=\gamma \forall t \in\left[0, T^{*}\right] .
\end{gathered}
$$


Then, $\left(Y^{\gamma, a, b}, u^{\gamma}\right)$ is a trajectory of the control system $\Sigma$. The linearized control system around this trajectory is the following control system

$$
\left(\Sigma_{\gamma}\right)\left\{\begin{array}{l}
h_{t}+\left(\left(1+\gamma\left(\frac{1}{2}-x\right)\right) v\right)_{x}=0 \\
v_{t}+h_{x}=-u(t) \\
v(t, 0)=v(t, 1)=0 \\
\frac{\mathrm{d} s}{\mathrm{~d} t}(t)=u(t) \\
\frac{\mathrm{d} D}{\mathrm{~d} t}(t)=s(t)
\end{array}\right.
$$

where the state is $(h, v, s, D) \in \mathcal{Y}_{0}$ and the control is $u \in \mathbb{R}$. As we shall see in Section 4 below, this linear control system $\Sigma_{\gamma}$ is controllable if $\gamma>0$ is small enough. Unfortunately the controllability of $\Sigma_{\gamma}$ does not seem to imply directly the local controllability of the control system $\Sigma$ around the trajectory $\left(Y^{\gamma, a, b}, u^{\gamma}\right)$. Indeed the map from $\mathcal{Y} \times \mathcal{C}^{0}([0, T])$ into $\mathcal{Y}$ which associates to any initial data $Y_{0}=\left(H_{0}, v_{0}, s_{0}, D_{0}\right) \in \mathcal{Y}$ and to any $u \in \mathcal{C}^{0}([0, T])$ such that

$$
H_{0 x}(0)=H_{0 x}(1)=-u(0)
$$

the state $Y(T) \in \mathcal{Y}$, where $Y=(H, v, s, D):[0, T] \rightarrow \mathcal{Y}$ satisfies $(1.1)$ to $(1.5)$ and $Y(0)=Y_{0}$ is well-defined and continuous on a small open neighborhood of $\left(Y_{e}, 0\right)$ (see e.g. [19]) but is not of class $\mathcal{C}^{1}$ on this neighborhood. So one cannot use the classical inverse function theorem to get the desired local controllability. To take care of this problem, one adapts the usual iterative scheme used to prove the existence of solutions to hyperbolic systems (see e.g. [6], pp. 476-478, [12], pp. 54-55, [19], pp. 96-107, [20], pp. 35-43 or [23], pp. 106-116) see also $[2,3,5,9-11]$ for the Euler and the Navier control system for incompressible fluids): one uses the following inductive procedure $\left(h^{n}, v^{n}, s^{n}, D^{n}, u^{n}\right) \mapsto\left(h^{n+1}, v^{n+1}, s^{n+1}, D^{n+1}, u^{n+1}\right)$ so that

$$
\begin{gathered}
h_{t}^{n+1}+v^{n} h_{x}^{n+1}+\left(1+\gamma\left(\frac{1}{2}-x\right)+h^{n}\right) v_{x}^{n+1}-\gamma v^{n+1}=0 \\
v_{t}^{n+1}+h_{x}^{n+1}+v^{n} v_{x}^{n+1}=-u^{n+1}(t) \\
v^{n+1}(t, 0)=v^{n+1}(t, L)=0, \\
\frac{\mathrm{d} s^{n+1}}{\mathrm{~d} t}(t)=u^{n+1}(t), \\
\frac{\mathrm{d} D^{n+1}}{\mathrm{~d} t}(t)=s^{n+1}(t),
\end{gathered}
$$

and $\left(h^{n+1}, v^{n+1}, s^{n+1}, D^{n+1}, u^{n+1}\right)$ has the required value for $t=0$ and for $t=T^{*}$. Unfortunately we have only been able to prove that the control system $(2.10,2.14)$, where the state is $\left(h^{n+1}, v^{n+1}, s^{n+1}, D^{n+1}\right)$ and the control is $u^{n+1}$, is controllable under a special assumption on $\left(h^{n}, v^{n}\right)$. (This assumption is that $\theta$, defined in (4.38), is 0 see Rem. 26 below.) Hence one has to insure that, at each iterative step, $\left(h^{n}, v^{n}\right)$ satisfies this condition, which turns out to be possible. So one gets the following proposition, which is proved in Section 4.

Proposition 5. There exist $C_{1}>0, \mu>0$ and $\gamma_{0} \in(0,1]$ such that, for every $\gamma \in\left[0, \gamma_{0}\right]$, for every $(a, b) \in \mathbb{R}^{2}$ and for every $\left(Y_{0}, Y_{1}\right) \in \mathcal{Y}^{2}$ satisfying

$$
\left|Y_{0}-Y^{\gamma, a, b}(0)\right| \leqslant \mu \gamma^{2} \text { and }\left|Y_{1}-Y^{\gamma, a, b}\left(T^{*}\right)\right| \leqslant \mu \gamma^{2},
$$


there exists a trajectory $(Y, u):\left[0, T^{*}\right] \rightarrow \mathcal{Y} \times \mathbb{R}$ of the control system $\Sigma$ such that

$$
\begin{gathered}
Y(0)=Y_{0} \text { and } Y\left(T^{*}\right)=Y_{1} \\
\left|Y(t)-Y^{\gamma, a, b}(t)\right|+|u(t)| \leqslant C_{1} \gamma \forall t \in\left[0, T^{*}\right]
\end{gathered}
$$

One now needs to construct, for every given $\gamma>0$ small enough, trajectories $(Y, u):\left[0, T^{0}\right] \rightarrow \mathcal{Y} \times \mathbb{R}$ of the control system $\Sigma$ satisfying

$$
Y(0)=(1,0,0,0) \text { and }\left|Y\left(T^{0}\right)-Y^{\gamma, a, b}(0)\right| \leqslant \mu \gamma^{2}
$$

and trajectories $(Y, u):\left[T^{0}+T^{*}, T^{0}+T^{*}+T^{1}\right] \rightarrow \mathcal{Y} \times \mathbb{R}$ of the control system $\Sigma$ such that

$$
Y\left(T^{0}+T^{1}+T^{*}\right)=\left(1,0, s_{1}, D_{1}\right) \text { and }\left|Y\left(T^{0}+T^{*}\right)-Y^{\gamma, a, b}\left(T^{*}\right)\right| \leqslant \mu \gamma^{2},
$$

for suitable choice of $(a, b) \in \mathbb{R}^{2}, T^{0}>0, T^{1}>0$. Let us first point out that it follows from [8] that one knows explicit trajectories $\left(Y^{l}, u^{l}\right):\left[0, T^{0}\right] \rightarrow \mathcal{Y} \times \mathbb{R}$ of the linearized control system around $(0,0)$ satisfying $Y^{l}(0)=0$ and $Y^{l}\left(T^{0}\right)=Y^{\gamma, a, b}(0)$. Then, the idea is that, if one moves "slowly", the same control $u^{l}$ gives a trajectory $(Y, u):\left[0, T^{0}\right] \rightarrow \mathcal{Y} \times \mathbb{R}$ of the control system $\Sigma$ such that $(2.16)$ holds. More precisely, let $f_{0} \in \mathcal{C}^{4}([0,4])$ be such that

$$
\begin{gathered}
f_{0}=0 \text { in }[0,1 / 2] \cup[3,4], \\
f_{0}(t)=s / 2 \forall t \in[1,3 / 2], \\
\int_{0}^{4} f_{0}\left(t_{1}\right) \mathrm{d} t_{1}=0 .
\end{gathered}
$$

Similarly, let $f_{1} \in \mathcal{C}^{4}([0,4])$ and $f_{2} \in \mathcal{C}^{4}([0,4])$ be such that

$$
\begin{gathered}
f_{1}=0 \text { in }[0,1 / 2] \cup[1,3 / 2] \text { and } f_{1}=1 / 2 \text { in }[3,4], \\
\int_{0}^{3} f_{1}\left(t_{1}\right) \mathrm{d} t_{1}=0, \\
f_{2}=0 \text { in }[0,1 / 2] \cup[1,3 / 2] \cup[3,4], \\
\int_{0}^{4} f_{2}\left(t_{1}\right) \mathrm{d} t_{1}=1 / 2 .
\end{gathered}
$$

Let

$$
\mathbb{D}:=\left\{(\bar{s}, \bar{D}) \in \mathbb{R}^{2} ;|\bar{s}| \leqslant 1,|\bar{D}| \leqslant 1\right\}
$$

For $(\bar{s}, \bar{D}) \in \mathbb{D}$, let $f_{\bar{s}, \bar{D}} \in \mathcal{C}^{4}([0,4])$ be defined by

$$
f_{\bar{s}, \bar{D}}:=f_{0}+\bar{s} f_{1}+\bar{D} f_{2}
$$

For $\epsilon \in(0,1 / 2]$ and for $\gamma \in \mathbb{R}$, let $u_{\bar{s}, \bar{D}}^{\epsilon, \gamma}:[0,3 / \epsilon] \rightarrow \mathbb{R}$ be defined by

$$
u_{\bar{s}, \bar{D}}^{\epsilon, \gamma}(t):=\gamma f_{\bar{s}, \bar{D}}^{\prime}(\epsilon t)+\gamma f_{\bar{s}, \bar{D}}^{\prime}(\epsilon(t+1)) .
$$


Let $\left(h_{\bar{s}, \bar{D}}^{\epsilon, \gamma}, v_{\bar{s}, \bar{D}}^{\epsilon, \gamma}, s_{\bar{s}, \bar{D}}^{\epsilon, \gamma}, D_{\bar{s}, \bar{D}}^{\epsilon, \gamma}\right):[0,3 / \epsilon] \rightarrow \mathcal{C}^{1}([0,1]) \times \mathcal{C}^{1}([0,1]) \times \mathbb{R} \times \mathbb{R}$ be such that $(2.4)$ holds for $(h, v, s, D)$ $=\left(h_{\bar{s}, \bar{D}}^{\epsilon, \gamma}, v_{\bar{s}, \bar{D}}^{\epsilon, \gamma}, s_{\bar{s}, \bar{D}}^{\epsilon, \gamma}, D_{\bar{s}, \bar{D}}^{\epsilon, \gamma}\right), u=u_{\bar{s}, \bar{D}}^{\epsilon, \gamma}$ and

$$
\left(h_{\bar{s}, \bar{D}}^{\epsilon, \gamma}(0, \cdot), v_{\bar{s}, \bar{D}}^{\epsilon, \gamma}(0, \cdot), s_{\bar{s}, \bar{D}}^{\epsilon, \gamma}(0), D_{\bar{s}, \bar{D}}^{\epsilon, \gamma}(0)\right)=(0,0,0,0)
$$

From [8] one gets that

$$
\begin{gathered}
h_{\bar{s}, \bar{D}}^{\epsilon, \gamma}(t, x)=-\frac{\gamma}{\epsilon} f_{\bar{s}, \bar{D}}(\epsilon(t+x))+\frac{\gamma}{\epsilon} f_{\bar{s}, \bar{D}}(\epsilon(t+1-x)), \\
v_{\bar{s}, \bar{D}}^{\epsilon, \gamma}(t, x)=\frac{\gamma}{\epsilon} f_{\bar{s}, \bar{D}}(\epsilon(t+x))+\frac{\gamma}{\epsilon} f_{\bar{s}, \bar{D}}(\epsilon(t+1-x))-\frac{\gamma}{\epsilon} f_{\bar{s}, \bar{D}}(\epsilon t)-\frac{\gamma}{\epsilon} f_{\bar{s}, \bar{D}}(\epsilon(t+1)), \\
s_{\bar{s}, \bar{D}}^{\epsilon, \gamma}(t)=\frac{\gamma}{\epsilon} f_{\bar{s}, \bar{D}}(\epsilon t)+\frac{\gamma}{\epsilon} f_{\bar{s}, \bar{D}}(\epsilon(t+1)), \\
D_{\bar{s}, \bar{D}}^{\epsilon, \gamma}(t)=\frac{\gamma}{\epsilon^{2}} F_{\bar{s}, \bar{D}}(\epsilon t)+\frac{\gamma}{\epsilon^{2}} F_{\bar{s}, \bar{D}}(\epsilon(t+1)),
\end{gathered}
$$

with

$$
F_{\bar{s}, \bar{D}}(t):=\int_{0}^{t} f_{\bar{s}, \bar{D}}\left(t_{1}\right) \mathrm{d} t_{1} .
$$

In particular, using also (2.18) to (2.24), one gets

$$
\begin{gathered}
h_{\bar{s}, \bar{D}}^{\epsilon, \gamma}\left(\frac{1}{\epsilon}+t, x\right)=\gamma\left(\frac{1}{2}-x\right) \text { and } v_{\bar{s}, \bar{D}}^{\epsilon, \gamma}\left(\frac{1}{\epsilon}+t, x\right)=0 \forall t \in\left[0, \frac{1-2 \epsilon}{2 \epsilon}\right], \forall x \in[0,1], \\
s_{\bar{s}, \bar{D}}^{\epsilon, \gamma}\left(\frac{1}{\epsilon}+t\right)=\frac{\gamma}{\epsilon}+\frac{\gamma}{2}+\gamma t, D_{\bar{s}, \bar{D}}^{\epsilon, \gamma}\left(\frac{1}{\epsilon}+t\right)=D_{\bar{s}, \bar{D}}^{\epsilon, \gamma}\left(\frac{1}{\epsilon}\right)+\left(\frac{\gamma}{\epsilon}+\frac{\gamma}{2}\right) t+\frac{\gamma}{2} t^{2} \forall t \in\left[0, \frac{1-2 \epsilon}{2 \epsilon}\right], \\
h_{\bar{s}, \bar{D}}^{\epsilon, \gamma}\left(\frac{3}{\epsilon}, x\right)=0 \text { and } v_{\bar{s}, \bar{D}}^{\epsilon, \gamma}\left(\frac{3}{\epsilon}, x\right)=0 \forall x \in[0,1], \\
s_{\bar{s}, \bar{D}}^{\epsilon, \gamma}\left(\frac{3}{\epsilon}\right)=\frac{\gamma}{\epsilon} \bar{s} \text { and } D_{\bar{s}, \bar{D}}^{\epsilon, \gamma}\left(\frac{3}{\epsilon}\right)=\frac{\gamma}{2 \epsilon} \bar{s}+\frac{\gamma}{\epsilon^{2}} \bar{D} .
\end{gathered}
$$

Let $H_{\bar{s}, \bar{D}}^{\epsilon, \gamma}=1+h_{\bar{s}, \bar{D}}^{\epsilon, \gamma}$ and $Y_{\bar{s}, \bar{D}}^{\epsilon, \gamma}=\left(H_{\bar{s}, \bar{D}}^{\epsilon, \gamma}, v_{\bar{s}, \bar{D}}^{\epsilon, \gamma}, s_{\bar{s}, \bar{D}}^{\epsilon, \gamma}, D_{\bar{s}, \bar{D}}^{\epsilon, \gamma}\right)$. Consider

$$
a_{\epsilon, \gamma}:=\frac{\gamma}{\epsilon} f_{\bar{s}, \bar{D}}(1)+\frac{\gamma}{\epsilon} f_{\bar{s}, \bar{D}}(1+\epsilon)=\frac{\gamma}{\epsilon}+\frac{\gamma}{2}, b_{\epsilon, \gamma}^{\bar{s}, \bar{D}}:=\frac{\gamma}{\epsilon^{2}} F_{\bar{s}, \bar{D}}(1)+\frac{\gamma}{\epsilon^{2}} F_{\bar{s}, \bar{D}}(1+\epsilon)=D_{\bar{s}, \bar{D}}^{\epsilon, \gamma}\left(\frac{1}{\epsilon}\right) .
$$

Using $(2.7,2.31)$ and $(2.32)$, one has

$$
Y_{\bar{s}, \bar{D}}^{\epsilon, \gamma}\left(\frac{1}{\epsilon}\right)=Y^{\gamma, a_{\epsilon, \gamma}, b_{\epsilon, \gamma}^{\bar{s}, \bar{D}}}(0, \cdot)
$$

and, if $\epsilon \in\left(0,1 /\left(2\left(T^{*}+1\right)\right)\right]$,

$$
Y_{\bar{s}, \bar{D}}^{\epsilon, \gamma}\left(\frac{1}{\epsilon}+T^{*}\right)=Y^{\gamma, a_{\epsilon, \gamma}, b_{\epsilon, \gamma}^{\bar{s}, \bar{D}}}\left(T^{*}\right)
$$

The next proposition shows that one can achieve (2.16) with $u=u_{\bar{s}, \bar{D}}^{\epsilon, \gamma}$ for suitable choices of $T^{0}, \epsilon$ and $\gamma$. 
Proposition 6. There exists a constant $C_{2}>2$ such that, for every $\epsilon \in\left(0,1 / C_{2}\right]$, for every $(\bar{s}, \bar{D}) \in \mathbb{D}$ and for every $\gamma \in\left[0, \epsilon / C_{2}\right]$, there exists one and only one map $\tilde{Y}_{\bar{s}, \bar{D}}^{\epsilon, \gamma}:[0,1 / \epsilon] \rightarrow \mathcal{Y}$ satisfying the two following conditions

$$
\begin{gathered}
\left(\tilde{Y}_{\bar{s}, \bar{D}}^{\epsilon, \gamma}, u_{\bar{s}, \bar{D}}^{\epsilon, \gamma}\right) \text { is a trajectory of the control system } \Sigma(\text { on }[0,1 / \epsilon]) \\
\tilde{Y}_{\bar{s}, \bar{D}}^{\epsilon, \gamma}(0)=(1,0,0,0)
\end{gathered}
$$

and this unique map $\tilde{Y}_{\bar{s}, \bar{D}}^{\epsilon, \gamma}$ verifies

$$
\left|\tilde{Y}_{\bar{s}, \bar{D}}^{\epsilon, \gamma}(t)-Y_{\bar{s}, \bar{D}}^{\epsilon, \gamma}(t)\right| \leqslant C_{2} \epsilon \gamma^{2} \forall t \in[0,1 / \epsilon]
$$

In particular, by (2.31),

$$
\left|\tilde{v}_{\bar{s}, \bar{D}}^{\epsilon, \gamma}\left(\frac{1}{\epsilon}\right)\right|_{1}+\left|\tilde{h}_{\bar{s}, \bar{D}}^{\epsilon, \gamma}\left(\frac{1}{\epsilon}\right)-\gamma\left(\frac{1}{2}-x\right)\right|_{1} \leqslant C_{2} \epsilon \gamma^{2}
$$

Similarly, one has the following proposition, which shows that (2.17) is achieved with $u=u_{\bar{s}, \bar{D}}^{\epsilon, \gamma}$ for suitable choices of $T^{1}, \epsilon$ and $\gamma$.

Proposition 7. There exists a constant $C_{3}>2\left(T^{*}+1\right)$ such that, for every $\epsilon \in\left(0,1 / C_{3}\right]$, for every $(\bar{s}, \bar{D}) \in \mathbb{D}$, and for every $\gamma \in\left[0, \epsilon / C_{3}\right]$, there exists one and only one map $\hat{Y}_{\bar{s}, \bar{D}}^{\epsilon, \gamma}:\left[(1 / \epsilon)+T^{*}, 3 / \epsilon\right] \rightarrow \mathcal{Y}$ satisfying the two following conditions

$$
\begin{gathered}
\left(\hat{Y}_{\bar{s}, \bar{D}}^{\epsilon, \gamma}, u_{\bar{s}, \bar{D}}^{\epsilon, \gamma}\right) \text { is a trajectory of the control system } \Sigma\left(\text { on }\left[(1 / \epsilon)+T^{*}, 3 / \epsilon\right]\right), \\
\hat{Y}_{\bar{s}, \bar{D}}^{\epsilon, \gamma}\left(\frac{3}{\epsilon}\right)=\left(1,0, \frac{\gamma}{\epsilon} \bar{s}, \frac{\gamma}{2 \epsilon} \bar{s}+\frac{\gamma}{\epsilon^{2}} \bar{D}\right)=Y_{\bar{s}, \bar{D}}^{\epsilon, \gamma}\left(\frac{3}{\epsilon}\right)
\end{gathered}
$$

and this unique map $\hat{Y}^{\epsilon, \gamma}$ verifies

$$
\left|\hat{Y}_{\bar{s}, \bar{D}}^{\epsilon, \gamma}(t)-Y_{\bar{s}, \bar{D}}^{\epsilon, \gamma}(t)\right| \leqslant C_{3} \epsilon \gamma^{2} \forall t \in\left[(1 / \epsilon)+T^{*}, 3 / \epsilon\right]
$$

In particular, by (2.31),

$$
\left|\hat{v}_{\bar{s}, \bar{D}}^{\epsilon, \gamma}\left(\frac{1}{\epsilon}\right)\right|_{1}+\left|\hat{h}_{\bar{s}, \bar{D}}^{\epsilon, \gamma}\left(\frac{1}{\epsilon}\right)-\gamma\left(\frac{1}{2}-x\right)\right|_{1} \leqslant C_{2} \epsilon \gamma^{2}
$$

Let us choose

$$
\epsilon:=\operatorname{Min}\left(\frac{1}{C_{2}}, \frac{1}{C_{3}}, \frac{\mu}{2 C_{2}}, \frac{\mu}{2 C_{3}}\right) \leqslant \frac{1}{2} .
$$

Let us point out that there exists $C_{4}>0$ such that, for every $(\bar{s}, \bar{D}) \in \mathbb{D}$ and for every $\gamma \in[-\epsilon, \epsilon]$,

$$
\left|H_{\bar{s}, \bar{D}}^{\epsilon, \gamma}\right|_{\mathcal{C}^{2}([0,3 / \epsilon] \times[0,1])}+\left|v_{\bar{s}, \bar{D}}^{\epsilon, \gamma}\right|_{\mathcal{C}^{2}([0,3 / \epsilon] \times[0,1])} \leqslant C_{4},
$$

which, with straightforward estimates, leads to the next proposition, whose proof is omitted. 
Proposition 8. There exists $C_{5}>0$ such that, for every $(\bar{s}, \bar{D}) \in \mathbb{D}$, for every $Y_{0}=\left(H_{0}, v_{0}, s_{0}, D_{0}\right) \in \mathcal{Y}$ with

$$
\left|Y_{0}-Y_{e}\right| \leqslant \frac{1}{C_{5}}, s_{0}=0, D_{0}=0
$$

and for every $\gamma \in\left[0, \epsilon / C_{2}\right]$, there exists one and only one $Y:[0,1 / \epsilon] \rightarrow \mathcal{Y}$ such that

$$
\begin{gathered}
\left(Y, u_{\bar{s}, \bar{D}}^{\varepsilon, \gamma}-H_{0 x}(0)\right) \text { is a trajectory of the control system } \Sigma, \\
Y(0)=Y_{0},
\end{gathered}
$$

and this unique map $Y$ satisfies

$$
\left|Y(t)-\tilde{Y}_{\bar{s}, \bar{D}}^{\varepsilon, \gamma}(t)\right| \leqslant C_{5}\left|Y_{0}-Y_{e}\right|, \forall t \in[0,1 / \epsilon] .
$$

Similarly, equation (2.42) leads to the following proposition:

Proposition 9. There exists $C_{6}>0$ such that, for every $(\bar{s}, \bar{D}) \in \mathbb{D}$, for every $\gamma \in\left[0, \epsilon / C_{3}\right]$, and for every $Y_{1}=\left(H_{1}, v_{1}, s_{1}, D_{1}\right) \in \mathcal{Y}$ such that

$$
\left|Y_{1}-\left(1,0, \frac{\gamma}{\epsilon} \bar{s}, \frac{\gamma}{2 \epsilon} \bar{s}+\frac{\gamma}{\epsilon^{2}} \bar{D}\right)\right| \leqslant \frac{1}{C_{6}}, s_{1}=\frac{\gamma}{\epsilon} \bar{s}, D_{1}=\frac{\gamma}{2 \epsilon} \bar{s}+\frac{\gamma}{\epsilon^{2}} \bar{D}
$$

there exists one and only one $Y:\left[(1 / \epsilon)+T^{*}, 3 / \epsilon\right] \rightarrow \mathcal{Y}$ such that

$$
\begin{gathered}
\left(Y, u_{\bar{s}, \bar{D}}^{\varepsilon, \gamma}-H_{1 x}(0)\right) \text { is a trajectory of the control system } \Sigma \\
Y(3 / \epsilon)=Y_{1},
\end{gathered}
$$

and this unique map $Y$ satisfies

$$
\left|Y(t)-\hat{Y}_{\bar{s}, \bar{D}}^{\varepsilon, \gamma}(t)\right| \leqslant C_{6}\left|Y_{1}-Y_{\bar{s}, \bar{D}}^{\varepsilon, \gamma}(3 / \epsilon)\right|, \forall t \in\left[(1 / \epsilon)+T^{*}, 3 / \epsilon\right] .
$$

Finally define

$$
\begin{gathered}
T:=\frac{3}{\epsilon}, \\
\eta:=\operatorname{Min}\left(\frac{\epsilon^{2} \mu}{2 C_{5}\left(C_{3}^{2}+C_{2}^{2}\right)}, \frac{\epsilon^{2} \mu}{2 C_{6}\left(C_{3}^{2}+C_{2}^{2}\right)}, \frac{\epsilon}{C_{2}}, \frac{\epsilon}{C_{3}}, \frac{1}{C_{5}}, \frac{1}{C_{6}}, \frac{\gamma_{0}^{2} \mu}{2 C_{5}}, \frac{\gamma_{0}^{2} \mu}{2 C_{6}}, \gamma_{0}\right) .
\end{gathered}
$$

We want to check that Theorem 2 holds with these constants for a large enough $C_{0}$. Let $Y_{0}=\left(H_{0}, v_{0}, 0,0\right) \in \mathcal{Y}$ and $Y_{1}=\left(H_{1}, v_{1}, s_{1}, D_{1}\right) \in \mathcal{Y}$ be such that

$$
\left|H_{0}-1\right|_{1}+\left|v_{0}\right|_{1} \leqslant \eta,\left|H_{1}-1\right|_{1}+\left|v_{1}\right|_{1} \leqslant \eta,\left|s_{1}\right|+\left|D_{1}\right| \leqslant \eta .
$$

Let

$$
\begin{gathered}
\gamma:=\operatorname{Max}\left(\sqrt{\frac{2 C_{5}}{\mu}} \sqrt{\left|H_{0}-1\right|_{1}+\left|v_{0}\right|_{1}}, \sqrt{\frac{2 C_{6}}{\mu}} \sqrt{\left|H_{1}-1\right|_{1}+\left|v_{1}\right|_{1}},\left|s_{1}\right|+\left|D_{1}\right|\right), \\
\bar{s}:=\frac{\epsilon}{\gamma} s_{1}, \bar{D}:=\frac{\epsilon^{2}}{\gamma}\left(D_{1}-\frac{s_{1}}{2}\right),
\end{gathered}
$$


so that, thanks to $(2.34)$,

$$
s_{\bar{s}, \bar{D}}^{\epsilon, \gamma}=s_{1}, D_{\bar{s}, \bar{D}}^{\epsilon, \gamma}=D_{1}
$$

Note that, by $(2.41,2.45,2.46)$ and $(2.47)$,

$$
(\bar{s}, \bar{D}) \in \mathbb{D}
$$

By $(2.44,2.45)$ and $(2.46)$, we obtain that

$$
\gamma \in\left[0, \operatorname{Min}\left(\frac{\epsilon}{C_{2}}, \frac{\epsilon}{C_{3}}\right)\right]
$$

Then, by Proposition 8 , equations $(2.44,2.45)$ and $(2.50)$, there exists a function $Y^{0}=\left(H^{0}, v^{0}, s^{0}, D^{0}\right)$ : $[0,1 / \epsilon] \rightarrow \mathcal{Y}$ such that

$$
\begin{gathered}
\left(Y^{0}, u_{\bar{s}, \bar{D}}^{\epsilon, \gamma}-H_{0 x}(0)\right) \text { is a trajectory of the control system } \Sigma \text { on }[0,1 / \epsilon] \\
\qquad Y^{0}(0)=Y_{0} \\
\left|Y^{0}(t)-\tilde{Y}_{\bar{s}, \bar{D}}^{\epsilon, \gamma}(t)\right| \leqslant C_{5}\left|Y_{0}-Y_{e}\right| \forall t \in[0,1 / \epsilon] .
\end{gathered}
$$

By (2.46) and (2.53),

$$
\left|Y^{0}\left(\frac{1}{\epsilon}\right)-\tilde{Y}_{\bar{s}, \bar{D}}^{\epsilon, \gamma}\left(\frac{1}{\epsilon}\right)\right| \leqslant \frac{\mu \gamma^{2}}{2} .
$$

By Proposition 6, equations (2.41) and (2.50),

$$
\left|\tilde{Y}_{\bar{s}, \bar{D}}^{\epsilon, \gamma}\left(\frac{1}{\epsilon}\right)-Y^{\gamma, a_{\epsilon, \gamma}, b_{\epsilon, \gamma}^{\bar{s}, \bar{D}}(0)}\right| \leqslant C_{2} \epsilon \gamma^{2} \leqslant \frac{\mu \gamma^{2}}{2},
$$

which, with (2.54), gives

$$
\left|Y^{0}\left(\frac{1}{\epsilon}\right)-Y^{\gamma, a_{\epsilon, \gamma}, b_{\epsilon, \gamma}^{\bar{s}, \bar{D}}}\right| \leqslant \mu \gamma^{2}
$$

Similarly, by Propositions 7 and 9 , equations $(2.41,2.43-2.46,2.48)$ and $(2.50)$, there exists $Y^{1}=\left(H^{1}, v^{1}, s^{1}, D^{1}\right)$ : $\left[(1 / \epsilon)+T^{*}, T\right] \rightarrow \mathcal{Y}$ such that

$$
\begin{gathered}
\left(Y^{1}, u_{\bar{s}, \bar{D}}^{\epsilon, \gamma}-H_{1 x}(0)\right) \text { is a trajectory of the control system } \Sigma \text { on }\left[(1 / \epsilon)+T^{*}, T\right], \\
\qquad Y^{1}(T)=Y_{1}, \\
\left|Y^{1}(t)-\tilde{Y}_{\bar{s}, \bar{D}}^{\epsilon, \gamma}(t)\right| \leqslant C_{6}\left|Y_{1}-\left(1,0, s_{1}, D_{1}\right)\right| \forall t \in\left[(1 / \epsilon)+T^{*}, T\right] \\
\left|Y^{1}\left(\frac{1}{\epsilon}+T^{*}\right)-Y^{\gamma, a_{\epsilon, \gamma}, b_{\epsilon, \gamma}^{s, \bar{D}}}\left(T^{*}\right)\right| \leqslant \mu \gamma^{2} .
\end{gathered}
$$

By $(2.44,2.45)$ and $(2.46)$

$$
\gamma \leqslant \gamma_{0}
$$


From Proposition 5 , equations $(2.55,2.59)$ and $(2.60)$, there exists a trajectory $\left(Y^{*}, u^{*}\right):\left[0, T^{*}\right] \rightarrow \mathcal{Y}$ of the control system $\Sigma$ satisfying

$$
\begin{aligned}
& Y^{*}(0)=Y^{0}\left(\frac{1}{\epsilon}\right), \\
& \left|Y^{*}(t)-Y^{\gamma, a_{\epsilon, \gamma}, b_{\epsilon, \gamma}^{\bar{s}, \bar{\nu}}}(t)\right| \leqslant C_{1} \mu \gamma \forall t \in\left[0, T^{*}\right] \text {, } \\
& Y^{*}\left(T^{*}\right)=Y^{1}\left(\frac{1}{\epsilon}+T^{*}\right) .
\end{aligned}
$$

The map $(Y, u):[0, T] \rightarrow \mathcal{Y}$ defined by

$$
\begin{gathered}
(Y(t), u(t))=\left(Y^{0}(t), u_{\bar{s}, \bar{D}}^{\epsilon, \gamma}(t)-H_{0 x}(0)\right) \forall t \in[0,1 / \epsilon], \\
(Y(t), u(t))=\left(Y^{*}(t-(1 / \epsilon)), u^{*}(t-(1 / \epsilon))\right) \forall t \in\left[1 / \epsilon,(1 / \epsilon)+T^{*}\right], \\
(Y(t), u(t))=\left(Y^{1}(t), u_{\bar{s}, \bar{D}}^{\epsilon, \gamma}(t)-H_{1 x}(0)\right) \forall t \in\left[(1 / \epsilon)+T^{*}, T\right],
\end{gathered}
$$

is a trajectory of the control system $\Sigma$ which, by (2.52) and (2.57), satisfies (1.11). Finally the existence of $C_{0}>0$ such that $(1.12)$ holds follows from the construction of $(Y, u)$, equations $(1.7,2.15,2.27)$ to $(2.30,2.37$, $2.39,2.45,2.46,2.53,2.58)$ and $(2.62)$.

It remains to prove Proposition 5 and Proposition 6. We do it in Section 4 and Section 3 respectively.

\section{Proof of Proposition 6}

In this section we prove Proposition 6 . Let $(\bar{s}, \bar{D}) \in \mathbb{D}$. Let $\tilde{Y}_{\bar{s}, \bar{D}}^{\epsilon, \gamma}=\left(\tilde{H}_{\bar{s}, \bar{D}}^{\epsilon, \gamma}, \tilde{v}_{\bar{s}, \bar{D}}^{\epsilon, \gamma}, \tilde{s}_{\bar{s}, \bar{D}}^{\epsilon, \gamma}, \tilde{D}_{\bar{s}, \bar{D}}^{\epsilon, \gamma}\right) \in \mathcal{C}^{0}\left(\tilde{I}_{\bar{s}, \bar{D}}^{\epsilon, \gamma} ; \mathcal{Y}\right)$ be the (maximal) solution of

$$
\begin{gathered}
\left(\tilde{H}_{\bar{s}, \bar{D}}^{\epsilon, \gamma}\right)_{t}+\left(\tilde{H}_{\bar{s}, \bar{D}}^{\epsilon, \gamma} \tilde{v}_{\bar{s}, \bar{D}}^{\epsilon, \gamma}\right)_{x}=0 \\
\left(\tilde{v}_{\bar{s}, \bar{D}}^{\epsilon, \gamma}\right)_{t}(t, x)+\left(\tilde{H}_{\bar{s}, \bar{D}}^{\epsilon, \gamma}+\frac{\left(\tilde{v}_{\bar{s}, \bar{D}}^{\epsilon, \gamma}\right)^{2}}{2}\right)_{x}(t, x)=-u_{\bar{s}, \bar{D}}^{\epsilon, \gamma}(t) \\
\tilde{v}_{\bar{s}, \bar{D}}^{\epsilon, \gamma}(t, 0)=\tilde{v}_{\bar{s}, \bar{D}}^{\epsilon, \gamma}(t, 1)=0 \\
\tilde{H}_{\bar{s}, \bar{D}}^{\epsilon, \gamma}(0, x)=1, \tilde{v}_{\bar{s}, \bar{D}}^{\epsilon, \gamma}(0, x)=0 \\
\frac{\mathrm{d} \tilde{s}_{\bar{s}, \bar{D}}^{\epsilon, \gamma}}{\mathrm{d} t}(t)=u_{\bar{s}, \bar{D}}^{\epsilon, \gamma}(t) \\
\frac{\mathrm{d} \tilde{D}_{\bar{s}, \bar{D}}^{\epsilon, \gamma}}{\mathrm{d} t}(t)=\tilde{s}_{\bar{s}, \bar{D}}^{\epsilon, \gamma}(t) \\
\tilde{s}_{\bar{s}, \bar{D}}^{\epsilon, \gamma}(0)=0, \tilde{D}_{\bar{s}, \bar{D}}^{\epsilon, \gamma}(0)=0 .
\end{gathered}
$$

We consider solutions for positive time, so $\tilde{I}_{\bar{s}, \bar{D}}^{\epsilon, \gamma}$ is a subinterval of $[0,1 / \epsilon]$ containing 0 . The system $(1.1,1.2)$ is strictly hyperbolic if $H>0$ and the boundary condition (1.3) satisfies the Lopatinski condition (see e.g. [23], Vol. II, pp. 235-236) if $v^{2}<H$ for $x=0$ and $x=1$. Let $C_{7}>0$ be such that

$$
\left(|H-1|+|v| \leqslant 1 / C_{7}\right) \Rightarrow\left(v^{2}<H\right) .
$$

For $w \in \mathcal{C}^{0}(K)$, where $K$ is a compact subset of $\mathbb{R}^{n}$, let

$$
|w|_{0}:=\operatorname{Max}\{|w(x)| ; x \in K\} .
$$


For $w=\left(w_{1}, \ldots, w_{m}\right) \in \mathcal{C}^{0}\left(K ; \mathbb{R}^{m}\right)$, where $K$ is a compact subset of $\mathbb{R}^{n}$, let

$$
|w|_{0}:=\sum_{i=1}^{i=m}\left|w_{i}\right|_{0} .
$$

Then [19] (Th. 4.2, pp. 96) gives

$$
\left(\left|\tilde{H}_{\bar{s}, \bar{D}}^{\epsilon, \gamma}(t)-1\right|_{0}+\left|\tilde{v}_{\bar{s}, \bar{D}}^{\epsilon, \gamma}(t)\right|_{0} \leqslant 1 / C_{7} \forall t \in \tilde{I}_{\bar{s}, \bar{D}}^{\epsilon, \gamma}\right) \Rightarrow \tilde{I}_{\bar{s}, \bar{D}}^{\epsilon, \gamma}=[0,1 / \epsilon]
$$

(Moreover the left hand side of (3.9) for one solution implies the uniqueness of the solutions.) So, in order to prove Proposition 6 , it suffices to prove the existence of $C_{8}>2$ such that, for every $(\bar{s}, \bar{D}) \in \mathbb{D}$, for every $\epsilon \in\left(0,1 / C_{8}\right)$, and for every $\gamma \in\left[0, \epsilon / C_{8}\right]$, we have

$$
\left|\tilde{Y}_{\bar{s}, \bar{D}}^{\epsilon, \gamma}(t)-Y_{\bar{s}, \bar{D}}^{\epsilon, \gamma}(t)\right| \leqslant C_{8} \epsilon \gamma^{2} \forall t \in \tilde{I}_{\bar{s}, \bar{D}}^{\epsilon, \gamma}
$$

By construction,

$$
\tilde{s}_{\bar{s}, \bar{D}}^{\epsilon, \gamma}=s_{\bar{s}, \bar{D}}^{\epsilon, \gamma}, \tilde{D}_{\bar{s}, \bar{D}}^{\epsilon, \gamma}=D_{\bar{s}, \bar{D}}^{\epsilon, \gamma}
$$

In order to simplify the notations, we omit the indices $\bar{s}, \bar{D}, \epsilon, \gamma$. (We write $H$ instead of $H_{\bar{s}, \bar{D}}^{\epsilon, \gamma} \tilde{H}$ instead of $\tilde{H}_{\bar{s}, \bar{D}}^{\epsilon, \gamma}$ and so on.) Let us first put the hyperbolic system $(3.1,3.2)$ in a characteristic form by using the Riemann invariants. Let us recall that the Riemann invariants of the hyperbolic system $(2.54,2.55)$ are $-v+2 \sqrt{H}$, with the speed of propagation $v-\sqrt{H}$, and $v+2 \sqrt{H}$, with the speed of propagation $v+\sqrt{H}$ (see e.g. [7], pp. 146). Throughout this section only, let

$$
\begin{aligned}
R^{1} & :=-\tilde{v}+2 \sqrt{\tilde{H}}+v-2 \sqrt{1+h}-h v, \\
R^{2} & :=\tilde{v}+2 \sqrt{\tilde{H}}-v-2 \sqrt{1+h}+h v, \\
\lambda_{1} & :=-\frac{3 R^{1}}{4}+\frac{R^{2}}{4}+v-\sqrt{1+h}-h v, \\
\lambda_{2} & :=-\frac{R^{1}}{4}+\frac{3 R^{2}}{4}+v+\sqrt{1+h}-h v,
\end{aligned}
$$

with

$$
h:=H-1 .
$$

Then, equations (3.1) to (3.4) are equivalent, at least if the $L^{\infty}$ norm of $h, R^{1}$ and $R^{2}$ are small enough, to

$$
\begin{gathered}
R_{t}^{1}+\lambda_{1} R_{x}^{1}=A_{11} R^{1}+A_{12} R^{2}+G^{1}, \\
R_{t}^{2}+\lambda_{2} R_{x}^{2}=A_{21} R^{1}+A_{22} R^{2}+G^{2}, \\
R^{1}(t, 0)=R^{2}(t, 0), R^{1}(t, 1)=R^{2}(t, 1) \forall t \in \tilde{I}, \\
R^{1}(0, x)=R^{2}(0, x)=0 \forall x \in[0,1],
\end{gathered}
$$


with

$$
\begin{gathered}
A_{11}:=-\frac{3}{4}\left(v_{0 x}-\frac{h_{0 x}}{\sqrt{1+h}}-(h v)_{x}\right) \\
A_{12}:=\frac{1}{4}\left(v_{0 x}-\frac{h_{0 x}}{\sqrt{1+h}}-(h v)_{x}\right) \\
A_{21}:=\frac{1}{4}\left(v_{0 x}+\frac{h_{0 x}}{\sqrt{1+h}}-(h v)_{x}\right) \\
A_{22}:=-\frac{3}{4}\left(v_{0 x}+\frac{h_{0 x}}{\sqrt{1+h}}-(h v)_{x}\right) \\
G^{1}:=-(h v)_{t}+v_{0 x}\left(v-2 h v+\frac{h^{2}}{\sqrt{1+h}}+h^{2} v\right)+h_{0 x}\left(-v^{2}+\frac{2 h v}{\sqrt{1+h}}+h v^{2}\right) \\
G^{2}:=(h v)_{t}+v_{0 x}\left(-v+2 h v+\frac{h^{2}}{\sqrt{1+h}}-h^{2} v\right)+h_{0 x}\left(v^{2}+\frac{2 h v}{\sqrt{1+h}}-h v^{2}\right)
\end{gathered}
$$

Our first step is an estimate on $\left|R^{1}(t)\right|_{0}+\left|R^{2}(t)\right|_{0}$, which is given in the following lemma:

Lemma 10. There exists of $C_{9}>2$ such that, for every $(\bar{s}, \bar{D}) \in \mathbb{D}$, for every $\epsilon \in\left(0,1 / C_{9}\right]$, for every $\gamma \in$ $\left[0, \epsilon / C_{9}\right]$ and for every $t \in \tilde{I}$,

$$
\left|R^{1}(t)\right|_{0} \leqslant C_{9} \epsilon \gamma^{2},\left|R^{2}(t)\right|_{0} \leqslant C_{9} \epsilon \gamma^{2}
$$

Proof. Let us first point out that from (2.27) and (2.28), one gets the existence of $C_{10}>0$ such that, for every $(\bar{s}, \bar{D}) \in \mathbb{D}$, for every $\epsilon \in(0,1 / 2]$ for every $\gamma \in[0,+\infty)$ and for every $t \in[0,1 / \epsilon]$,

$$
\begin{aligned}
|v(t)|_{1} & \leqslant C_{10} \epsilon \gamma, \\
\left|v_{0 t}(t)\right|_{0} & \leqslant C_{10} \epsilon^{2} \gamma, \\
|h(t)|_{1} & \leqslant C_{10} \gamma, \\
\left|h_{0 t}(t)\right|_{0} & \leqslant C_{10} \epsilon \gamma .
\end{aligned}
$$

Using these inequalities one gets the existence of $C_{11}>0$ such that, for every $(\bar{s}, \bar{D}) \in \mathbb{D}$, for every $\epsilon \in\left(0,1 / C_{11}\right]$ for every $\gamma \in[0, \epsilon]$ and for every $t \in[0,1 / \epsilon]$,

$$
\begin{gathered}
\left|G^{1}(t)\right|_{0} \leqslant C_{11} \epsilon^{2} \gamma^{2},\left|G^{2}(t)\right|_{0} \leqslant C_{11} \epsilon^{2} \gamma^{2}, \\
\left|A_{11}(t)\right|_{0}+\left|A_{12}(t)\right|_{0} \leqslant C_{11} \gamma,\left|A_{21}(t)\right|_{0}+\left|A_{22}(t)\right|_{0} \leqslant C_{11} \gamma
\end{gathered}
$$


Let us now prove the following lemma:

Lemma 11. Let $\epsilon>0, T \in[0,1 / \epsilon], \lambda_{1}$ and $\lambda_{2}$ in $\mathcal{C}^{1}([0, T] \times[0,1]), r^{1}, r^{2}, f^{1}, f^{2}$ in $\mathcal{C}^{0}([0, T] \times[0,1])$ be such that

$$
\begin{gathered}
\lambda_{1}(t, x)<0 \forall(t, x) \in[0, T] \times[0,1], \\
\lambda_{2}(t, x)>0 \forall(t, x) \in[0, T] \times[0,1], \\
r_{t}^{1}+\lambda_{1} r_{x}^{1}=f^{1}, \\
r_{t}^{2}+\lambda_{2} r_{x}^{2}=f^{2}, \\
r^{1}(t, 0)=r^{2}(t, 0), r^{1}(t, 1)=r^{2}(t, 1) \forall t \in[0, T], \\
r^{1}(0, x)=r^{2}(0, x)=0 \forall x \in[0,1], \\
\left|f^{1}(t)\right|_{0} \leqslant \epsilon \text { and }\left|f^{2}(t)\right|_{0} \leqslant \epsilon \forall t \in[0, T] .
\end{gathered}
$$

Then, for every $t \in[0, T]$,

$$
\left|r^{1}(t)\right|_{0} \leqslant 1 \text { and }\left|r^{2}(t)\right|_{0} \leqslant 1
$$

Remark 12. Of course, with $r^{1}$ and $r^{2}$ just continuous, equations (3.30) and (3.31) have to be understood by using the corresponding system of integrals along the characteristics, that is, if $\xi_{1}\left(\operatorname{resp} . \xi_{2}\right):\left[t_{-}, t_{+}\right] \subset[0, T]$ $\rightarrow[0,1]$ is a solution of

$$
\begin{gathered}
\dot{x}=\lambda_{1}(t, x), \\
\left(\operatorname{resp} . \dot{x}=\lambda_{2}(t, x)\right),
\end{gathered}
$$

then

$$
\begin{gathered}
r_{1}\left(t, \xi_{1}(t)\right)=r_{1}\left(t_{-}, \xi_{1}\left(t_{-}\right)\right)+\int_{t_{-}}^{t} f_{1}\left(s, \xi_{1}(s)\right) \mathrm{d} s \forall t \in\left[t_{-}, t_{+}\right], \\
\left(\operatorname{resp.} r_{2}\left(t, \xi_{2}(t)\right)=r_{2}\left(t_{-}, \xi_{1}\left(t_{-}\right)\right)+\int_{t_{-}}^{t} f_{2}\left(s, \xi_{2}(s)\right) \mathrm{d} s \forall t \in\left[t_{-}, t_{+}\right]\right) .
\end{gathered}
$$

We use this convention until the end of this paper. To prove Lemma 10 from Lemma 11 one could assume in Lemma 11 that $r^{1}$ and $r^{2}$ are of class $\mathcal{C}^{1}$. But one needs to consider the case where $r^{1}$ and $r^{2}$ are just continuous for the proof of Lemma 14 .

The proof of Lemma 11 readily follows by looking at the evolution of $r^{1}$ and $r^{2}$ along the characteristics (see (3.38) and (3.39)). Indeed, let $a \in(0,1)$ and $\tau \in[0, T]$. Let $\xi \in \mathcal{C}^{0}([0, \tau] ;[0,1])$ be such that there exist $k \in \mathbb{N} \backslash\{0\}$ and $0=\tau_{k}<\tau_{k-1}<\ldots<\tau_{1}<\tau_{0}=\tau$ satisfying

$$
\begin{gathered}
\xi \in \mathcal{C}^{1}\left([0, \tau] \backslash\left\{\tau_{0}, \ldots, \tau_{k}\right\}\right), \\
\dot{\xi}=\lambda_{1}(t, \xi) \text { on }\left(\tau_{j}, \tau_{j-1}\right) \forall j \text { odd } \in\{1, \ldots, k\}, \\
\dot{\xi}=\lambda_{2}(t, \xi) \text { on }\left(\tau_{j}, \tau_{j-1}\right) \forall j \text { even } \in\{1, \ldots, k\} \\
\xi(\tau)=a .
\end{gathered}
$$

The existence (and uniqueness of such a $\xi$ ) follows from (3.28) and (3.29). Then, by $(3.32,3.33,3.38)$ and (3.39),

$$
r_{1}(\tau, a)=\sum_{j \text { odd } \in\{1, \ldots, k\}} \int_{\tau_{j}}^{\tau_{j-1}} f_{1}(s, \xi(s)) \mathrm{d} s+\sum_{j \text { even } \in\{1, \ldots, k\}} \int_{\tau_{j}}^{\tau_{j-1}} f_{2}(s, \xi(s)) \mathrm{d} s,
$$


which, with (3.34), gives

Similarly one gets

$$
\left|r^{1}(\tau, a)\right| \leqslant \epsilon \tau
$$

which ends the proof of Lemma 11.

$$
\left|r^{2}(\tau, a)\right| \leqslant \epsilon \tau,
$$

Let $C_{12}>2$ be such that, for every $(\bar{s}, \bar{D}) \in \mathbb{D}$, for every $\epsilon \in\left(0,1 / C_{12}\right]$, for every $\gamma \in\left[0,1 / C_{12}\right]$, for every $R^{1} \in\left[-1 / C_{12}, 1 / C_{12}\right]$ and for every $R^{2} \in\left[-1 / C_{12}, 1 / C_{12}\right], \lambda_{1}$ defined by (3.14) and $\lambda_{2}$ defined by (3.15) satisfy

$$
\lambda_{1}<0<\lambda_{2}
$$

Let $C_{13}$ and $C_{9}$ be such that

$$
\begin{aligned}
C_{13}:= & \operatorname{Max}\left(C_{12}, 2 C_{11}\right), \\
& C_{9}>C_{13} .
\end{aligned}
$$

We claim that, with such a $C_{9}$, Lemma 10 holds. Indeed, if this is not the case, there exist $\epsilon \in\left(0,1 / C_{9}\right]$, $\gamma \in\left(0, \epsilon / C_{9}\right]$ and $T \in \tilde{I}$ satisfying

$$
\begin{gathered}
\left|R^{1}(t)\right|_{0} \leqslant C_{9} \epsilon \gamma^{2},\left|R^{2}(t)\right|_{0} \leqslant C_{9} \epsilon \gamma^{2} \forall t \in[0, T], \\
\left|R^{1}(T)\right|_{0}=C_{9} \epsilon \gamma^{2} \text { or }\left|R^{2}(T)\right|_{0}=C_{9} \epsilon \gamma^{2}
\end{gathered}
$$

From $(3.26,3.27)$ and $(3.43)$, one gets

$$
\left|\left(A_{11} R^{1}+A_{12} R^{2}+G^{1}\right)(t)\right|_{0} \leqslant C_{11}\left(C_{9} \frac{\gamma}{\epsilon}+1\right) \epsilon^{2} \gamma^{2} \leqslant 2 C_{11} \epsilon^{2} \gamma^{2} \forall t \in[0, T] .
$$

Similarly

$$
\left|\left(A_{21} R^{1}+A_{22} R^{2}+G^{1}\right)(t)\right|_{0} \leqslant 2 C_{11} \epsilon^{2} \gamma^{2} \forall t \in[0, T] .
$$

From $(3.41,3.42)$ and (3.43),

$$
\left|R^{1}(t)\right|_{0} \leqslant \frac{1}{C_{9}^{4}} \leqslant \frac{1}{C_{12}} \forall t \in[0, T]
$$

Similarly

$$
\left|R^{2}(t)\right|_{0} \leqslant \frac{1}{C_{12}} \forall t \in[0, T]
$$

From the definition of $C_{12}$, equations $(3.41,3.42,3.47)$ and (3.48), one gets

$$
\lambda_{1}<0<\lambda_{2} \text { on }[0, T] \times[0,1] .
$$

From Lemma 11, equations (3.17) to $(3.20,3.41,3.45,3.46)$ and $(3.49)$, we have

$$
\left|R^{1}(t)\right|_{0} \leqslant 2 C_{11} \epsilon \gamma^{2} \leqslant C_{13} \epsilon \gamma^{2} \forall t \in[0, T] .
$$

Similarly

$$
\left|R^{2}(t)\right|_{0} \leqslant C_{13} \epsilon \gamma^{2} \forall t \in[0, T] .
$$

But $(3.42,3.44,3.50)$ and $(3.51)$ are in contradiction. This ends the proof of Lemma 10. 
Remark 13. The term $h v$ in the definition (3.12) to (3.15) of $R^{1}, R^{2}, \lambda_{1}$ and $\lambda_{2}$ is important even if it is of order $0\left(\epsilon \gamma^{2}\right)$. Indeed if one omits this term in (3.12) to (3.15), one gets, instead of (3.26), the weaker estimates

$$
\left|G^{1}(t)\right|_{0} \leqslant C_{11} \epsilon \gamma^{2},\left|G^{2}(t)\right|_{0} \leqslant C_{11} \epsilon \gamma^{2}
$$

which are not enough to get Lemma 10.

In view of (3.17) and (3.18), in order to have $\mathcal{C}^{0}$-estimates on $R_{x}^{1}(t)$ and $R_{x}^{2}(t)$, it suffices to have $\mathcal{C}^{0}$-estimates on $\bar{R}^{1}(t):=R_{t}^{1}(t)$ and $\bar{R}^{2}(t):=R_{t}^{2}(t)$. Taking the derivative with respect to $t$ of $(3.17,3.18)$ and $(3.19)$, one gets, using $(3.14,3.15,3.17)$ and $(3.18)$ to eliminate $R_{x}^{1}$ and $R_{x}^{2}$,

$$
\begin{gathered}
\bar{R}_{t}^{1}+\lambda_{1} \bar{R}_{x}^{1}=-\frac{3}{4 \lambda_{1}}\left(\bar{R}^{1}\right)^{2}+\frac{1}{4 \lambda_{1}} \bar{R}^{1} \bar{R}^{2}+\bar{A}_{11} \bar{R}^{1}+\bar{A}_{12} \bar{R}^{2}+\bar{G}^{1} \\
\bar{R}_{t}^{2}+\lambda_{2} \bar{R}_{x}^{2}=\frac{3}{4 \lambda_{2}}\left(\bar{R}^{2}\right)^{2}-\frac{1}{4 \lambda_{1}} \bar{R}^{1} \bar{R}^{2}+\bar{A}_{21} \bar{R}^{1}+\bar{A}_{22} \bar{R}^{2}+\bar{G}^{2} \\
\bar{R}^{1}(t, 0)=\bar{R}^{2}(t, 0), \bar{R}^{1}(t, 1)=\bar{R}^{2}(t, 1) \forall t \in \tilde{I}
\end{gathered}
$$

with

$$
\begin{gathered}
\bar{A}_{11}:=A_{11}+\frac{3}{4 \lambda_{1}}\left(A_{11} R^{1}+A_{12} R^{2}+G^{1}\right)+\frac{1}{\lambda_{1}}(v-\sqrt{1+h}-h v)_{t}, \\
\bar{A}_{12}:=A_{12}-\frac{1}{4 \lambda_{1}}\left(A_{11} R^{1}+A_{12} R^{2}+G^{1}\right), \\
\bar{G}^{1}:=A_{11 t} R^{1}+A_{12 t} R^{2}+G_{t}^{1}-\frac{1}{\lambda_{1}}\left(A_{11} R^{1}+A_{12} R^{2}+G^{1}\right)(v-\sqrt{1+h}-h v)_{t}, \\
\bar{A}_{21}:=A_{21}+\frac{1}{4 \lambda_{2}}\left(A_{21} R^{1}+A_{22} R^{2}+G^{2}\right), \\
\bar{A}_{22}:=A_{22}-\frac{3}{4 \lambda_{2}}\left(A_{21} R^{1}+A_{22} R^{2}+G^{2}\right)+\frac{1}{\lambda_{2}}(v+\sqrt{1+h}-h v)_{t}, \\
\bar{G}^{2}:=A_{21 t} R^{1}+A_{22 t} R^{2}+G_{t}^{2}-\frac{1}{\lambda_{2}}\left(A_{21} R^{1}+A_{22} R^{2}+G^{2}\right)(v+\sqrt{1+h}-h v)_{t} .
\end{gathered}
$$

From $(3.17,3.18)$ and $(3.20)$, one gets

$$
\bar{R}^{1}(0, x)=\bar{R}^{2}(0, x) \forall x \in[0,1]
$$

Let us first point out that from (2.27) and (2.28), one gets the existence of $C_{14}>0$ such that, for every $(\bar{s}, \bar{D}) \in \mathbb{D}$, for every $\epsilon \in(0,1 / 2]$ for every $\gamma \in[0,+\infty)$ and for every $t \in[0,1 / \epsilon]$,

$$
\begin{aligned}
\left|v_{0 t}(t)\right|_{1} & \leqslant C_{14} \epsilon^{2} \gamma \\
\left|v_{0 t t}(t)\right|_{0} & \leqslant C_{14} \epsilon^{3} \gamma \\
\left|h_{0 t}(t)\right|_{1} & \leqslant C_{14} \epsilon \gamma \\
\left|h_{0 t t}(t)\right|_{0} & \leqslant C_{14} \epsilon^{2} \gamma .
\end{aligned}
$$

Hence, using also $(3.22,3.24)$ and Lemma 10 , one gets the existence of $C_{15}>0$ such that, for every $(\bar{s}, \bar{D}) \in \mathbb{D}$, for every $\epsilon \in\left(0,1 / C_{15}\right]$ for every $\gamma \in\left[0, \epsilon / C_{15}\right]$ and for every $t \in \tilde{I}$,

$$
\begin{gathered}
\left|\bar{G}^{1}(t)\right|_{0} \leqslant C_{15} \epsilon^{3} \gamma^{2},\left|\bar{G}^{2}(t)\right|_{0} \leqslant C_{15} \epsilon^{3} \gamma^{2} \\
\left|\bar{A}_{11}(t)\right|_{0}+\left|\bar{A}_{12}(t)\right|_{0} \leqslant C_{15} \gamma,\left|\bar{A}_{21}(t)\right|_{0}+\left|\bar{A}_{22}(t)\right|_{0} \leqslant C_{15} \gamma
\end{gathered}
$$


Again, proceeding as in the proof of Lemma 10 (one easily sees that the quadratic terms in $\bar{R}^{1}, \bar{R}^{2}$ in the right hand sides of (3.52) and (3.53) do not create any problem), one gets the following lemma:

Lemma 14. There exists $C_{16}>0$ such that, for every $(\bar{s}, \bar{D}) \in \mathbb{D}$, for every $\epsilon \in\left(0,1 / C_{16}\right]$ for every $\gamma \in$ $\left[0, \epsilon / C_{16}\right]$ and for every $t \in \tilde{I}$,

$$
\left|\bar{R}^{1}(t)\right|_{0} \leqslant C_{16} \epsilon^{2} \gamma^{2},\left|\bar{R}^{2}(t)\right|_{0} \leqslant C_{16} \epsilon^{2} \gamma^{2},
$$

This lemma, together with $(3.14,3.15,3.17,3.18)$, Lemma 10, equations $(3.26)$ and (3.27) gives:

Lemma 15. There exists $C_{17}>0$ such that, for every $(\bar{s}, \bar{D}) \in \mathbb{D}$, for every $\epsilon \in\left(0,1 / C_{17}\right]$ for every $\gamma \in$ $\left[0, \epsilon / C_{17}\right]$ and for every $t \in \tilde{I}$,

$$
\left|R^{1}(t)\right|_{1} \leqslant C_{17} \epsilon \gamma^{2},\left|R^{2}(t)\right|_{1} \leqslant C_{17} \epsilon \gamma^{2}
$$

But, by (3.12) and (3.13),

$$
\begin{gathered}
\tilde{v}-v=\frac{R_{2}-R_{1}}{2}-h v, \\
\tilde{H}-H=\left(\frac{R_{1}+R_{2}}{4}\right)^{2}+\frac{\sqrt{1+h}}{2}\left(R_{1}+R_{2}\right) .
\end{gathered}
$$

Hence, using Lemma 15 , equations $(3.11,3.22,3.24)$ one gets $(3.10)$ for every $(\bar{s}, \bar{D}) \in \mathbb{D}$, for every $\epsilon \in\left(0,1 / C_{8}\right]$, for every $\gamma \in\left[0, \epsilon / C_{8}\right]$ and for every $t \in \tilde{I}$ provided that $C_{8}$ is large enough. This ends the proof of Proposition 6 .

\section{Proof of Proposition 5}

In this section, we prove Proposition 5. Since $(Y, u)=((H, v, s, D), u)$ is a trajectory of the control system $\Sigma$ if and only if $((H, v, s-a, D-a t-b), u)$ is a trajectory of the control system $\Sigma$, we may assume without loss of generality that $a=b=0$. For simplicity we also omit the indices $a(=0)$ and $b(=0)$. Let us first put the hyperbolic system $(1.1,1.2)$ in a characteristic form by letting

$$
\begin{aligned}
& R^{1}:=-v+2 \sqrt{H}-2 \sqrt{H^{\gamma}}-\sigma, \\
& R^{2}:=v+2 \sqrt{H}-2 \sqrt{H^{\gamma}}+\sigma, \\
& \lambda_{1}:=-\frac{3 R^{1}}{4}+\frac{R^{2}}{4}-\sqrt{H^{\gamma}}-\sigma, \\
& \lambda_{2}:=-\frac{R^{1}}{4}+\frac{3 R^{2}}{4}+\sqrt{H^{\gamma}}-\sigma,
\end{aligned}
$$

with

$$
\sigma:=s-\gamma t
$$

Then, at least if $\gamma$ and the $L^{\infty}$-norm of $R^{1}$ and $R^{2}$ are small enough, equations (1.1) to (1.5) are equivalent to

$$
\begin{gathered}
R_{t}^{\mathrm{tr}}+\Lambda R_{x}^{\mathrm{tr}}=M_{1} R^{\mathrm{tr}}+\sigma M_{2}, \\
R^{2}(t, 0)-R^{1}(t, 0)=R^{2}(t, 1)-R^{1}(t, 1)=2 \sigma(t), \\
\frac{\mathrm{d} \sigma}{\mathrm{d} t}(t)=w(t), \\
\frac{\mathrm{d} \delta}{\mathrm{d} t}(t)=\sigma(t),
\end{gathered}
$$


with

$$
\begin{aligned}
& w:=u-\gamma, \delta:=D-\frac{\gamma t^{2}}{2}, \\
& R:=\left(R^{1}, R^{2}\right), \Lambda:=\left(\begin{array}{cc}
\lambda_{1} & 0 \\
0 & \lambda_{2}
\end{array}\right), \\
& M_{1}:=\left(\begin{array}{cc}
-\frac{3 \gamma}{4 \sqrt{H^{\gamma}}} & \frac{\gamma}{4 \sqrt{H^{\gamma}}} \\
-\frac{\gamma}{4 \sqrt{H^{\gamma}}} & \frac{3 \gamma}{4 \sqrt{H^{\gamma}}}
\end{array}\right), M_{2}:=\left(\begin{array}{c}
-\frac{\gamma}{\sqrt{H^{\gamma}}} \\
-\frac{{ }^{H \gamma}}{\sqrt{H^{\gamma}}}
\end{array}\right),
\end{aligned}
$$

and where, for a matrix $M, M^{\text {tr }}$ denotes the transpose of $M$. Note that (1.10) is equivalent to

$$
\int_{0}^{1}\left(R^{1}+R^{2}\right) \sqrt{H^{\gamma}}+\frac{\left(R^{1}+R^{2}\right)^{2}}{18} \mathrm{~d} x=0 .
$$

Let $Z=\left(Z^{1}, Z^{2}\right)$ be defined by

$$
Z^{\mathrm{tr}}:=M_{3} R_{x}^{\mathrm{tr}}-M_{4} R^{\mathrm{tr}}
$$

with

$$
M_{3}:=\left(\begin{array}{cc}
1-\frac{5 \gamma}{4} x & 0 \\
0 & -1+\frac{5 \gamma}{4} x
\end{array}\right), M_{4}:=\left(\begin{array}{cc}
\frac{5 \gamma}{8} & -\frac{\gamma}{8} \\
\frac{\gamma}{8} & -\frac{5 \gamma}{8}
\end{array}\right)
$$

From $(4.6,4.11,4.12)$ and $(4.14)$ to $(4.15)$, one gets

$$
Z_{t}^{\operatorname{tr}}+\Lambda Z_{x}^{\operatorname{tr}}=F
$$

with $F=F\left(x, \gamma, \sigma, R, R_{x}\right)$ defined by

$$
\begin{aligned}
F:= & \left(F_{1}, F_{2}\right)^{\mathrm{tr}}:=\left(M_{3} M_{1}-M_{3} \Lambda_{x}+\Lambda M_{3 x}+M_{4} \Lambda-\Lambda M_{4}\right) R_{x}^{\mathrm{tr}} \\
& +\left(M_{3} M_{1 x}-M_{4} M_{1}\right) R^{\mathrm{tr}}+\sigma\left(M_{3} M_{2 x}-M_{4} M_{2}\right) .
\end{aligned}
$$

From $(4.6)$ to $(4.8,4.11,4.12)$ and $(4.14)$ to $(4.15)$, one gets

$$
2 w(t)=\left(1+\frac{\gamma}{4}\right)\left(Z^{2}(t, 0)-Z^{1}(t, 0)\right)+B_{0}=(1+\gamma)\left(Z^{2}(t, 1)-Z^{1}(t, 1)\right)+B_{1},
$$

with $B_{0}=B_{0}\left(\gamma, R(t, 0), R_{x}(t, 0)\right)$ defined by

$$
\begin{aligned}
B_{0}:= & \left(-\frac{R^{1}(t, 0)+R^{2}(t, 0)}{4}+\frac{\gamma^{2}}{16+4 \gamma+16 \sqrt{1+\frac{\gamma}{2}}}\right)\left(R_{x}^{1}(t, 0)+R_{x}^{2}(t, 0)\right) \\
& +\frac{\gamma}{2}\left(\frac{1}{\sqrt{1+\frac{\gamma}{2}}}-1-\frac{\gamma}{4}\right)\left(R^{1}(t, 0)+R^{2}(t, 0)\right)
\end{aligned}
$$


and $B_{1}=B_{1}\left(\gamma, R(t, 1), R_{x}(t, 1)\right)$ defined by

$$
\begin{aligned}
B_{1}:= & \left(-\frac{R^{1}(t, 1)+R^{2}(t, 1)}{4}+\frac{\gamma^{2}}{16-4 \gamma+16 \sqrt{1-\frac{\gamma}{2}}}-\frac{5 \gamma^{2}}{4}\right)\left(R_{x}^{1}(t, 1)+R_{x}^{2}(t, 1)\right) \\
& +\frac{\gamma}{2}\left(\frac{1}{\sqrt{1-\frac{\gamma}{2}}}-1-\gamma\right)\left(R^{1}(t, 1)+R^{2}(t, 1)\right) .
\end{aligned}
$$

Note that, for some $C_{18}>0$, one has for every $\left(\gamma, r, r_{x}\right) \in \mathbb{R} \times \mathbb{R}^{2} \times \mathbb{R}^{2}$ with $|\gamma|+|\sigma|+|r|+\left|r_{x}\right| \leqslant 1 / 2$,

$$
\begin{gathered}
\left(\left|B_{0}\right|+\left|B_{1}\right|\right)\left(\gamma, r, r_{x}\right) \leqslant C_{18}\left(\gamma^{2}|r|+\gamma^{2}\left|r_{x}\right|+|r|\left|r_{x}\right|\right), \\
\left(\left|B_{0 r}\right|+\left|B_{1 r}\right|\right)\left(\gamma, r, r_{x}\right) \leqslant C_{18}\left(\gamma^{2}+\left|r_{x}\right|\right), \\
\left(\left|B_{0 r_{x}}\right|+\left|B_{1 r_{x}}\right|\right)\left(\gamma, r, r_{x}\right) \leqslant C_{18}\left(\gamma^{2}+|r|\right) .
\end{gathered}
$$

Similarly, using $(4.3,4.4,4.12,4.15,4.17)$ and straightforward computations, one gets the existence of $C_{19}>0$ such that, for every $\left(x, \gamma, \sigma, r, r_{x}\right) \in[0,1] \times \mathbb{R} \times \mathbb{R} \times \mathbb{R}^{2} \times \mathbb{R}^{2}$ with $|\gamma|+|\sigma|+|r|+\left|r_{x}\right| \leqslant 1 / 2$,

$$
\begin{gathered}
\left(|F|+\left|F_{x}\right|\right)\left(x, \gamma, \sigma, r, r_{x}\right) \leqslant C_{19}\left(\gamma^{2}\left(|\sigma|+|r|+\left|r_{x}\right|\right)+\left|r_{x}\right|\left(\gamma|\sigma|+\gamma|r|+\left|r_{x}\right|\right)\right) \\
\left|F_{\sigma}\right|\left(x, \gamma, \sigma, r, r_{x}\right) \leqslant C_{19}\left(\gamma^{2}+\gamma\left|r_{x}\right|\right) \\
\left|F_{r}\right|\left(x, \gamma, \sigma, r, r_{x}\right) \leqslant C_{19}\left(\gamma^{2}+\gamma\left|r_{x}\right|\right) \\
\left|F_{r_{x}}\right|\left(x, \gamma, \sigma, r, r_{x}\right) \leqslant C_{19}\left(\gamma^{2}+\left|r_{x}\right|+\gamma|\sigma|+\gamma|r|\right)
\end{gathered}
$$

Let us point out that one can recover $\sigma$ from $Z$. Indeed from (4.14) and (4.15), one gets

$$
Z^{1}+Z^{2}=\left(1-\frac{5 \gamma}{4} x\right)\left(R_{x}^{1}-R_{x}^{2}\right)-\frac{3 \gamma}{4}\left(R^{1}-R^{2}\right)
$$

Multiplying (4.28) by $(1-(5 \gamma x / 4))^{-2 / 5}$, integrating the obtained equality and using an integration by parts together with (4.7), one obtains

$$
\sigma(t)=\frac{1}{2\left(1-\left(1-\frac{5 \gamma}{4}\right)^{3 / 5}\right)} \int_{0}^{1} \frac{1}{\left(1-\frac{5 \gamma}{4} x\right)^{2 / 5}}\left(Z^{1}+Z^{2}\right)(t, x) \mathrm{d} x
$$

From (4.29) one easily sees than one can also recover $R$ from $Z$. More precisely, straightforward computations (together with (4.29)) give the following lemma, whose proof is omitted:

Lemma 16. There exists $\gamma_{5} \in(0,1 / 2]$ and $C_{20}>0$ such that, for every $\gamma \in\left[-\gamma_{5}, \gamma_{5}\right] \backslash\{0\}$, the two following properties hold.

(i) For every $Z=\left(Z^{1}, Z^{2}\right) \in \mathcal{C}^{0}([0,1])^{2}$ with

$$
\frac{1}{\gamma}\left|\int_{0}^{1} Z^{1}+Z^{2} d x\right|+|Z|_{0} \leqslant \gamma_{5}
$$


there exists one and only one $\left(\mathbf{R}^{\gamma}(Z), \mathbf{S}^{\gamma}(Z)\right) \in \mathcal{C}^{1}([0,1])^{2} \times \mathbb{R}$ such that, with $\mathbf{R}^{\gamma}(Z)=\left(R^{1}, R^{2}\right)$,

$$
\begin{gathered}
Z^{t r}=M_{3} R_{x}^{t r}-M_{4} R^{t r}, \\
R^{2}(0)-R^{1}(0)=R^{2}(1)-R^{1}(1)=2 \mathbf{S}^{\gamma}(Z), \\
\int_{0}^{1} \frac{R^{1}+R^{2}}{2} \sqrt{H^{\gamma}}+\frac{\left(R^{1}+R^{2}\right)^{2}}{16} \mathrm{~d} x=0 .
\end{gathered}
$$

One has

$$
\mathbf{S}^{\gamma}(Z)=\frac{1}{2\left(1-\left(1-\frac{5 \gamma}{4}\right)^{3 / 5}\right)} \int_{0}^{1} \frac{1}{\left(1-\frac{5 \gamma}{4} x\right)^{2 / 5}}\left(Z^{1}+Z^{2}\right)(x) \mathrm{d} x
$$

(ii) For every $Z=\left(Z^{1}, Z^{2}\right) \in \mathcal{C}^{0}([0,1])^{2}$ and for every $\bar{Z}=\left(\bar{Z}^{1}, \bar{Z}^{2}\right) \in \mathcal{C}^{0}([0,1])^{2}$ such that

$$
\frac{1}{\gamma}\left|\int_{0}^{1} Z^{1}+Z^{2} \mathrm{~d} x\right|+|Z|_{0} \leqslant \gamma_{5}, \frac{1}{\gamma}\left|\int_{0}^{1} Z^{1}+Z^{2} \mathrm{~d} x\right|+|Z|_{0} \leqslant \gamma_{5}
$$

one has

$$
\begin{gathered}
\left|\mathbf{R}^{\gamma}(Z)\right|_{0}+\left|\mathbf{S}^{\gamma}(Z)\right| \leqslant C_{20}\left(\frac{1}{\gamma}\left|\int_{0}^{1} Z^{1}+Z^{2} \mathrm{~d} x\right|+|Z|_{0}\right), \\
\left|\mathbf{R}^{\gamma}(Z)_{x}\right|_{0} \leqslant C_{20}|Z|_{0}, \\
\left|\mathbf{R}^{\gamma}(\bar{Z})-\mathbf{R}^{\gamma}(Z)\right|_{0}+\left|\mathbf{S}^{\gamma}(\bar{Z})-\mathbf{S}^{\gamma}(Z)\right| \leqslant C_{20}\left(\frac{1}{\gamma}\left|\int_{0}^{1} \bar{Z}^{1}+\bar{Z}^{2}-Z^{1}-Z^{2} \mathrm{~d} x\right|+|\bar{Z}-Z|_{0}\right), \\
\left|\mathbf{R}^{\gamma}(\bar{Z})_{x}-\mathbf{R}^{\gamma}(Z)_{x}\right|_{0} \leqslant C_{20}|\bar{Z}-Z|_{0} .
\end{gathered}
$$

For $Z=\left(Z^{1}, Z^{2}\right) \in \mathcal{C}^{0}\left(\left[0, T^{*}\right] \times[0,1]\right)^{2}$ with

$$
\frac{1}{\gamma}\left|\int_{0}^{1}\left(Z^{1}+Z^{2}\right)(\cdot, x) \mathrm{d} x\right|_{0}+|Z|_{0} \leqslant \gamma_{5}
$$

and $\gamma \in\left[-\gamma_{5}, \gamma_{5}\right] \backslash\{0\}$ let us define $\mathbf{R}^{\gamma}(Z)$ in $\mathcal{C}^{0}\left(\left[0, T^{*}\right] ; \mathcal{C}^{1}\left(\left[0, T^{*}\right]\right)\right)^{2}$ and $\mathbf{S}^{\gamma}(Z)$ in $\mathcal{C}^{0}\left(\left[0, T^{*}\right]\right)$ by

$$
\mathbf{R}^{\gamma}(Z)(t, \cdot):=\mathbf{R}^{\gamma}(Z(t, \cdot)), \mathbf{S}^{\gamma}(Z)(t):=\mathbf{S}^{\gamma}(Z(t, \cdot)) .
$$

Let $\left(\lambda_{1}, \lambda_{2}\right)$ in $\mathcal{C}^{0}\left(\left[0, T^{*}\right] ; \mathcal{C}^{1}[0,1]\right)^{2}$ be such that

$$
\lambda_{1}(t, x)<0, \lambda_{2}(t, x)>0 \forall(t, x) \in\left[0, T^{*}\right] \times[0,1] .
$$


Let $\left(\tau_{0}, \tau_{1}^{\lambda}, \tau_{2}^{\lambda}, \tau_{3}^{\lambda}, \tau_{4}^{\lambda}\right) \in\left[0, T^{*}\right]^{5}$ be such that

$$
\begin{gathered}
\tau_{0}=\frac{T^{*}}{2}-1, \\
\left(\dot{\xi}=\lambda_{2}(t, \xi) \text { and } \xi\left(\tau_{0}\right)=0\right) \Rightarrow\left(\xi\left(\tau_{1}^{\lambda}\right)=1\right), \\
\left(\dot{\xi}=\lambda_{1}(t, \xi) \text { and } \xi\left(\tau_{1}^{\lambda}\right)=1\right) \Rightarrow\left(\xi\left(\tau_{2}^{\lambda}\right)=0\right), \\
\left(\dot{\xi}=\lambda_{1}(t, \xi) \text { and } \xi\left(\tau_{0}\right)=1\right) \Rightarrow\left(\xi\left(\tau_{3}^{\lambda}\right)=0\right), \\
\left(\dot{\xi}=\lambda_{2}(t, \xi) \text { and } \xi\left(\tau_{3}^{\lambda}\right)=0\right) \Rightarrow\left(\xi\left(\tau_{4}^{\lambda}\right)=1\right) .
\end{gathered}
$$

Note that, by (4.31), if such a $\left(\tau_{1}^{\lambda}, \tau_{2}^{\lambda}, \tau_{3}^{\lambda}, \tau_{4}^{\lambda}\right)$ exists, it is unique. Moreover $\left(\tau_{1}^{\lambda}, \tau_{2}^{\lambda}, \tau_{3}^{\lambda}, \tau_{4}^{\lambda}\right)$ indeed exists if

$$
\left|\lambda_{1}+1\right|_{\mathcal{C}^{0}\left(\left[0, T^{*}\right] ; \mathcal{C}^{1}[0,1]\right)}+\left|\lambda_{2}-1\right|_{\mathcal{C}^{0}\left(\left[0, T^{*}\right] ; \mathcal{C}^{1}[0,1]\right)}
$$

is small enough and the map

$$
\begin{array}{r}
\mathcal{C}^{0}\left(\left[0, T^{*}\right] ; \mathcal{C}^{1}[0,1]\right)^{2} \rightarrow \mathbb{R}^{4} \\
\lambda=\left(\lambda_{1}, \lambda_{2}\right) \mapsto\left(\tau_{1}^{\lambda}, \tau_{2}^{\lambda}, \tau_{3}^{\lambda}, \tau_{4}^{\lambda}\right)
\end{array}
$$

is of class $\mathcal{C}^{1}$ on an open neighborhood of $(-1,1) \in \mathcal{C}^{0}\left(\left[0, T^{*}\right] ; \mathcal{C}^{1}[0,1]\right)^{2}$ (for the usual topology on the Banach space $\left.\mathcal{C}^{0}\left(\left[0, T^{*}\right] ; \mathcal{C}^{1}[0,1]\right)^{2}\right)$. This can be proved by using classical estimates for ordinary differential equations (mainly Gronwall's lemma) together with the implicit function theorem. Let $\theta: \mathcal{C}^{0}\left(\left[0, T^{*}\right] ; \mathcal{C}^{1}[0,1]\right)^{2} \rightarrow \mathbb{R}^{2}$ be defined by

$$
\theta\left(\lambda_{1}, \lambda_{2}\right):=\left(\tau_{3}^{\lambda}-\tau_{1}^{\lambda}, \tau_{4}^{\lambda}-\tau_{2}^{\lambda}\right)
$$

One easily sees that $\left.\theta^{\prime}(-1,1)\left(\mathcal{C}^{0}\left(\left[0, T^{*}\right] ; \mathcal{C}^{1}[0,1]\right)^{2}\right)\right)=\mathbb{R}^{2}$ (by the way this property follows from (4.79) and $(4.80)$ below). Hence there exists a map $\Pi: \mathcal{C}^{0}\left(\left[0, T^{*}\right] ; \mathcal{C}^{1}[0,1]\right)^{2} \rightarrow \mathcal{C}^{0}\left(\left[0, T^{*}\right] ; \mathcal{C}^{1}[0,1]\right)^{2}$ defined and of class $\mathcal{C}^{1}$ on a neighborhood $\mathcal{O}$ of $(-1,1) \in \mathcal{C}^{0}\left(\left[0, T^{*}\right] ; \mathcal{C}^{1}[0,1]\right)^{2}$ satisfying

$$
\begin{gathered}
\theta \circ \Pi=0, \\
((\theta(\lambda)=0) \Rightarrow(\Pi(\lambda)=\lambda)) \forall \lambda \in \mathcal{O} .
\end{gathered}
$$

Let us point out that, at least for $\gamma$ small enough,

$$
\theta\left(-\sqrt{H^{\gamma}}, \sqrt{H^{\gamma}}\right)=0 .
$$

Indeed, for $|\gamma|$ small enough and for $\left(\lambda_{1}, \lambda_{2}\right)=\left(-\sqrt{H^{\gamma}}, \sqrt{H^{\gamma}}\right):=\lambda^{\gamma},(4.31)$ clearly holds and, as one easily checks,

$$
\begin{aligned}
& \tau_{1}^{\lambda^{\gamma}}=\tau_{3}^{\lambda^{\gamma}}=\tau_{0}+\frac{2}{\sqrt{1+(\gamma / 2)}+\sqrt{1-(\gamma / 2)}}, \\
& \tau_{2}^{\lambda^{\gamma}}=\tau_{4}^{\lambda^{\gamma}}=\tau_{0}+\frac{4}{\sqrt{1+(\gamma / 2)}+\sqrt{1-(\gamma / 2)}} .
\end{aligned}
$$

For $\nu>0$ and $\gamma \in(0,1 / 2]$, let $\mathcal{L}_{\nu, \gamma}$ be the set of $\lambda=\left(\lambda_{1}, \lambda_{2}\right) \in \mathcal{C}^{0}\left(\left[0, T^{*}\right] ; \mathcal{C}^{1}[0,1]\right)^{2}$ satisfying

$$
\left|\lambda_{1}+\sqrt{H^{\gamma}}\right|_{\mathcal{C}^{0}\left(\left[0, T^{*}\right] ; \mathcal{C}^{1}[0,1]\right)}+\left|\lambda_{2}-\sqrt{H^{\gamma}}\right|_{\mathcal{C}^{0}\left(\left[0, T^{*}\right] ; \mathcal{C}^{1}[0,1]\right)} \leqslant \nu
$$


Let $\mathcal{L}_{\nu, \gamma}^{0}$ be the set of $\lambda=\left(\lambda_{1}, \lambda_{2}\right) \in \mathcal{L}_{\nu, \gamma}$ such that $\theta(\lambda)=0$. By $(4.39,4.40)$ and $(4.41)$, one gets, since $\theta$ is of class $\mathcal{C}^{1}$, the existence of $\gamma_{6} \in(0,1)$ and of $C_{21}>0$ such that, for every $\gamma \in\left[-\gamma_{6}, \gamma_{6}\right]$ and for every $\nu \in\left[-\gamma_{6}, \gamma_{6}\right]$,

$$
\Pi\left(\mathcal{L}_{\nu, \gamma}\right) \subset \mathcal{L}_{C_{21} \nu, \gamma}^{0}
$$

For $\nu>0$ and $\gamma>0$ both small enough, let $\mathcal{H}_{\nu, \gamma}$ be the set of $(\lambda, \alpha, \beta, b, f)$ satisfying

$$
\begin{gathered}
\lambda=\left(\lambda_{1}, \lambda_{2}\right) \in \mathcal{L}_{\nu, \gamma}^{0} \\
\alpha=\left(\alpha^{1}, \alpha^{2}\right) \in \mathcal{C}^{0}([0,1])^{2}, \beta=\left(\beta^{1}, \beta^{2}\right) \in \mathcal{C}^{0}([0,1])^{2} \\
b=\left(b_{0}, b_{1}\right) \in \mathcal{C}^{0}\left(\left[0, T^{*}\right]\right)^{2}, f=\left(f_{1}, f_{2}\right) \in \mathcal{C}^{0}\left(\left[0, T^{*}\right] \times[0,1]\right)^{2} \\
\left(1+\frac{\gamma}{4}\right)\left(\alpha^{2}(0)-\alpha^{1}(0)\right)+b_{0}(0)=(1+\gamma)\left(\alpha^{2}(1)-\alpha^{1}(1)\right)+b_{1}(0) \\
\left(1+\frac{\gamma}{4}\right)\left(\beta^{2}(0)-\beta^{1}(0)\right)+b_{0}\left(T^{*}\right)=(1+\gamma)\left(\beta^{2}(1)-\beta^{1}(1)\right)+b_{1}\left(T^{*}\right)
\end{gathered}
$$

This set $\mathcal{H}_{\nu, \gamma}$ is equipped with the topology defined by the usual norm on $\mathcal{C}^{0}\left(\left[0, T^{*}\right] ; \mathcal{C}^{1}\left[0, T^{*}\right]\right)^{2} \times \mathcal{C}^{0}([0,1])^{4}$ $\times \mathcal{C}^{0}\left(\left[0, T^{*}\right]\right)^{2} \times \mathcal{C}^{0}\left(\left[0, T^{*}\right] \times[0,1]\right)^{2}$.

For every compact subset $K$ of $\mathbb{R}^{n}$, for every continuous maps $f: K \rightarrow \mathbb{R}, g=\left(g_{1}, \ldots, g_{m}\right): K \rightarrow \mathbb{R}^{m}$ and for every real number $\rho \geqslant 0$, let

$$
\begin{gathered}
\omega_{\rho}(f):=\operatorname{Max}\left\{\left|f(z)-f\left(z^{\prime}\right)\right| ; z \in K, z^{\prime} \in K,\left|z-z^{\prime}\right| \leqslant \rho\right\}, \\
\omega_{\rho}(g):=\sum_{i=1}^{m} \omega_{\rho}\left(g_{i}\right) .
\end{gathered}
$$

Let us assume that the following lemma, whose proof is postponed to Appendix A, holds.

Lemma 17. There exists $C_{22}>2$ and $\gamma_{7} \in\left(0, \gamma_{5}\right]$ such that, for every $\gamma \in\left(0, \gamma_{7}\right]$, there exists a continuous $\operatorname{map} \mathbf{W}_{\gamma}$

$$
\begin{aligned}
\mathcal{H}_{\gamma_{7} \gamma, \gamma} & \rightarrow \mathcal{C}^{0}\left(\left[0, T^{*}\right]\right) \\
(\lambda, \alpha, \beta, b, f) & \mapsto w=\mathbf{W}_{\gamma}(\lambda, \alpha, \beta, b, f),
\end{aligned}
$$

such that the three following properties hold.

(i) One has

$$
2 w(0)=\left(1+\frac{\gamma}{4}\right)\left(\alpha^{2}(0)-\alpha^{1}(0)\right)+b_{0}(0)=(1+\gamma)\left(\alpha^{2}(1)-\alpha^{1}(1)\right)+b_{1}(0)
$$

(ii) The unique solution $Z=\left(Z^{1}, Z^{2}\right) \in \mathcal{C}^{0}\left(\left[0, T^{*}\right] \times\left[0, T^{*}\right]\right)^{2}$ (which exists by the compatibility condition (4.50)) of

$$
\begin{gathered}
Z_{t}^{1}+\lambda_{1} Z_{x}^{1}=f_{1}, Z_{t}^{2}+\lambda_{2} Z_{x}^{2}=f_{2}, \\
\left(1+\frac{\gamma}{4}\right)\left(Z^{2}(t, 0)-Z^{1}(t, 0)\right)+b_{0}(t)=(1+\gamma)\left(Z^{2}(t, 1)-Z^{1}(t, 1)\right)+b_{1}(t)=2 w(t), \\
Z(0, \cdot)=\alpha,
\end{gathered}
$$


satisfies

$$
\begin{gathered}
Z\left(T^{*}, \cdot\right)=\beta, \\
\frac{1}{\gamma}\left|\int_{0}^{1}\left(Z^{1}+Z^{2}\right)(\cdot, x) \mathrm{d} x\right|_{0}+\left|Z^{1}\right|_{0}+\left|Z^{2}\right|_{0} \leqslant \frac{C_{22}}{\gamma}\left(|\alpha|_{0}+|\beta|_{0}+|b|_{0}+|f|_{0}\right), \\
\frac{1}{\gamma} \omega_{\rho}\left(\int_{0}^{1}\left(Z^{1}+Z^{2}\right)(\cdot, x) \mathrm{d} x\right)+\omega_{\rho}\left(Z^{1}, Z^{2}\right) \leqslant \frac{C_{22}}{\gamma}\left(\omega_{\rho}(\alpha, \beta, b, f)+\rho\left(|\alpha|_{0}+|\beta|_{0}+|b|_{0}+|f|_{0}\right)\right) \forall \rho \in[0,+\infty) .
\end{gathered}
$$

(iii) The map $\mathbf{W}_{\gamma}(\lambda, \cdot)$ is linear.

Let us recall that (see Rem. 12) the solutions of (4.51) have to be understood by using the corresponding system of integrals along the characteristics.

Lemma 17 is not sufficient to prove Proposition 5 by means of an iterative scheme: one has to deal with the fact that $\theta=0$ is required at each step and that one wants to steer $\delta$ from $\delta_{0}$ to $\delta_{1}$. We do it by considering special explicit $\left(Z^{1}, Z^{2}\right)$ which are "almost" (i.e. up to the order $\left.\gamma^{2}\right)$ solutions of

$$
\begin{gathered}
Z_{t}^{1}+\lambda^{1} Z_{x}=0, Z_{t}^{2}+\lambda^{2} Z_{x}=0 \\
\left(1+\frac{\gamma}{4}\right)\left(Z^{2}(t, 0)-Z^{1}(t, 0)\right)=(1+\gamma)\left(Z^{2}(t, 1)-Z^{1}(t, 1)\right) \\
Z^{1}(0, \cdot)=Z^{2}(0, \cdot)=Z^{1}\left(T^{*}, \cdot\right)=Z^{2}\left(T^{*}, \cdot\right)=0 .
\end{gathered}
$$

Let $g \in \mathcal{C}^{\infty}(\mathbb{R})$ be such that

$$
g=0 \in(-\infty, 1] \cup\left[T^{*}-1, \infty\right) .
$$

For $\gamma \in[-1,1]$, let $\zeta^{\gamma}=\left(\zeta^{\gamma, 1}, \zeta^{\gamma, 2}\right) \in \mathcal{C}^{\infty}([0, T *] \times[0,1])$ be defined by

$$
\begin{aligned}
\zeta^{\gamma, 1}(t, x) & :=\frac{2}{1+\frac{\gamma}{4}} g\left(t-1+x-\frac{\gamma}{4}\left(x-x^{2}\right)\right)-\frac{2}{1+\gamma} g\left(t+x-\frac{\gamma}{4}\left(x-x^{2}\right)\right), \\
\zeta^{\gamma, 2}(t, x) & :=\frac{2}{1+\frac{\gamma}{4}} g\left(t+1-x+\frac{\gamma}{4}\left(x-x^{2}\right)\right)-\frac{2}{1+\gamma} g\left(t-x+\frac{\gamma}{4}\left(x-x^{2}\right)\right) .
\end{aligned}
$$

Then, by (4.54),

$$
\zeta^{\gamma}(0, \cdot)=\zeta^{\gamma}\left(T^{*}, \cdot\right)=0
$$

Moreover,

$$
\begin{gathered}
\zeta_{t}^{\gamma, 1}-\frac{1}{1-\frac{\gamma}{4}(1-2 x)} \zeta_{x}^{\gamma, 1}=0, \\
\zeta_{t}^{\gamma, 2}+\frac{1}{1-\frac{\gamma}{4}(1-2 x)} \zeta_{x}^{\gamma, 2}=0, \\
\left(1+\frac{\gamma}{4}\right)\left(\zeta^{\gamma, 2}(t, 0)-\zeta^{\gamma, 1}(t, 0)\right)=(1+\gamma)\left(\zeta^{\gamma, 2}(t, 1)-\zeta^{\gamma, 1}(t, 1)\right)=2(g(t+1)-g(t-1)) .
\end{gathered}
$$

Note that there exists $C_{23}>0$, which does not depend on $g$ and $\gamma \in(-1,1)$, such that

$$
\left|\int_{0}^{1}\left(\zeta^{\gamma, 1}+\zeta^{\gamma, 2}\right)(\cdot, x) \mathrm{d} x\right|_{0} \leqslant C_{23} \gamma|g|_{\mathcal{C}^{1}\left(\left[1, T^{*}-1\right]\right)} .
$$

Straightforward estimates, together with (4.57) to (4.60), give the following lemma, whose proof is omitted: 
Lemma 18. There exists $\gamma_{8} \in(0,1]$ and $C_{24}>0$ such that, for every $\lambda \in \mathcal{L}_{\nu, \gamma}^{0}$, with $(\nu, \gamma) \in\left[0, \gamma_{8}\right]^{2}$ and for every $g \in \mathcal{C}^{\infty}(\mathbb{R})$ satisfying (4.54), the solution $Z=\left(Z^{1}, Z^{2}\right) \in C^{1}\left(\left[0, T^{*}\right] \times[0,1]\right)$ of

$$
\begin{gathered}
Z_{t}^{1}+\lambda_{1} Z_{x}^{1}=Z_{t}^{2}+\lambda_{2} Z_{x}^{2}=0, \\
\left(1+\frac{\gamma}{4}\right)\left(Z^{2}(t, 0)-Z^{1}(t, 0)\right)=(1+\gamma)\left(Z^{2}(t, 1)-Z^{1}(t, 1)\right)=2(g(t+1)-g(t-1)), \\
Z(0, \cdot)=0,
\end{gathered}
$$

satisfies

$$
\left|Z-\zeta^{\gamma}\right|_{1} \leqslant C_{24}\left(\nu+\gamma^{2}\right)|g|_{\mathcal{C}^{2}\left(\left[1, T^{*}-1\right]\right)} .
$$

In particular, with (4.57),

$$
\left|Z\left(T^{*}, \cdot\right)\right|_{1} \leqslant C_{24}\left(\nu+\gamma^{2}\right)|g|_{\mathcal{C}^{2}\left(\left[1, T^{*}-1\right]\right)}
$$

Note that, by (4.54),

$$
\begin{gathered}
\int_{0}^{T^{*}}(g(t+1)-g(t-1)) \mathrm{d} t=0 \\
\bar{\delta}:=\int_{0}^{T^{*}} \int_{0}^{t}\left(g\left(t_{1}+1\right)-g\left(t_{1}-1\right)\right) \mathrm{d} t_{1} \mathrm{~d} t=2 \int_{-\infty}^{+\infty} g(t) \mathrm{d} t .
\end{gathered}
$$

For $\gamma \in[-1,1]$, let us denote by $\mathbf{L}_{\gamma}$ the map

$$
\begin{aligned}
\mathcal{C}^{0}\left(\left[0, T^{*}\right] ; \mathcal{C}^{1}\left(\left[0, T^{*}\right]\right)\right)^{2} \times \mathcal{C}^{0}\left(\left[0, T^{*}\right]\right) & \rightarrow \mathcal{C}^{0}\left(\left[0, T^{*}\right] ; \mathcal{C}^{1}\left(\left[0, T^{*}\right]\right)\right)^{2} \\
\left(R^{1}, R^{2}, \sigma\right) & \mapsto\left(\lambda^{1}, \lambda^{2}\right)
\end{aligned}
$$

defined by

$$
\begin{aligned}
& \lambda_{1}:=-\frac{3 R^{1}}{4}+\frac{R^{2}}{4}-\sqrt{H^{\gamma}}-\sigma \\
& \lambda_{2}:=-\frac{R^{1}}{4}+\frac{3 R^{2}}{4}+\sqrt{H^{\gamma}}-\sigma .
\end{aligned}
$$

Let $\Theta: \mathbb{R} \times \mathcal{C}^{0}\left(\left[0, T^{*}\right] ; \mathcal{C}^{1}[0,1]\right)^{2} \times \mathcal{C}^{0}\left(\left[0, T^{*}\right]\right) \rightarrow \mathbb{R}^{2}$ be defined by

$$
\Theta\left(\gamma, R^{1}, R^{2}, \sigma\right):=\theta \circ \mathbf{L}_{\gamma}\left(R^{1}, R^{2}, \sigma\right) .
$$

The function $\Theta$ is of class $\mathcal{C}^{1}$ in a neighborhood of 0 in $\mathbb{R} \times \mathcal{C}^{0}\left(\left[0, T^{*}\right] ; \mathcal{C}^{1}([0,1])\right)^{2} \times \mathcal{C}^{0}\left(\left[0, T^{*}\right]\right)$. Let $R^{1 \sharp}$ and $R^{2 \sharp}$ be in $\mathcal{C}^{0}\left(\left[0, T^{*}\right] ; \mathcal{C}^{1}[0,1]\right)$. Let $\sigma^{\sharp}$ be in $\mathcal{C}^{0}\left(\left[0, T^{*}\right]\right)$. Then

$$
\Theta^{\prime}(0)\left(0, R^{1 \sharp}, R^{2 \sharp}, \sigma^{\sharp}\right)=\left(d_{1}, d_{2}\right),
$$

with

$$
\begin{gathered}
d_{1}=-\frac{1}{4} \int_{\frac{T^{*}}{2}-1}^{\frac{T^{*}}{2}}\left(\left(3 R^{1 \sharp}-R^{2 \sharp}\right)\left(t,-t+\frac{T^{*}}{2}\right)+\left(R^{1 \sharp}-3 R^{2 \sharp}\right)\left(t, t-\frac{T^{*}}{2}+1\right)+8 \sigma^{\sharp}(t)\right) \mathrm{d} t, \\
d_{2}=d_{1}-\frac{1}{4} \int_{\frac{T^{*}}{2}}^{\frac{T^{*}}{2}+1}\left(\left(3 R^{1 \sharp}-R^{2 \sharp}\right)\left(t,-t+\frac{T^{*}}{2}+1\right)+\left(R^{1 \sharp}-3 R^{2 \sharp}\right)\left(t, t-\frac{T^{*}}{2}\right)+8 \sigma^{\sharp}(t)\right) \mathrm{d} t .
\end{gathered}
$$


Let $g \in \mathcal{C}^{\infty}(\mathbb{R})$ and $\epsilon \in \mathbb{R}$. Consider $\zeta^{\gamma, \epsilon}$ as defined by (4.55) and (4.56) with $\epsilon g$ instead of $g$ in these equations. Thanks to Lemma 16, one can associate to $\zeta^{\gamma, \epsilon}$ a unique pair of continuous maps $\left(R^{\gamma, \epsilon}, \sigma^{\gamma, \epsilon}\right)$ with $R^{\gamma, \epsilon}=\left(R^{1, \gamma, \epsilon}, R^{2, \gamma, \epsilon}\right):\left[0, T^{*}\right] \times[0,1] \rightarrow \mathbb{R}^{2}, \sigma^{\gamma, \epsilon}:\left[0, T^{*}\right] \rightarrow \mathbb{R}$ such that

$$
\begin{gathered}
\left(\zeta^{\gamma, \epsilon}\right)^{\operatorname{tr}}=M_{3}\left(R_{x}^{\gamma, \epsilon}\right)^{\operatorname{tr}}-M_{4}\left(R^{\gamma, \epsilon}\right)^{\operatorname{tr}}, \\
R^{2, \gamma, \epsilon}(0)-R^{1, \gamma, \epsilon}(0)=R^{2, \gamma, \epsilon}(1)-R^{1, \gamma, \epsilon}(1)=2 \sigma^{\gamma, \epsilon}, \\
\int_{0}^{1} \frac{R^{1, \gamma, \epsilon}+R^{2, \gamma, \epsilon}}{2} \sqrt{H^{\gamma}}+\frac{\left(R^{1, \gamma, \epsilon}+R^{2, \gamma, \epsilon}\right)^{2}}{16} \mathrm{~d} x=0,
\end{gathered}
$$

at least if $\gamma \in\left[-\gamma_{5}, \gamma_{5}\right] \backslash\{0\}$ and $|\epsilon|$ are small enough. It turns out that, in this special case, such a $\left(R^{\gamma, \epsilon}, \sigma^{\gamma, \epsilon}\right)$ can be extended by continuity for $\gamma=0$ and $|\epsilon|$ small enough. Straightforward computations give the following lemma, whose proof is omitted:

Lemma 19. There exists $\epsilon_{g}>0$ such that there exists one and only one map of class $\mathcal{C}^{2}$

$$
\begin{aligned}
\left(-\epsilon_{g},-\epsilon_{g}\right)^{2} & \rightarrow \mathcal{C}^{2}\left(\left[0, T^{*}\right] \times[0,1]\right)^{2} \times \mathcal{C}^{2}\left(\left[0, T^{*}\right]\right) \\
(\gamma, \epsilon) & \mapsto\left(R^{\gamma, \epsilon}, \sigma^{\gamma, \epsilon}\right)
\end{aligned}
$$

such that, with $R^{\gamma, \epsilon}=\left(R^{1, \gamma, \epsilon}, R^{2, \gamma, \epsilon}\right)$, one has (4.69, 4.70) and (4.71). Moreover

$$
\begin{aligned}
R^{1, \sharp}(t, x) & :=\left(\frac{\partial R^{1,0, \epsilon}}{\partial \epsilon}\right)_{\epsilon=0}(t, x)=2 G(t-1+x)-2 G(t+x), \\
R^{2, \sharp}(t, x) & :=\left(\frac{\partial R^{2,0, \epsilon}}{\partial \epsilon}\right)_{\epsilon=0}(t, x)=2 G(t+1-x)-2 G(t-x), \\
\sigma^{\sharp}(t) & :=\left(\frac{\partial \sigma^{0, \epsilon}}{\partial \epsilon}\right)_{\epsilon=0}(t)=G(t+1)-G(t-1),
\end{aligned}
$$

with

$$
G(t):=\int_{-\infty}^{t} g\left(t_{1}\right) \mathrm{d} t_{1}
$$

From (4.67) and (4.68), one gets

$$
\begin{aligned}
d_{1}=3\left(G\left(\frac{T^{*}}{2}\right)-G\left(\frac{T^{*}}{2}-1\right)\right)+\frac{3}{2} \int_{\frac{T^{*}}{2}-2}^{\frac{T^{*}}{2}-1} G(t) \mathrm{d} t-\frac{3}{2} \int_{\frac{T^{*}}{2}}^{\frac{T^{*}}{2}+1} G(t) \mathrm{d} t, \\
d_{2}=-3\left(G\left(\frac{T^{*}}{2}+1\right)-2 G\left(\frac{T^{*}}{2}\right)+G\left(\frac{T^{*}}{2}-1\right)\right) \\
+\frac{3}{2} \int_{\frac{T^{*}}{2}-2}^{\frac{T^{*}}{2}-1} G(t) \mathrm{d} t-\frac{3}{2} \int_{\frac{T^{*}}{2}-1}^{\frac{T^{*}}{2}+1} G(t) \mathrm{d} t+\frac{3}{2} \int_{\frac{T^{*}}{2}+1}^{\frac{T^{*}}{2}+2} G(t) \mathrm{d} t .
\end{aligned}
$$


Let us now fix three functions $g^{0}, g^{1}, g^{2}$ in $\mathcal{C}^{\infty}(\mathbb{R})$ of support included in $\left[-1, T^{*}+1\right]$ such that, with $\bar{\delta}, d_{1}$ and $d_{2}$ defined by $(4.63,4.76)$ and $(4.77)$, one has

$$
\begin{aligned}
& \text { for } g=g^{0}, \quad\left(\bar{\delta}, d_{1}, d_{2}\right)=(1,0,0), \\
& \text { for } g=g^{1}, \quad\left(\bar{\delta}, d_{1}, d_{2}\right)=(0,1,0), \\
& \text { for } g=g^{2}, \quad\left(\bar{\delta}, d_{1}, d_{2}\right)=(0,0,1) .
\end{aligned}
$$

Let $w^{0}, w^{1}, w^{2}$ in $\mathcal{C}^{\infty}\left(\left[0, T^{*}\right]\right)$ be defined by

$$
\begin{aligned}
& w^{0}(t):=\left(g^{0}(t+1)-g^{0}(t-1)\right), \\
& w^{1}(t):=\left(g^{1}(t+1)-g^{1}(t-1)\right), \\
& w^{2}(t):=\left(g^{2}(t+1)-g^{2}(t-1)\right) .
\end{aligned}
$$

For $i \in\{0,1,2\}, \lambda \in \mathcal{L}_{\nu, \gamma}$ with $\nu>0$ and $\gamma$ both small enough, let us define $Z_{i, \lambda, \gamma}=\left(Z_{i, \lambda, \gamma}^{1}, Z_{i, \lambda, \gamma}^{2}\right)$ $\in C^{1}\left(\left[0, T^{*}\right] \times[0,1]\right)$ and $\beta_{i, \lambda, \gamma} \in C^{1}([0,1])^{2}$ by requiring

$$
\begin{gathered}
\left(Z_{i, \lambda, \gamma}^{1}\right)_{t}+\lambda_{1}\left(Z_{i, \lambda, \gamma}^{1}\right)_{x}=\left(Z_{i, \lambda, \gamma}^{2}\right)_{t}+\lambda_{2}\left(Z_{i, \lambda, \gamma}^{2}\right)_{x}=0 \\
\left(1+\frac{\gamma}{4}\right)\left(Z_{i, \lambda, \gamma}^{2}(t, 0)-Z_{i, \lambda, \gamma}^{1}(t, 0)\right)=(1+\gamma)\left(Z_{i, \lambda, \gamma}^{2}(t, 1)-Z_{i, \lambda, \gamma}^{1}(t, 1)\right)=2 w^{i}(t) \\
Z_{i, \lambda, \gamma}(0, \cdot)=0 \\
\beta_{i, \lambda, \gamma}=Z_{i, \lambda, \gamma}\left(T^{*}, \cdot\right) .
\end{gathered}
$$

For $Y_{0}=\left(H_{0}, v_{0}, s_{0}, D_{0}\right) \in \mathcal{Y}$ and $\gamma \in(-1,1)$, let us define $\left(R_{0 \gamma}^{1}, R_{0 \gamma}^{2}\right) \in \mathcal{C}^{1}([0,1])^{2}, \sigma_{0} \in \mathbb{R}, \delta_{0} \in \mathbb{R}$ and $\alpha_{\gamma}=\left(\alpha_{\gamma}^{1}, \alpha_{\gamma}^{2}\right) \in \mathcal{C}^{0}([0,1])^{2}$ by (see $(4.1,4.2,4.5)$ and $\left.(4.14)\right)$

$$
\begin{gathered}
\delta_{0}:=D_{0}, \\
\sigma_{0}:=s_{0}, \\
R_{0 \gamma}^{1}:=-v_{0}+2 \sqrt{H_{0}}-2 \sqrt{H^{\gamma}}-\sigma_{0} \\
R_{0 \gamma}^{2}:=v_{0}+2 \sqrt{H_{0}}-2 \sqrt{H^{\gamma}}+\sigma_{0} \\
\left(\begin{array}{c}
\alpha_{\gamma}^{1} \\
\alpha_{\gamma}^{2}
\end{array}\right):=\left(\begin{array}{cc}
1-\frac{5 \gamma}{4} x & 0 \\
0 & -1+\frac{5 \gamma}{4} x
\end{array}\right)\left(\begin{array}{l}
R_{0 \gamma x}^{1} \\
R_{0 \gamma x}^{2}
\end{array}\right)-\gamma\left(\begin{array}{c}
\frac{5}{8}-\frac{1}{8} \\
\frac{1}{8}-\frac{5}{8}
\end{array}\right)\left(\begin{array}{l}
R_{0 \gamma}^{1} \\
R_{0 \gamma}^{2}
\end{array}\right) .
\end{gathered}
$$

Let us point out that, by $(1.8-1.10,4.19,4.20)$ and (4.84) to $(4.88)$, one has

$$
\begin{aligned}
&\left(1+\frac{\gamma}{4}\right)\left(\alpha_{\gamma}^{2}(0)-\alpha_{\gamma}^{1}(0)\right)+B_{0}\left(\gamma, R_{0 \gamma}(0), R_{0 \gamma x}(0)\right)=(1+\gamma)\left(\alpha_{\gamma}^{2}(1)-\alpha_{\gamma}^{1}(1)\right) \\
&+B_{1}\left(\gamma, R_{0 \gamma}(1), R_{0 \gamma x}(1)\right), \\
& R_{0 \gamma}^{2}(0)-R_{0 \gamma}^{1}(0)=R_{0 \gamma}^{2}(1)-R_{0 \gamma}^{1}(1)=2 \sigma_{0} \\
& \int_{0}^{1}\left(R_{0 \gamma}^{1}+R_{0 \gamma}^{2}\right) \sqrt{H^{\gamma}}+\frac{\left(R_{0 \gamma}^{1}+R_{0 \gamma}^{2}\right)^{2}}{8} \mathrm{~d} x=0 .
\end{aligned}
$$


Similarly, for $Y_{1}=\left(H_{1}, v_{1}, s_{1}, D_{1}\right) \in \mathcal{Y}$, we define $\left(R_{1 \gamma}^{1}, R_{1 \gamma}^{2}\right) \in \mathcal{C}^{1}([0,1])^{2}, \sigma_{1 \gamma} \in \mathbb{R}, \delta_{1 \gamma} \in \mathbb{R}$ and $\beta_{\gamma}=\left(\beta_{\gamma}^{1}, \beta_{\gamma}^{2}\right) \in$ $\mathcal{C}^{0}([0,1])^{2}$ by (see $(4.1,4.2,4.5)$ and $\left.(4.14)\right)$

$$
\begin{gathered}
\delta_{1 \gamma}:=D_{1}-\frac{\gamma T^{* 2}}{2}, \\
\sigma_{1 \gamma}:=s_{1}-\gamma T^{*}, \\
R_{1 \gamma}^{1}:=-v_{1}+2 \sqrt{H_{1}}-2 \sqrt{H^{\gamma}}-\sigma_{1 \gamma}, \\
R_{1 \gamma}^{2}:=v_{1}+2 \sqrt{H_{1}}-2 \sqrt{H^{\gamma}}+\sigma_{1 \gamma}, \\
\left(\begin{array}{c}
\beta_{\gamma}^{1} \\
\beta_{\gamma}^{2}
\end{array}\right):=\left(\begin{array}{cc}
1-\frac{5 \gamma}{4} x & 0 \\
0 & -1+\frac{5 \gamma}{4} x
\end{array}\right)\left(\begin{array}{l}
R_{1 \gamma x}^{1} \\
R_{1 \gamma x}^{2}
\end{array}\right)-\gamma\left(\begin{array}{l}
\frac{5}{8}-\frac{1}{8} \\
\frac{1}{8}-\frac{5}{8}
\end{array}\right)\left(\begin{array}{l}
R_{1 \gamma}^{1} \\
R_{1 \gamma}^{2}
\end{array}\right) .
\end{gathered}
$$

Again, by $(1.8-1.10,4.19,4.20)$ and $(4.92)$ to $(4.96)$, one has

$$
\begin{aligned}
\left(1+\frac{\gamma}{4}\right)\left(\beta_{\gamma}^{2}(0)\right. & \left.-\beta_{\gamma}^{1}(0)\right)+B_{0}\left(\gamma, R_{1 \gamma}(0), R_{1 \gamma x}(0)\right)=(1+\gamma)\left(\beta_{\gamma}^{2}(1)-\beta_{\gamma}^{1}(1)\right) \\
& +B_{1}\left(\gamma, R_{1 \gamma}(1), R_{1 \gamma x}(1)\right), \\
R_{1 \gamma}^{2}(0) & -R_{1 \gamma}^{1}(0)=R_{1 \gamma}^{2}(1)-R_{1 \gamma}^{1}(1)=2 \sigma_{1 \gamma}, \\
\int_{0}^{1}\left(R_{1 \gamma}^{1}+R_{1 \gamma}^{2}\right) \sqrt{H^{\gamma}} & +\frac{\left(R_{1 \gamma}^{1}+R_{1 \gamma}^{2}\right)^{2}}{8} \mathrm{~d} x=0 .
\end{aligned}
$$

We let

$$
\chi_{\gamma}:=\delta_{1 \gamma}-\delta_{0}-\sigma_{0} T^{*}
$$

For $\nu \in\left(0, \gamma_{5}\right]$ and $\gamma \in\left(0, \gamma_{5}\right]$, let $\mathcal{E}_{\nu, \gamma}\left(Y_{0}, Y_{1}\right)$ be the set of $\left(Z^{1}, Z^{2}\right)$ in $\mathcal{C}^{0}\left(\left[0, T^{*}\right] \times[0,1]\right)^{2}$ satisfying

$$
\begin{gathered}
\frac{1}{\gamma}\left|\int_{0}^{1}\left(Z^{1}+Z^{2}\right)(\cdot, x) \mathrm{d} x\right|_{0}+\left|Z^{1}\right|_{0}+\left|Z^{2}\right|_{0}+|\delta|_{0} \leqslant \nu, \\
Z(0, \cdot)=\alpha_{\gamma}, Z\left(T^{*}, \cdot\right)=\beta_{\gamma} .
\end{gathered}
$$

For $\nu>0$, let

$$
\mathcal{B}_{\nu}:=\left\{q=\left(q^{0}, q^{1}, q^{2}\right) \in \mathbb{R}^{3} ;\left|q^{0}\right|+\left|q^{1}\right|+\left|q^{2}\right| \leqslant \nu\right\} .
$$

Let us define a map

$$
\begin{gathered}
\mathbf{F}: \mathcal{E}_{\nu, \gamma}\left(Y_{0}, Y_{1}\right) \times \mathcal{B}_{\nu} \rightarrow \mathcal{C}^{0}\left(\left[0, T^{*}\right] \times[0,1]\right)^{2} \times \mathbb{R}^{3} \\
(Z, q)=\left(Z^{1}, Z^{2}, q^{0}, q^{1}, q^{2}\right) \mapsto(\bar{Z}, \bar{q})=\left(\bar{Z}^{1}, \bar{Z}^{2}, \bar{q}^{0}, \bar{q}^{1}, \bar{q}^{2}\right),
\end{gathered}
$$

by the following five-steps procedure: 
Step 1. One first define $R=\left(R^{1}, R^{2}\right) \in \mathcal{C}^{0}\left(\left[0, T^{*}\right] ; \mathcal{C}^{1}([0,1])\right)^{2}, \sigma \in \mathcal{C}^{0}\left(\left[0, T^{*}\right]\right), f=\left(f^{1}, f^{2}\right) \in \mathcal{C}^{0}\left(\left[0, T^{*}\right]\right.$ $\times[0,1])^{2}, b=\left(b_{0}, b_{1}\right) \in \mathcal{C}^{0}\left(\left[0, T^{*}\right]\right)^{2}$ by

$$
\begin{aligned}
(R, \sigma) & :=\left(\mathbf{R}^{\gamma}\left(Z^{1}, Z^{2}\right), \mathbf{S}^{\gamma}\left(Z^{1}, Z^{2}\right)\right), \\
b_{0} & :=B_{0}\left(\gamma, R(\cdot, 0), R_{x}(\cdot, 0)\right), \\
b_{1} & :=B_{1}\left(\gamma, R(\cdot, 0), R_{x}(\cdot, 0)\right), \\
& f_{1}:=F_{1}\left(x, \gamma, \sigma, R, R_{x}\right), \\
& f_{2}:=F_{2}\left(x, \gamma, \sigma, R, R_{x}\right) .
\end{aligned}
$$

Step 2. We define $\lambda=\left(\lambda_{1}, \lambda_{2}\right) \in \mathcal{C}^{0}\left(\left[0, T^{*}\right] ; \mathcal{C}^{1}([0,1])\right)^{2}$ by

$$
\lambda:=\Pi\left(\mathbf{L}_{\gamma}(R, \sigma)\right) .
$$

Step 3. With these $\lambda, b_{0}, b_{1}, f_{1}, f_{2}$, we define $w \in \mathcal{C}^{0}\left(\left[0, T^{*}\right]\right)$ by

$$
w:=\mathbf{W}_{\gamma}\left(\lambda, \alpha_{\gamma}, \beta_{\gamma}-q^{0} \beta_{0, \lambda, \gamma}-q^{1} \beta_{1, \lambda, \gamma}-q^{2} \beta_{2, \lambda, \gamma}, b, f\right)+q^{0} w^{0}+q^{1} w^{1}+q^{2} w^{2} .
$$

Step 4. Then $\bar{Z}=\left(\bar{Z}^{1}, \bar{Z}^{2}\right)$ is the unique continuous solution of

$$
\begin{gathered}
\bar{Z}_{t}^{1}+\lambda_{1} \bar{Z}_{x}^{1}=f_{1}, \bar{Z}_{t}^{2}+\lambda_{2} \bar{Z}_{x}^{2}=f_{2}, \\
\left(1+\frac{\gamma}{4}\right)\left(\bar{Z}^{2}(t, 0)-\bar{Z}^{1}(t, 0)\right)+b_{0}(t)=(1+\gamma)\left(\bar{Z}^{2}(t, 1)-\bar{Z}^{1}(t, 1)\right)+b_{1}(t)=2 w(t), \\
Z(0, \cdot)=\alpha_{\gamma},
\end{gathered}
$$

Step 5. Finally, $\left(\bar{q}^{0}, \bar{q}^{1}, \bar{q}^{2}\right)$ is defined by

$$
\begin{gathered}
\bar{q}^{0}:=\chi_{\gamma}+q^{0}-\int_{0}^{T^{*}} \int_{0}^{t_{1}} w\left(t_{1}\right) \mathrm{d} t_{1} \mathrm{~d} t, \\
\left(\bar{q}^{1}, \bar{q}^{2}\right):=\left(q^{1}, q^{2}\right)-\Theta\left(\gamma, \mathbf{R}^{\gamma}(\bar{Z}), \mathbf{S}^{\gamma}(\bar{Z})\right) .
\end{gathered}
$$

From Lemma 16 and straightforward estimates, one gets the following lemma, whose proof is omitted:

Lemma 20. There exists $C_{25}>0$ such that, for every $\gamma \in\left[-\gamma_{5}, \gamma_{5}\right] \backslash\{0\}$, for every $Z=\left(Z^{1}, Z^{2}\right) \in \mathcal{C}^{0}([0,1])^{2}$ with

$$
\frac{1}{\gamma}\left|\int_{0}^{1} Z^{1}+Z^{2} \mathrm{~d} x\right|+|Z|_{0} \leqslant \gamma_{5}
$$

one has

$$
\omega_{\rho}\left(\mathbf{R}^{\gamma}, \mathbf{S}^{\gamma}\right) \leqslant C_{25}\left(\frac{1}{\gamma} \omega_{\rho}\left(\int_{0}^{1}\left(Z^{1}+Z^{2}\right)(\cdot, x) \mathrm{d} x\right)+\omega_{\rho}(Z)+\rho|Z|_{0}\right)
$$

From $(4.21)$ to $(4.27,4.61,4.78)$ to $(4.80,4.89,4.97)$, Lemma 16 to Lemma 20 and straightforward estimates, one gets the following lemma, whose proof is omitted: 
Lemma 21. There exists $\gamma_{9} \in(0,1]$, and $C_{26}>0$ such that, for every $\nu \in\left(0, \gamma_{9}\right]$, for every $\gamma \in(0, \nu]$ and for every $\left(Y_{0}, Y_{1}\right) \in \mathcal{Y}$ with $\left|Y_{0}-Y^{\gamma}(0)\right|+\left|Y_{1}-Y^{\gamma}\left(T^{*}\right)\right| \leqslant \nu^{2} \gamma^{2}$,

$$
\mathcal{E}_{\nu \gamma, \gamma}\left(Y_{0}, Y_{1}\right) \text { is not empty, }
$$

$\mathbf{F}$ is well-defined and continuous on $\mathcal{E}_{\nu \gamma, \gamma}\left(Y_{0}, Y_{1}\right) \times \mathcal{B}_{\nu^{3 / 2} \gamma}$,

$$
\mathbf{F}\left(\mathcal{E}_{\nu \gamma, \gamma}\left(Y_{0}, Y_{1}\right) \times \mathcal{B}_{\nu^{3 / 2} \gamma}\right) \subset \mathcal{E}_{C_{26} \nu^{3 / 2} \gamma, \gamma} \times \mathcal{B}_{C_{26} \nu^{2} \gamma}
$$

and, for every $(Z, q) \in \mathcal{E}_{\nu \gamma, \gamma}\left(Y_{0}, Y_{1}\right) \times \mathcal{B}_{\nu \gamma}$ and for every $\rho \in[0,+\infty)$,

$$
\begin{aligned}
\frac{1}{\gamma} \omega_{\rho}\left(\int_{0}^{1}\left(\bar{Z}^{1}+\bar{Z}^{2}\right)(\cdot, x) \mathrm{d} x\right)+\omega_{\rho}\left(\bar{Z}^{1}, \bar{Z}^{2}\right) \\
\leqslant C_{26}\left(\nu\left(\frac{1}{\gamma} \omega_{\rho}\left(\int_{0}^{1}\left(Z^{1}+Z^{2}\right)(\cdot, x) \mathrm{d} x\right)+\omega_{\rho}\left(Z^{1}, Z^{2}\right)+\rho\right)+\frac{1}{\gamma} \omega_{\rho}\left(\alpha_{\gamma}, \beta_{\gamma}\right)\right)
\end{aligned}
$$

where

$$
(\bar{Z}, \bar{q})=\mathbf{F}(Z, q)
$$

Let

$$
\gamma_{10} \in\left(0, \operatorname{Min}\left(\gamma_{9}, \frac{1}{2 C_{26}}, \frac{1}{C_{26}^{2}}\right)\right] .
$$

Let $\gamma \in\left(0, \gamma_{10}\right]$, and $\left(Y_{0}, Y_{1}\right) \in \mathcal{Y}$ with $\left|Y_{0}-Y^{\gamma}(0)\right|+\left|Y_{1}-Y^{\gamma}\left(T^{*}\right)\right| \leqslant \gamma_{10}^{2} \gamma^{2}$. We claim that $\mathbf{F}$ has a fixed point in $\mathcal{E}_{\gamma_{10}, \gamma} \times \mathcal{B}_{\gamma_{10}^{3 / 2} \gamma}$. Let $\Omega \in \mathcal{C}^{0}([0,1] ;[0,+\infty))$ be such that

$$
\begin{gathered}
\Omega(0)=0, \\
\Omega(\rho) \geqslant \frac{2 C_{26}}{\gamma} \omega_{\rho}\left(\alpha_{\gamma}, \beta_{\gamma}\right)+\rho \forall \rho \in[0,+\infty)
\end{gathered}
$$

and such that the set $\mathcal{K}$ of $\left(Z^{1}, Z^{2}, q\right) \in \mathcal{E}_{\gamma_{10}, \gamma} \times \mathcal{B}_{\gamma_{10}^{3 / 2} \gamma}$ satisfying

$$
\frac{1}{\gamma} \omega_{\rho}\left(\int_{0}^{1}\left(Z^{1}+Z^{2}\right)(\cdot, x) \mathrm{d} x\right)+\omega_{\rho}\left(Z^{1}, Z^{2}\right) \leqslant \Omega(\rho) \forall \rho \in[0,+\infty)
$$

is not empty. Then, $\mathcal{K}$ is a nonempty convex compact subset of $\mathcal{C}^{0}\left(\left[0, T^{*}\right] \times[0,1]\right)^{2} \times \mathbb{R}^{3}$, and, by $(4.115-4.117)$ and (4.119),

$$
\mathbf{F}(\mathcal{K}) \subset \mathcal{K}
$$

Hence, by the Leray-Schauder fixed point theorem, $\mathbf{F}$ has a fixed point in $\mathcal{K}$.

Let us now check that the existence of a fixed point to $\mathbf{F}$ in $\mathcal{E}_{\gamma_{10} \gamma, \gamma} \times \mathcal{B}_{\gamma_{10}^{3 / 2} \gamma}$ implies Proposition 5. Let $\left(Z^{1}, Z^{2}, q\right)$ be a fixed point of $\mathbf{F}$ in $\mathcal{E}_{\gamma_{10} \gamma, \gamma} \times \mathcal{B}_{\gamma_{10}^{3 / 2}}$. Let $R=\left(R^{1}, R^{2}\right) \in \mathcal{C}^{0}\left(\left[0, T^{*}\right] ; \mathcal{C}^{1}[0,1]\right)^{2}$ and $\sigma \in \mathcal{C}^{0}\left(\left[0, T^{*}\right]\right)$ be defined by

$$
(R, \sigma):=\left(\mathbf{R}^{\gamma}\left(Z^{1}, Z^{2}\right), \mathbf{S}^{\gamma}\left(Z^{1}, Z^{2}\right)\right) .
$$

Using the fact that one has $\bar{q}=q$ in (4.112), one gets that

$$
\Theta(\gamma, R, \sigma)=0 .
$$


In particular $\lambda$ defined by (4.106) satisfies

$$
\lambda=\mathbf{L}_{\gamma}(R, \sigma)
$$

Let $w$ be defined by (4.107). Note that, by (4.111) with $\bar{q}^{0}=q^{0}$, one has

$$
\chi_{\lambda, \gamma}=\int_{0}^{T^{*}} \int_{0}^{t_{1}} w\left(t_{1}\right) \mathrm{d} t_{1} \mathrm{~d} t
$$

Moreover (4.107), together with (4.108) to (4.110) (with $\bar{Z}=Z$ ), implies that

$$
Z(0, \cdot)=\alpha_{\gamma}, Z\left(T^{*}, \cdot\right)=\beta_{\gamma}
$$

Let $\phi=\left(\phi^{1}, \phi^{2}\right) \in \mathcal{C}^{0}\left(\left[0, T^{*}\right] \times[0,1]\right)^{2}$ be defined by

$$
\phi^{\operatorname{tr}}(t, x):=R^{\operatorname{tr}}(t, x)-R^{\operatorname{tr}}(0, x)+\int_{0}^{t}\left(\Lambda R_{x}^{\operatorname{tr}}-M_{1} R^{\operatorname{tr}}-\sigma M_{2}\right)\left(t_{1}, x\right) \mathrm{d} t_{1} .
$$

Proposition 5 is proved if one checks that

$$
\begin{gathered}
\phi^{1}=\phi^{2}=0, \\
\dot{\sigma}=w .
\end{gathered}
$$

(Indeed the estimate (2.15), with $C_{1}$ large enough, follows from Lem. 16 if $\gamma_{10}>0$ is small enough.) From (4.11, $4.12,4.14-4.17)$ and $(4.125)$, one gets

$$
\left(\begin{array}{cc}
1-\frac{5 \gamma}{4} x & 0 \\
0 & -1+\frac{5 \gamma}{4} x
\end{array}\right)\left(\begin{array}{l}
\phi_{x}^{1} \\
\phi_{x}^{2}
\end{array}\right)-\gamma\left(\begin{array}{cc}
\frac{5}{8} & -\frac{1}{8} \\
\frac{1}{8} & -\frac{5}{8}
\end{array}\right)\left(\begin{array}{l}
\phi^{1} \\
\phi^{2}
\end{array}\right)=0
$$

By (4.120), one has (4.7). From $(4.3,4.4,4.7,4.11,4.12,4.14,4.15,4.18-4.20)$ and (4.125), one gets

$$
\phi^{2}(t, 0)-\phi^{1}(t, 0)=\phi^{2}(t, 1)-\phi^{1}(t, 1)=2\left(\sigma(t)-\sigma_{0}-\int_{0}^{t} w\left(t_{1}\right) \mathrm{d} t_{1}\right) .
$$

Define (see (4.1) and (4.2))

$$
\begin{gathered}
v:=\frac{R^{2}-R^{1}}{2}-\sigma \\
H:=\frac{\left(R^{1}+R^{2}\right)^{2}}{16}+\sqrt{H^{\gamma}} \frac{R^{1}+R^{2}}{2}+H^{\gamma} .
\end{gathered}
$$

Using $(4.3,4.4,4.11,4.12,4.125,4.130)$ and $(4.131)$, one gets

$$
\begin{aligned}
H(t, \cdot)-H(0, \cdot) & +\int_{0}^{t}(H v)_{x} \mathrm{~d} t_{1}=\left(\frac{\sqrt{H^{\gamma}}}{2}+\frac{R^{1}+R^{2}}{8}-\frac{\phi^{1}+\phi^{2}}{16}\right)\left(\phi^{1}+\phi^{2}\right) \\
& +\int_{0}^{t}\left(\lambda_{1} R_{1 x}+\lambda_{2} R_{2 x}-\frac{\gamma}{\sqrt{H^{\gamma}}} R^{2}+\frac{\gamma}{\sqrt{H^{\gamma}}} R^{1}+\frac{2 \gamma \sigma}{\sqrt{H^{\gamma}}}\right) \frac{\phi^{1}+\phi^{2}}{8} \mathrm{~d} t_{1} .
\end{aligned}
$$


By (4.120) and (4.131),

$$
\int_{0}^{1}(H(t, x)-H(0, x)) \mathrm{d} x=0
$$

By (4.7) and (4.130),

$$
v(\cdot, 0)=v(\cdot, 1)=0 .
$$

Using (4.132-4.134) and an integration by parts, one gets

$$
\int_{0}^{1}\left(\left(\phi^{1}+\phi^{2}\right) M\right)(x, t) \mathrm{d} x=\int_{0}^{t} \int_{0}^{1}\left(\left(\phi^{1}+\phi^{2}\right) N\right)\left(x, t_{1}\right) \mathrm{d} x \mathrm{~d} t_{1}
$$

with

$$
\begin{gathered}
M:=\sqrt{H^{\gamma}}+\frac{R^{1}+R^{2}}{4}-\frac{\phi^{1}+\phi^{2}}{8} \\
N:=-\frac{1}{4}\left(\lambda_{1} R_{1 x}+\lambda_{2} R_{2 x}\right)+\frac{2 \gamma}{\sqrt{H^{\gamma}}} R^{2}-\frac{2 \gamma}{\sqrt{H^{\gamma}}} R^{1}-\frac{4 \gamma \sigma}{\sqrt{H^{\gamma}}} .
\end{gathered}
$$

Straightforward estimates give the following lemma:

Lemma 22. There exists $\gamma_{11}>0$ such that, for every $\gamma \in\left(0, \gamma_{11}\right]$, there exists $C_{\gamma}>0$ such that for every $k \in \mathcal{C}^{0}([0,1])$ satisfying $|k-1|_{0} \leqslant \gamma_{11}$ and for every $\left(\psi^{1}, \psi^{2}\right) \in \mathcal{C}^{1}([0,1])^{2}$ satisfying

$$
\begin{gathered}
\left(\begin{array}{cc}
1-\frac{5 \gamma}{4} x & 0 \\
0 & -1+\frac{5 \gamma}{4} x
\end{array}\right)\left(\begin{array}{l}
\psi_{x}^{1} \\
\psi_{x}^{2}
\end{array}\right)-\gamma\left(\begin{array}{c}
\frac{5}{8}-\frac{1}{8} \\
\frac{1}{8}-\frac{5}{8}
\end{array}\right)\left(\begin{array}{l}
\psi^{1} \\
\psi^{2}
\end{array}\right)=0 \\
\psi^{2}(1)-\psi^{1}(1)=\psi^{2}(0)-\psi^{1}(0)
\end{gathered}
$$

one has

$$
\left|\psi^{1}\right|_{0}+\left|\psi^{2}\right|_{0} \leqslant C_{\gamma}\left|\int_{0}^{1}\left(\psi^{1}+\psi^{2}\right) k\right|
$$

Let $e \in \mathcal{C}^{0}\left(\left[0, T^{*}\right]\right)$ be defined by

Note that (4.125)

$$
e(t):=\left|\phi^{1}(t, \cdot)\right|_{0}+\left|\phi^{2}(t, \cdot)\right|_{0}
$$

$$
e(0)=0
$$

Let us apply (see also (4.128)) Lemma 22 with

$$
\begin{gathered}
\psi^{1}:=\phi^{1}(t, \cdot), \psi^{2}:=\phi^{2}(t, \cdot), \\
k:=M(t, \cdot) .
\end{gathered}
$$

One gets the existence of $C_{\gamma}^{\prime}>0$ such that at least for $\gamma_{10}$ small enough,

$$
e(t) \leqslant C_{\gamma}^{\prime} \int_{0}^{t} e\left(t_{1}\right) \mathrm{d} t_{1}
$$

From $(4.138,4.139)$ and Gronwall's lemma, one gets $e=0$, which, with (4.129), gives (4.126) and (4.127). 
Remark 23. It is in fact reasonable to conjecture that, at least for large enough $\mathrm{n}, \mathbf{F}^{n}$ is a contracting map for the $\mathcal{C}^{0}\left(\left[0, T^{*}\right] ; W^{-1, \infty}(0,1)\right) \times \mathbb{R}^{3}$-norm. Indeed such a phenomenon appears in the proof of the existence of the solution to the Cauchy problem when there is no control; see for example [12] (pp. 54-55) or [19] (pp. 71-72). If this is the case, $\mathbf{F}$ has a unique fixed point, which can be obtained as the limit of the iterative scheme $\left(Z_{n+1}, q_{n+1}\right)=\mathbf{F}^{n}\left(Z_{n}, q_{n}\right)$. Compare also, for the control of the Euler incompressible inviscid fluids [3], where the Leray-Schauder fixed point theorem was also used, with [11] where a contraction argument was used.

\section{Appendix A. Proof of Lemma 17}

For $\gamma \in(0,1 / 2]$, let $\mathcal{A}_{\gamma}^{-}$be the set of $\alpha=\left(\alpha^{1}, \alpha^{2}\right) \in \mathcal{C}^{0}([0,1])^{2}$ satisfying

$$
\alpha^{2}(0)=0,-\left(1+\frac{\gamma}{4}\right) \alpha^{1}(0)=(1+\gamma)\left(\alpha^{2}(1)-\left(\alpha^{1}(1)\right)\right.
$$

The set $\mathcal{A}_{\gamma}^{-}$is a closed linear subset of $\mathcal{C}^{0}([0,1])^{2}$.

Let us first prove the following lemma:

Lemma 24. There exists $\gamma_{12} \in(0,1 / 2]$ and $C_{27}$ such that, for every $\gamma \in\left(0, \gamma_{12}\right]$, there exists a continuous $\operatorname{map} \mathbf{W}_{\gamma}^{0}$

$$
\begin{gathered}
\mathcal{L}_{\gamma_{12 \gamma}, \gamma}^{0} \times \mathcal{A}_{\gamma}^{-} \rightarrow C^{0}\left(\left[\tau_{0}, T^{*}\right]\right) \\
(\lambda, \alpha)=\left(\left(\lambda_{1}, \lambda_{2}\right),\left(\alpha^{1}, \alpha^{2}\right)\right) \mapsto w=\mathbf{W}_{\gamma}^{0}(\lambda, \alpha),
\end{gathered}
$$

such that the following three properties hold.

(i) One has

$$
2 w\left(\tau_{0}\right)=-\left(1+\frac{\gamma}{4}\right) \alpha^{1}(0)
$$

and the solution $z=\left(z^{1}, z^{2}\right) \in \mathcal{C}\left(\left[\tau_{0}, T^{*}\right] \times[0,1]\right)^{2}$ (which exists by the compatibility condition (A.1)) of

$$
\begin{gathered}
z_{t}^{1}+\lambda_{1} z_{x}^{1}=z_{t}^{2}+\lambda_{2} z_{x}^{2}=0 \\
\left(1+\frac{\gamma}{4}\right)\left(z^{2}(t, 0)-z^{1}(t, 0)\right)=(1+\gamma)\left(z^{2}(t, 1)-z^{1}(t, 1)\right)=2 w(t), \\
z^{1}\left(\tau_{0}, \cdot\right)=\alpha^{1}, z^{2}\left(\tau_{0}, \cdot\right)=\alpha^{2}
\end{gathered}
$$

satisfies

$$
\begin{gathered}
z^{1}\left(T^{*}, \cdot\right)=0, z^{2}\left(T^{*}, \cdot\right)=0 . \\
\left|\int_{0}^{1}\left(z^{1}+z^{2}\right)(\cdot, x) \mathrm{d} x\right|_{0} \leqslant C_{27}|\alpha|_{0}, \\
\omega_{\rho}\left(\int_{0}^{1}\left(Z^{1}+Z^{2}\right)(\cdot, x) \mathrm{d} x\right) \leqslant C_{27} \rho|\alpha|_{0} \forall \rho \in[0,+\infty) .
\end{gathered}
$$

(ii) The following inequalities hold for every $\alpha=\left(\alpha^{1}, \alpha^{2}\right) \in \mathcal{A}_{\gamma}^{-}$

$$
\begin{gathered}
|w|_{0} \leqslant \frac{C_{27}}{\gamma}|\alpha|_{0}, \\
\omega_{\rho}(w) \leqslant \frac{C_{27}}{\gamma}\left(\omega_{\rho}(\alpha)+|\alpha|_{0} \rho\right) \forall \rho \in[0,+\infty) .
\end{gathered}
$$


(iii) The $\operatorname{map} \mathbf{W}_{\gamma}^{0}(\lambda, \cdot)$ is linear.

Let $\tau^{\prime} \in\left(0, \operatorname{Min}\left(1 / 2,\left(T^{*} / 2\right)-1\right)\right)$. Let $\mathcal{A}^{+}$be the set of $\alpha=\left(\alpha^{1}, \alpha^{2}\right) \in \mathcal{C}^{0}([0,1])^{2}$ satisfying

$$
\alpha^{2}(0)-\alpha^{1}(0)=\alpha^{1}(0)-\alpha^{1}(1)=0 .
$$

Let $\kappa \in \mathcal{C}^{1}(\mathbb{R})$ be such that

$$
\kappa=1 \text { on }(-\infty, 0], \kappa=0 \text { on }\left[\tau^{\prime},+\infty\right) .
$$

For $\nu>0$ and $\gamma \in(0,1 / 2]$ and $\lambda=\left(\lambda_{1}, \lambda_{2}\right) \in \mathcal{L}_{\nu, \gamma}^{0}$, let $\tau_{4}^{\lambda}$ be defined by $(4.35,4.36)$ and let

$$
\begin{aligned}
\mathbf{P}_{\lambda, \gamma}^{+}: \mathcal{A}_{\gamma}^{-} & \rightarrow \mathcal{A}^{+} \\
\left(\alpha_{-}^{1}, \alpha_{-}^{2}\right) & \mapsto\left(\alpha_{+}^{1}, \alpha_{+}^{2}\right),
\end{aligned}
$$

and

$$
\begin{aligned}
\mathbf{W}_{\lambda, \gamma}^{+}: \mathcal{A}_{\gamma}^{-} & \rightarrow \mathcal{C}^{0}\left(\left[\tau_{0}, \tau_{4}^{\lambda}+\tau^{\prime}\right]\right) \\
\left(\alpha_{-}^{1}, \alpha_{-}^{2}\right) & \mapsto w^{+},
\end{aligned}
$$

be the linear mappings defined as follows. Let $\left(z^{1}, z^{2}\right) \in \mathcal{C}\left(\left[\tau_{0}, \tau_{4}^{\lambda}+\tau^{\prime}\right] \times[0,1]\right)^{2}$ be the solution of

$$
\begin{gathered}
z_{t}^{1}+\lambda_{1} z_{x}^{1}=z_{t}^{2}+\lambda_{2} z_{x}^{2}=0 \\
\left(1+\frac{\gamma}{4}\right)\left(z^{2}(t, 0)-z^{1}(t, 0)\right)=(1+\gamma)\left(z^{2}(t, 1)-z^{1}(t, 1)\right), \\
z^{2}(t, 0)=0 \forall t \in\left[\tau_{0}, \tau_{4}^{\lambda}\right] \\
z^{2}(t, 0)-z^{1}(t, 0)=-\kappa\left(t-\tau_{4}^{\lambda}\right) z^{1}\left(\tau_{4}^{\lambda}, 0\right) \forall t \in\left[\tau_{4}^{\lambda}, \tau_{4}^{\lambda}+\tau^{\prime}\right], \\
z^{1}\left(\tau_{0}, \cdot\right)=\alpha_{-}^{1}, z^{2}\left(\tau_{0}, \cdot\right)=\alpha_{-}^{2} .
\end{gathered}
$$

It is not hard to see that there exists one and only one such a $\left(z^{1}, z^{2}\right)$, at least if $\nu$ and $\gamma$ are small enough. Let $\left(\alpha_{+}^{1}, \alpha_{+}^{2}\right) \in C^{0}([0,1])^{2}$ and $w^{+} \in C^{0}\left(\left[0, T^{*}\right]\right)$ be defined by

$$
\begin{gathered}
\left(\alpha_{+}^{1}(x), \alpha_{+}^{2}(x)\right):=\left(z^{1}\left(\tau_{4}^{\lambda}+\tau^{\prime}, x\right), z^{2}\left(\tau_{4}^{\lambda}+\tau^{\prime}, x\right)\right), \\
2 w^{+}(t):=\left(1+\frac{\gamma}{4}\right)\left(z^{2}(t, 0)-z^{1}(t, 0)\right) \quad\left(=(1+\gamma)\left(z^{2}(t, 1)-z^{1}(t, 1)\right)\right) .
\end{gathered}
$$

Note that, by (A.10, A.12, A.14, A.16) and (A.17),

$$
w^{+}\left(\tau_{4}^{\lambda}+\tau^{\prime}\right)=0,\left(\alpha_{+}^{1}, \alpha_{+}^{2}\right) \in \mathcal{A}^{+} .
$$

Similarly, let

$$
\begin{aligned}
\mathbf{P}_{\lambda, \gamma}^{-}: \mathcal{A}^{+} & \rightarrow \mathcal{C}^{0}([0,1])^{2} \\
\left(\beta_{+}^{1}, \beta_{+}^{2}\right) & \mapsto\left(\beta_{-}^{1}, \beta_{-}^{2}\right),
\end{aligned}
$$

and

$$
\begin{aligned}
\mathbf{W}_{\lambda, \gamma}^{-}: \mathcal{A}^{+} & \rightarrow \mathcal{C}^{0}\left(\left[\tau_{0}, \tau_{4}^{\lambda}+\tau^{\prime}\right]\right) \\
\left(\beta_{+}^{1}, \beta_{+}^{2}\right) & \mapsto w^{-},
\end{aligned}
$$


be the linear mappings defined as follows. Let $\left(y^{1}, y^{2}\right) \in \mathcal{C}\left(\left[\tau_{0}, \tau_{4}^{\lambda}+\tau^{\prime}\right] \times[0,1]\right)^{2}$ be such that

$$
\begin{gathered}
y_{t}^{1}+\lambda_{1} y_{x}^{1}=y_{t}^{2}+\lambda_{2} y_{x}^{2}=0, \\
\left(1+\frac{\gamma}{4}\right)\left(y^{2}(t, 0)-y^{1}(t, 0)\right)=(1+\gamma)\left(y^{2}(t, 1)-y^{1}(t, 1)\right), \\
y^{2}(t, 0)-y^{1}(t, 0)=0 \forall t \in\left[\tau_{0}+\tau^{\prime}, \tau_{4}^{\lambda}+\tau^{\prime}\right], \\
\left(1+\frac{\gamma}{4}\right)\left(y^{2}(t, 0)-y^{1}(t, 0)\right)=-(1+\gamma) \kappa\left(t-\tau_{0}\right) y^{1}\left(\tau_{4}^{\lambda}, 1\right) \forall t \in\left[\tau_{0}, \tau_{0}+\tau^{\prime}\right], \\
y^{1}\left(\tau_{4}^{\lambda}+\tau^{\prime}, \cdot\right)=\alpha_{+}^{1}, y^{2}\left(\tau_{4}^{\lambda}+\tau^{\prime}, \cdot\right)=\alpha_{+}^{2} .
\end{gathered}
$$

It is not hard to see that there exists one and only one such a $\left(y^{1}, y^{2}\right)$, at least if $\nu>0$ and $\gamma>0$ are small enough - which is always assumed. We let

$$
\begin{gathered}
\left(\beta_{-}^{1}(x), \beta_{-}^{2}(x)\right):=\left(y^{1}\left(\tau_{0}, x\right), y^{2}\left(\tau_{0}, x\right)\right) \\
2 w^{-}(t):=\left(1+\frac{\gamma}{4}\right)\left(y^{2}(t, 0)-y^{1}(t, 0)\right) \quad\left(=(1+\gamma)\left(y^{2}(t, 1)-y^{1}(t, 1)\right)\right) .
\end{gathered}
$$

Let us point out that

$$
\mathbf{P}_{\lambda, \gamma}^{-} \mathbf{P}_{\lambda, \gamma}^{+}\left(\mathcal{A}_{\gamma}^{-}\right) \subset \mathcal{A}_{\gamma}^{-} .
$$

Indeed, if with the above notations we have $\left(\beta_{+}^{1}, \beta_{+}^{2}\right)=\left(\alpha_{+}^{1}, \alpha_{+}^{2}\right)$, then one has, following the characteristic curves and using the boundary conditions for $\left(y^{1}, y^{2}\right)$ and $\left(z^{1}, z^{2}\right)$,

$$
y^{2}\left(\tau_{0}, 0\right)=y^{2}\left(\tau_{1}^{\lambda}, 1\right)=y^{1}\left(\tau_{1}^{\lambda}, 1\right)=y^{1}\left(\tau_{4}^{\lambda}, 0\right)=y^{2}\left(\tau_{4}^{\lambda}, 0\right)=\beta_{+}^{2}\left(a_{\lambda}\right)=\alpha_{+}^{2}\left(a_{\lambda}\right)=z^{2}\left(\tau_{4}^{\lambda}, 0\right)=0,
$$

where $a_{\lambda}$ is defined by

$$
\left(\dot{\xi}=\lambda_{2}(t, \xi) \text { and } \xi\left(\tau_{4}^{\lambda}\right)=0\right) \Rightarrow\left(\xi\left(\tau_{4}^{\lambda}+\tau^{\prime}\right)=a_{\lambda}\right) .
$$

Let us assume for the moment being that the following lemma holds:

Lemma 25. There exists $C_{28}>1, \nu_{0}>0$ and $\gamma_{13} \in(0,1 / 2]$ such that, for every $\nu \in\left(0, \nu_{0}\right]$, for every $\lambda \in \mathcal{L}_{\nu, \gamma}^{0}$, for every $\gamma \in\left(0, \gamma_{13}\right]$, for every $\alpha \in \mathcal{A}_{\gamma}^{-}$, for every $\beta \in \mathcal{A}^{+}$, and for every $\rho \in[0, \infty)$,

$$
\begin{gathered}
\left|\mathbf{P}_{\lambda, \gamma}^{+} \alpha\right|_{0} \leqslant C_{28}|\alpha|_{0}, \\
\omega_{\rho}\left(\mathbf{P}_{\lambda, \gamma}^{+} \alpha\right) \leqslant C_{28}\left(\omega_{\rho}(\alpha)+\nu \rho|\alpha|_{0}\right), \\
\left|\mathbf{P}_{\lambda, \gamma}^{-} \mathbf{P}_{\lambda, \gamma}^{+} \alpha\right|_{0} \leqslant\left(1-\frac{11 \gamma}{16}+C_{28} \nu\right)|\alpha|_{0}, \\
\omega_{\rho}\left(\mathbf{P}_{\lambda, \gamma}^{-} \mathbf{P}_{\lambda, \gamma}^{+} \alpha\right) \leqslant\left(1-\frac{11 \gamma}{16}+C_{28} \nu\right) \omega_{\left(1+\frac{5 \gamma}{8}+C_{28} \nu\right) \rho} \omega_{\rho}(\alpha)+C_{28} \nu \rho|\alpha|_{0}, \\
\left|\mathbf{W}_{\lambda, \gamma}^{+}(\alpha)\right|_{0} \leqslant C_{28}|\alpha|_{0}, \\
\omega_{\rho}\left(\mathbf{W}_{\lambda, \gamma}^{+}(\alpha)\right) \leqslant C_{28}\left(\omega_{\rho}(\alpha)+\nu \rho|\alpha|_{0}\right), \\
\left|\mathbf{W}_{\lambda, \gamma}^{-}(\alpha)\right|_{0} \leqslant C_{28}|\alpha|_{0}, \\
\omega_{\rho}\left(\mathbf{W}_{\lambda, \gamma}^{-}(\beta)\right) \leqslant C_{28}\left(\omega_{\rho}(\beta)+\nu \rho|\beta|_{0}\right) .
\end{gathered}
$$

Let

$$
\gamma_{12}:=\operatorname{Min}\left(\frac{1}{64 C_{28}}, \sqrt{\nu_{0}}, \gamma_{13}\right)
$$


Let us then prove that Lemma 24 holds with $C_{27}$ large enough and

$$
\begin{gathered}
\mathbf{W}_{\gamma}^{0}(\lambda, \alpha)=\left(\sum_{i=0}^{+\infty} \mathbf{W}_{\lambda, \gamma}^{+}\left(\mathbf{P}_{\lambda, \gamma}^{-} \mathbf{P}_{\lambda, \gamma}^{+}\right)^{i}-\mathbf{W}_{\lambda, \gamma}^{-} \mathbf{P}_{\lambda, \gamma}^{+}\left(\mathbf{P}_{\lambda, \gamma}^{-} \mathbf{P}_{\lambda, \gamma}^{+}\right)^{i}\right) \alpha \text { on }\left[\tau_{0}, \tau_{4}^{\lambda}+\tau^{\prime}\right] \\
\mathbf{W}_{\gamma}^{0}(\lambda, \alpha)=0 \text { on }\left[\tau_{4}^{\lambda}+\tau^{\prime}, T^{*}\right]
\end{gathered}
$$

Indeed, let $\gamma \in\left(0, \gamma_{12}\right], \lambda=\left(\lambda^{1}, \lambda^{2}\right) \in \mathcal{L}_{\gamma_{12} \gamma, \gamma}^{0}$ and $\alpha \in \mathcal{A}_{\gamma}^{-}$. From (A.25) and (A.33), one gets, for every $i \in \mathbb{N}$,

$$
\left|\left(\mathbf{P}_{\lambda, \gamma}^{-} \mathbf{P}_{\lambda, \gamma}^{+}\right)^{i} \alpha\right| \leqslant\left(1-\frac{43 \gamma}{64}\right)^{i}|\alpha|_{0}
$$

From (A.18, A.25, A.29, A.31, A.34) and (A.35), one gets the existence and the continuity of $\mathbf{W}_{\gamma}^{0}(\lambda, \alpha)$, as well as (A.8) provided that

$$
C_{27} \geqslant \frac{64 C_{28}}{43}\left(1+C_{28}\right)
$$

Moreover $\mathbf{W}_{\gamma}^{0}$ is also continuous from $\mathcal{L}_{\gamma_{12} \gamma, \gamma}^{0} \times \mathcal{A}_{\gamma}^{-}$into $C^{0}\left(\left[\tau_{0}, T^{*}\right]\right)$ and $\mathbf{W}_{\gamma}^{0}(\lambda, \cdot)$ is linear. Let us check that (A.9) holds for $C_{27}$ large enough. Let again $\gamma \in\left(0, \gamma_{12}\right], \lambda=\left(\lambda^{1}, \lambda^{2}\right) \in \mathcal{L}_{\gamma_{12} \gamma, \gamma}^{0}$ and $\alpha \in \mathcal{A}_{\gamma}^{-}$. From (A.27, A.28) and (A.33), one gets, for every $\rho \in[0,+\infty)$ and for every $i \in \mathbb{N} \backslash\{0\}$,

$$
\begin{aligned}
\omega_{\rho}\left(\left(\mathbf{P}_{\lambda, \gamma}^{-} \mathbf{P}_{\lambda, \gamma}^{+}\right)^{i} \alpha\right) & \leqslant\left(1-\frac{43 \gamma}{64}\right)^{i} \omega_{\left(1+\frac{41 \gamma}{64}\right)^{i} \rho}(\alpha)+\frac{\gamma}{64}\left(1-\frac{43 \gamma}{64}\right)^{i-1} \sum_{j=0}^{i-1}\left(1+\frac{41 \gamma}{64}\right)^{j} \rho|\alpha|_{0} \\
& \leqslant\left(1-\frac{43 \gamma}{64}\right)^{i} \omega_{\left(1+\frac{41 \gamma}{64}\right)^{i} \rho}(\alpha)+\frac{1}{41}\left(1+\frac{41 \gamma}{64}\right)\left(1-\frac{\gamma}{32}\right)^{i-1} \rho|\alpha|_{0} .
\end{aligned}
$$

Let us point out that, for every compact convex subset $K$ of $\mathbb{R}^{n}$, for every continuous map $f: K \rightarrow \mathbb{R}^{m}$ and for every real numbers $C \geqslant 1$ and $\rho \geqslant 0$,

$$
\omega_{C \rho}(f) \leqslant([C]+1) \omega_{\rho}(f) \leqslant 2 C \omega_{\rho}(f)
$$

where $[C]$ denotes the integer part of $C$. Hence, from (A.37), one gets (let us recall that $C_{28}>1$ and that $\gamma \in(0,1])$

$$
\omega_{\rho}\left(\left(\mathbf{P}_{\lambda, \gamma}^{-} \mathbf{P}_{\lambda, \gamma}^{+}\right)^{i} \alpha\right) \leqslant 2 C_{28}\left(1-\frac{\gamma}{32}\right)^{i}\left(\omega_{\rho}(\alpha)+\rho|\alpha|_{0}\right) \forall \rho \in[0,+\infty), \forall i \in \mathbb{N}
$$

From (A.25, A.26, A.30, A.32, A.34, A.36) and (A.38), one gets (A.9) provided that $C_{27}$ is large enough.

Finally it remains to check that (i) of Lemma 24 holds. Let $\gamma \in\left(0, \gamma_{12}\right], \lambda=\left(\lambda^{1}, \lambda^{2}\right) \in \mathcal{L}_{\gamma_{12} \gamma, \gamma}^{0}$ and $\alpha \in \mathcal{A}_{\gamma}^{-}$. For $\bar{\alpha} \in \mathcal{A}_{\gamma}^{-}$and $\bar{w} \in \mathcal{C}^{0}\left(\left[\tau_{0}, \tau_{4}^{\lambda}+\tau^{\prime}\right]\right.$ satisfying

$$
2 \bar{w}(0)=\left(1+\frac{\gamma}{4}\right)\left(\bar{\alpha}^{2}(0)-\bar{\alpha}^{1}(0)\right)=(1+\gamma)\left(\bar{\alpha}^{2}(1)-\bar{\alpha}^{1}(1)\right),
$$

let us define

$$
\Phi_{\lambda, \gamma}(\bar{w}, \bar{\alpha}):=\left(\bar{z}^{1}\left(\tau_{4}^{\lambda}+\tau^{\prime}, \cdot\right), \bar{z}^{2}\left(\tau_{4}^{\lambda}+\tau^{\prime}, \cdot\right)\right) \in \mathcal{C}^{0}([0,1])^{2}
$$


where $\left(\bar{z}^{1}, \bar{z}^{2}\right) \in \mathcal{C}^{0}\left(\left[\tau_{0}, \tau_{4}^{\lambda}+\tau^{\prime}\right] \times[0,1]\right)^{2}$ are such that

$$
\begin{gathered}
\bar{z}_{t}^{1}+\lambda_{1} \bar{z}_{x}^{1}=\bar{z}_{t}^{2}+\lambda_{2} \bar{z}_{x}^{2}=0, \\
\left(1+\frac{\gamma}{4}\right)\left(\bar{z}^{2}(t, 0)-\bar{z}^{1}(t, 0)\right)=(1+\gamma)\left(\bar{z}^{2}(t, 1)-\bar{z}^{1}(t, 1)\right)=2 \bar{w}(t), \\
\bar{z}^{1}\left(\tau_{0}, \cdot\right)=\alpha^{1}, \bar{z}^{2}\left(\tau_{0}, \cdot\right)=\alpha^{2} .
\end{gathered}
$$

Then, for every $\beta \in \mathbf{P}_{\lambda, \gamma}^{+}\left(\mathcal{A}_{\gamma}^{-}\right)$

$$
\Phi_{\lambda, \gamma}\left(\mathbf{W}_{\lambda, \gamma}^{+} \mathbf{P}_{\lambda, \gamma}^{-} \beta-\mathbf{W}_{\lambda, \gamma}^{-} \beta, 0\right)=\Phi_{\lambda, \gamma}\left(\mathbf{W}_{\lambda, \gamma}^{+} \mathbf{P}_{\lambda, \gamma}^{-} \beta, \mathbf{P}_{\lambda, \gamma}^{-} \beta\right)-\Phi_{\lambda, \gamma}\left(\mathbf{W}_{\lambda, \gamma}^{-} \beta, \mathbf{P}_{\lambda, \gamma}^{-} \beta\right)=\mathbf{P}_{\lambda, \gamma}^{+} \mathbf{P}_{\lambda, \gamma}^{-} \beta-\beta .
$$

Hence, for every $i \in \mathbb{N} \backslash\{0\}$,

$$
\begin{aligned}
\Phi_{\lambda, \gamma}\left(\mathbf{W}_{\lambda, \gamma}^{+}\left(\mathbf{P}_{\lambda, \gamma}^{-} \mathbf{P}_{\lambda, \gamma}^{+}\right)^{i} \alpha-\mathbf{W}_{\lambda, \gamma}^{-} \mathbf{P}_{\lambda, \gamma}^{+}\left(\mathbf{P}_{\lambda, \gamma}^{-} \mathbf{P}_{\lambda, \gamma}^{+}\right)^{i-1} \alpha, 0\right) & =\mathbf{P}_{\lambda, \gamma}^{+}\left(\mathbf{P}_{\lambda, \gamma}^{-} \mathbf{P}_{\lambda, \gamma}^{+}\right)^{i} \alpha-\mathbf{P}_{\lambda, \gamma}^{+}\left(\mathbf{P}_{\lambda, \gamma}^{-} \mathbf{P}_{\lambda, \gamma}^{+}\right)^{i-1} \alpha
\end{aligned}
$$

Since

we get, with (A.34) and (A.39),

$$
\Phi_{\lambda, \gamma}\left(\mathbf{W}_{\lambda, \gamma}^{+} \alpha, \alpha\right)=\mathbf{P}_{\lambda, \gamma}^{+} \alpha
$$

$$
\Phi_{\lambda, \gamma}\left(\mathbf{W}_{\gamma}(\lambda, \alpha), \alpha\right)=0
$$

Let $z=\left(z^{1}, z^{2}\right) \in \mathcal{C}^{0}\left(\left[\tau_{0}, T^{*}\right] \times[0,1]\right)^{2}$ be such that (A.2, A.3) and (A.4) hold with $w=\mathbf{W}^{0}(\lambda, \alpha)$. It follows from (A.40) that (A.6) holds. Let

$$
e(t)=\int_{0}^{1}\left(z^{1}+z^{2}\right)(t, x) \mathrm{d} x
$$

Note that by (A.5)

$$
e\left(T^{*}\right)=0
$$

Moreover, by (A.8), there exists $C_{29}>0$, independent of $\gamma \in\left(0, \gamma_{12}\right]$ and of $(\lambda, \alpha) \in \mathcal{L}_{\gamma_{12} \gamma, \gamma}^{0}$ such that

$$
\left|z^{1}\right|_{0}+\left|z^{2}\right|_{0} \leqslant \frac{C_{29}}{\gamma}|\alpha|_{0}
$$

Taking the derivative with respect to time of (A.41), one gets, with (A.2, A.3) and an integration by parts,

$$
\begin{aligned}
\dot{e}(t)= & \left(1+\gamma-\lambda_{2}(t, 1)\right) z^{2}(t, 1)-\left(1+\gamma+\lambda_{1}(t, 1)\right) z^{1}(t, 1)-\left(1+\frac{\gamma}{4}-\lambda_{2}(t, 0)\right) z^{2}(t, 0) \\
& +\left(1+\frac{\gamma}{4}+\lambda_{1}(t, 0)\right) z^{1}(t, 0)+\int_{0}^{1}\left(\lambda_{1 x} z^{1}+\lambda_{2 x} z^{2}\right)(t, x) \mathrm{d} x .
\end{aligned}
$$

Increasing if necessary $C_{27}$, one gets (A.6) and (A.7) from (A.41-A.44) and Gronwall's lemma.

Let us now prove Lemma 25. The estimates (A.25) to (A.32) are obtained by giving explicit expressions of the linear operators $\mathbf{P}_{\lambda, \gamma}^{+}, \mathbf{P}_{\lambda, \gamma}^{-}, \mathbf{W}_{\lambda, \gamma}^{+}$and $\mathbf{W}_{\lambda, \gamma}^{-}$. For simplicity we shall give only the proofs of the two more complicate estimates, which are (A.27) and (A.28). Let $\nu>0, \gamma \in[0,1], \lambda \in \mathcal{L}_{\nu, \gamma}^{0}$ and $\alpha_{-}=\left(\alpha_{-}^{1}, \alpha_{-}^{2}\right) \in \mathcal{A}_{\gamma}^{-}$. 
Let $\left(z^{1}, z^{2}\right) \in \mathcal{C}^{0}\left(\left[\tau_{0}, \tau_{4}^{\lambda}+\tau^{\prime}\right] \times[0,1]\right)^{2}$ and $\left(y^{1}, y^{2}\right) \in \mathcal{C}^{0}\left(\left[\tau_{0}, \tau_{4}^{\lambda}+\tau^{\prime}\right] \times[0,1]\right)^{2}$ be such that (A.11) to (A.15, A.19) to (A.22) hold and

$$
y\left(\tau_{4}^{\lambda}+\tau^{\prime}, \cdot\right)=z\left(\tau_{4}^{\lambda}+\tau^{\prime}, \cdot\right) .
$$

Let us first an explicit expression for $z\left(\tau_{4}^{\lambda}, \cdot\right)$. One has

$$
\begin{gathered}
z^{2}\left(\tau_{4}^{\lambda}, x\right)=0 \\
z^{1}\left(\tau_{4}^{\lambda}, x\right)=\left(\frac{1+\frac{\gamma}{4}}{1+\gamma}\right)^{2} \alpha_{-}^{1}\left(p_{1}(x)\right)+\frac{1+\frac{\gamma}{4}}{1+\gamma} \alpha_{-}^{2}\left(p_{2}(x)\right),
\end{gathered}
$$

where $p_{1}(x)$ and $p_{2}(x)$ are the real numbers in $[0,1]$ such that there exist $t_{2}^{-}(x) \in\left[\tau_{3}^{\lambda}, \tau_{4}^{\lambda}\right]$ and $t_{1}^{-}(x) \in\left[\tau_{0}, \tau_{3}^{\lambda}\right]$ satisfying

$$
\begin{gathered}
\left(\dot{\xi}=\lambda_{1}(t, \xi) \text { and } \xi\left(\tau_{4}^{\lambda}\right)=x\right) \Rightarrow\left(\xi\left(t_{2}^{-}(x)\right)=1\right), \\
\left(\dot{\xi}=\lambda_{1}(t, \xi) \text { and } \xi\left(t_{2}^{-}(x)\right)=0\right) \Rightarrow\left(\xi\left(t_{1}^{-}(x)\right)=1\right), \\
\left(\dot{\xi}=\lambda_{1}(t, \xi) \text { and } \xi\left(t_{1}^{-}(x)\right)=0\right) \Rightarrow\left(\xi\left(\tau_{0}\right)=p_{1}(x)\right), \\
\left(\dot{\xi}=\lambda_{2}(t, \xi) \text { and } \xi\left(t_{1}^{-}(x)\right)=1\right) \Rightarrow\left(\xi\left(\tau_{0}\right)=p_{2}(x)\right) .
\end{gathered}
$$

Of course, $p_{1}, p_{2}, t_{1}^{-}$and $t_{2}^{-}$depend on $\lambda$ so we should write for example $p_{1}^{\lambda}, p_{2}^{\lambda}, t_{1}^{\lambda,-}$ and $t_{2}^{\lambda,-}$. For simplicity we omit the index $\lambda$

Let us also define $\psi=\left(\psi^{1}, \psi^{2}\right) \in \mathcal{C}^{0}\left(\left[\tau_{4}^{\lambda}, \tau_{4}^{\lambda}+\tau^{\prime}\right] \times[0,1]\right)^{2}$ by requiring

$$
\begin{gathered}
\psi_{t}^{1}+\lambda_{1} \psi_{x}^{1}=\psi_{t}^{2}+\lambda_{2} \psi_{x}^{2}=0 \\
\psi^{1}\left(\tau_{4}^{\lambda}+\tau^{\prime}, \cdot\right)=0, \psi^{2}\left(\tau_{4}^{\lambda}+\tau^{\prime}, \cdot\right)=0 \\
\left(1+\frac{\gamma}{4}\right)\left(\psi^{2}(t, 0)-\psi^{1}(t, 0)\right)=(1+\gamma)\left(\psi^{2}(t, 1)-\psi^{1}(t, 1)\right)=\kappa\left(t-\tau_{4}^{\lambda}\right) .
\end{gathered}
$$

Of course, $\psi$ depends on $\lambda$ and $\gamma$. So we should write, for example, $\psi^{\lambda, \gamma}$. Again for simplicity we omit the indices $\lambda$ and $\gamma$. We use this simplification until the end of the proof of Lemma 25. So we write, for example, $\tau_{i}$ for $\tau_{i}^{\lambda}$. It follows from (A.10-A.12, A.14, A.19-A.21, A.45, A.48, A.49) and (A.50) that

$$
y(t, x)=z(t, x)+\left(1+\frac{\gamma}{4}\right) z^{1}\left(\tau_{4}, 0\right) \psi(t, x) \forall(t, x) \in\left[\tau_{4}, \tau_{4}+\tau^{\prime}\right] \times[0,1] .
$$

In particular,

$$
y\left(\tau_{4}, \cdot\right)=z\left(\tau_{4}, \cdot\right)+\left(1+\frac{\gamma}{4}\right) z^{1}\left(\tau_{4}, 0\right) \psi\left(\tau_{4}, \cdot\right) .
$$

Let $\left(\bar{y}^{1}, \bar{y}^{2}\right) \in \mathcal{C}^{0}\left(\left[\tau_{0}, \tau_{4}\right] \times[0,1]\right)^{2}$ be such that

$$
\begin{gathered}
\bar{y}_{t}^{1}+\lambda_{1} \bar{y}_{x}^{1}=\bar{y}_{t}^{2}+\lambda_{2} \bar{y}_{x}^{2}=0, \\
\left(\bar{y}^{2}(t, 0)-\bar{y}^{1}(t, 0)\right)=\left(\bar{y}^{2}(t, 1)-\bar{y}^{1}(t, 1)\right)=0, \\
\bar{y}^{1}\left(\tau_{4}, \cdot\right)=y^{1}\left(\tau_{4}, \cdot\right), \bar{y}^{2}\left(\tau_{4}, \cdot\right)=y^{2}\left(\tau_{4}, \cdot\right) .
\end{gathered}
$$


Note that, by (A.19) to (A.21) and (A.52) to (A.54),

$$
\bar{y}=y \text { on }\left[\tau_{0}+\tau^{\prime}, \tau_{4}\right] \times[0,1] .
$$

Following the characteristic curves $\dot{\xi}=\lambda_{1}(t, \xi), \dot{\xi}=\lambda_{2}(t, \xi)$ and using the boundary conditions (A.53, A.54), one gets

$$
\bar{y}^{1}\left(\tau_{0}, x\right)=y^{1}\left(\tau_{4}, q_{1}(x)\right), \bar{y}^{2}\left(\tau_{0}, x\right)=y^{2}\left(\tau_{4}, q_{2}(x)\right),
$$

where $q_{1}(x)$ and $q_{2}(x)$ are the real numbers in $[0,1]$ such that there exist $\left(t_{1}(x), t_{3}(x)\right) \in\left[\tau_{0}, \tau_{1}\right]^{2}$ and $\left(t_{2}(x), t_{4}(x)\right) \in\left[\tau_{1}, \tau_{4}\right]^{2}$ satisfying

$$
\begin{gathered}
\left(\dot{\xi}=\lambda_{2}(t, \xi) \text { and } \xi\left(\tau_{0}\right)=x\right) \Rightarrow\left(\xi\left(t_{1}(x)\right)=1\right), \\
\left(\dot{\xi}=\lambda_{1}(t, \xi) \text { and } \xi\left(t_{1}(x)\right)=1\right) \Rightarrow\left(\xi\left(t_{2}(x)\right)=0\right), \\
\left(\dot{\xi}=\lambda_{1}(t, \xi) \text { and } \xi\left(\tau_{0}\right)=x\right) \Rightarrow\left(\xi\left(t_{3}(x)\right)=0\right), \\
\left(\dot{\xi}=\lambda_{2}(t, \xi) \text { and } \xi\left(t_{3}(x)\right)=0\right) \Rightarrow\left(\xi\left(t_{4}(x)\right)=1\right), \\
\left(\dot{\xi}=\lambda_{2}(t, \xi) \text { and } \xi\left(t_{2}(x)\right)=0\right) \Rightarrow\left(q_{2}(x)=\xi\left(\tau_{4}\right)\right), \\
\left(\dot{\xi}=\lambda_{1}(t, \xi) \text { and } \xi\left(t_{4}(x)\right)=1\right) \Rightarrow\left(q_{1}(x)=\xi\left(\tau_{4}\right)\right) .
\end{gathered}
$$

Let us now define $\phi=\left(\phi^{1}, \phi^{2}\right) \in \mathcal{C}^{0}\left(\left[\tau_{0}, \tau_{0}+\tau^{\prime}\right] \times[0,1]\right)^{2}$ by requiring

$$
\begin{gathered}
\phi_{t}^{1}+\lambda_{1} \phi_{x}^{1}=\phi_{t}^{2}+\lambda_{2} \phi_{x}^{2}=0, \\
\phi^{1}\left(\tau_{0}+\tau^{\prime}, \cdot\right)=0, \phi^{2}\left(\tau_{0}+\tau^{\prime}, \cdot\right)=0, \\
\left(1+\frac{\gamma}{4}\right)\left(\phi^{2}(t, 0)-\phi^{1}(t, 0)\right)=(1+\gamma)\left(\phi^{2}(t, 1)-\phi^{1}(t, 1)\right)=\kappa\left(t-\tau_{0}\right) \forall t \in\left[\tau_{0}, \tau_{0}+\tau^{\prime}\right] .
\end{gathered}
$$

It follows from (A.19, A.20, A.22, A.52, A.53, A.55, A.57, A.58) and (A.59) that

$$
y\left(\tau_{0}, \cdot\right)=\bar{y}\left(\tau_{0}, \cdot\right)-(1+\gamma) y^{1}\left(\tau_{4}, 1\right) \phi\left(\tau_{0}, \cdot\right) .
$$

Note that, by (A.48) and (A.49), one has, at least if $\gamma$ and $\nu$ are small enough

$$
\psi^{1}\left(\tau_{4}, 1\right)=0,
$$

which, with (A.51), gives

$$
y^{1}\left(\tau_{4}, 1\right)=z^{1}\left(\tau_{4}, 1\right)
$$

From (A.47), one gets

$$
\begin{aligned}
z^{1}\left(\tau_{4}, 1\right)=\left(\frac{1+\frac{\gamma}{4}}{1+\gamma}\right)^{2} \alpha_{-}^{1}\left(p_{1}(1)\right)+\frac{1+\frac{\gamma}{4}}{1+\gamma} \alpha_{-}^{2}\left(p_{2}(1)\right) & =\left(\frac{1+\frac{\gamma}{4}}{1+\gamma}\right)^{2} \alpha_{-}^{1}(1)+\frac{1+\frac{\gamma}{4}}{1+\gamma} \alpha_{-}^{2}(0) \\
& =\left(\frac{1+\frac{\gamma}{4}}{1+\gamma}\right)^{2} \alpha_{-}^{1}(1)
\end{aligned}
$$


which, with (A.60) and (A.61), gives

$$
y\left(\tau_{0}, \cdot\right)=\bar{y}\left(\tau_{0}, \cdot\right)-\frac{\left(1+\frac{\gamma}{4}\right)^{2}}{1+\gamma} \alpha_{-}^{1}(1) \phi\left(\tau_{0}, \cdot\right) .
$$

From (A.12, A.46) and (A.62), one gets

$$
z^{1}\left(\tau_{4}, 0\right)=\frac{1+\frac{\gamma}{4}}{1+\gamma} \alpha_{-}^{1}(1)
$$

From (A.46, A.47, A.51, A.56, A.60, A.63) and (A.64) one gets

$$
\begin{aligned}
y^{1}\left(\tau_{0}, x\right)= & \left(\frac{1+\frac{\gamma}{4}}{1+\gamma}\right)^{2} \alpha_{-}^{1}\left(p_{1} \circ q_{1}(x)\right)+\frac{1+\frac{\gamma}{4}}{1+\gamma} \alpha_{-}^{2}\left(p_{2} \circ q_{1}(x)\right) \\
& +\frac{\left(1+\frac{\gamma}{4}\right)^{2}}{1+\gamma} \alpha_{-}^{1}(1)\left(\psi^{1}\left(\tau_{4}, q_{1}(x)\right)-\phi^{1}\left(\tau_{0}, x\right)\right), \\
y^{2}\left(\tau_{0}, x\right)= & \frac{\left(1+\frac{\gamma}{4}\right)^{2}}{1+\gamma} \alpha_{-}^{1}(1)\left(\psi^{2}\left(\tau_{4}, q_{2}(x)\right)-\phi^{2}\left(\tau_{0}, x\right)\right) .
\end{aligned}
$$

Let us assume for the moment being that $\lambda=\left(-\sqrt{H^{\gamma}}, \sqrt{H^{\gamma}}\right)$ with $\lambda$ small enough so that $\lambda \in \mathcal{L}^{0}$. Then, clearly,

$$
\phi\left(\tau_{0}+t, \cdot\right)=\psi\left(\tau_{4}+t, \cdot\right) \forall t \in\left[0, \tau^{\prime}\right]
$$

and straightforward computations lead to

$$
\begin{gathered}
q_{1}(x)=q_{2}(x)=p_{1}(x)=x \forall x \in[0,1], \\
\sqrt{1+\gamma\left(\frac{1}{2}-p_{2} \circ q_{1}(x)\right)}=\sqrt{1+\frac{\gamma}{2}}+\sqrt{1-\frac{\gamma}{2}}-\sqrt{1+\gamma\left(\frac{1}{2}-x\right)} \forall x \in[0,1] .
\end{gathered}
$$

Differentiating (A.69) with respect to $x$ one gets

$$
\left|\left(p_{2} \circ q_{1}\right)^{\prime}\right|_{0}=\sqrt{\frac{1+\frac{\gamma}{2}}{1-\frac{\gamma}{2}}} .
$$

From (A.67, A.68, A.70) and straightforward estimates, one gets the existence of $C_{30}>1, \nu_{1}>0$ and $\gamma_{14}$ $\in(0,1 / 2]$ such that, for every $\nu \in\left(0, \nu_{1}\right]$, for every $\gamma \in\left(0, \gamma_{14}\right]$ and for every $\lambda \in \mathcal{L}_{\nu, \gamma}^{0}$

$$
\begin{gathered}
\left|\psi\left(\tau_{4}, q_{1}(\cdot)\right)-\phi\left(\tau_{0}, \cdot\right)\right|_{1}+\left|\psi\left(\tau_{4}, q_{2}(\cdot)\right)-\phi\left(\tau_{0}, \cdot\right)\right|_{1} \leqslant C_{30} \nu, \\
\left|\left(p_{1} \circ q_{1}\right)^{\prime}\right|_{0} \leqslant 1+C_{30} \nu, \\
\left|\left(p_{2} \circ q_{1}\right)^{\prime}\right|_{0} \leqslant 1+\frac{5 \gamma}{8}+C_{30} \nu .
\end{gathered}
$$

These inequalities, together with (A.65) and (A.66), give (A.27) and (A.28) provided that $C_{28}$ is large enough and $\gamma_{13}>0$ is small enough (let us recall that $|\alpha|_{0}=\left|\alpha^{1}\right|_{0}+\left|\alpha^{2}\right|_{0}$ and that $\omega_{\rho}(\alpha)=\omega_{\rho}\left(\alpha^{1}\right)+\omega_{\rho}\left(\alpha^{2}\right)$ (see (3.8) and (4.49))). 
Remark 26. The proof of Lemma 25 relies heavily on the fact that $\theta(\lambda)=0$. Indeed, let us for example study the case where $\tau_{3}^{\lambda}<\tau_{1}^{\lambda}$. Let $t_{0} \in\left(\tau_{3}^{\lambda}, \tau_{1}^{\lambda}\right)$. Let us define $\left(x_{0}, x_{1}, x_{2}, x_{3}\right) \in(0,1)^{4}, t_{1} \in\left(T^{*} / 2, \tau_{3}^{\lambda}\right), t_{2} \in\left(0, \tau_{1}^{\lambda}\right)$ by requiring

$$
\begin{gathered}
\left(\dot{\xi}=\lambda_{1}(t, \xi) \text { and } \xi\left(t_{0}\right)=1\right) \Rightarrow\left(\xi\left(t_{1}\right)=0\right), \\
\left(\dot{\xi}=\lambda_{1}(t, \xi) \text { and } \xi\left(t_{1}\right)=1\right) \Rightarrow\left(\xi\left(\tau_{4}^{\lambda}\right)=x_{0}\right), \\
\left(\dot{\xi}=\lambda_{1}(t, \xi) \text { and } \xi\left(t_{0}\right)=0\right) \Rightarrow\left(\xi\left(t_{2}\right)=1\right), \\
\left(\dot{\xi}=\lambda_{1}(t, \xi) \text { and } \xi\left(t_{2}\right)=0\right) \Rightarrow\left(\xi\left(\tau_{0}\right)=x_{1}\right), \\
\left(\dot{\xi}=\lambda_{2}(t, \xi) \text { and } \xi\left(t_{0}\right)=1\right) \Rightarrow\left(\xi\left(\tau_{0}\right)=x_{2}\right) \\
\left(\dot{\xi}=\lambda_{2}(t, \xi) \text { and } \xi\left(t_{2}\right)=1\right) \Rightarrow\left(\xi\left(\tau_{0}\right)=x_{3}\right)
\end{gathered}
$$

For $\nu>0$ and $\gamma>0$ small enough such $\left(x_{0}, x_{1}, x_{2}, x_{3}\right), t_{1}, t_{2}$ and $t_{3}$ exist and are unique. Then, following the characteristic curves and using (A.11, A.12) and (A.13) one gets

$$
z_{1}\left(\tau_{4}^{\lambda}, x\right)=\left(\frac{1+\frac{\gamma}{4}}{1+\gamma}\right) \alpha_{-}^{2}\left(x_{2}\right)+\left(\frac{1+\frac{\gamma}{4}}{1+\gamma}\right)^{2} \alpha_{-}^{2}\left(x_{3}\right)+\left(\frac{1+\frac{\gamma}{4}}{1+\gamma}\right)^{3} \alpha_{-}^{1}\left(x_{1}\right)
$$

which is quite different from (A.47). In particular, (A.74) implies that the norm of the linear map $\alpha_{-} \mapsto z\left(\tau_{4}^{\lambda}\right)$ is at least $3 / 2$ for $\gamma$ small enough. By (A.47) and (A.46) the norm of this linear map is $(1+(\gamma / 4)) /(1+\gamma)<1$ when $\theta(\lambda)=0$.

Finally, let us deduce Lemma 17 from Lemma 24. Let $(\lambda, \alpha, \beta, b, f) \in \mathcal{H}_{\gamma_{7} \gamma, \gamma}$ with $\gamma \in\left(0, \gamma_{7}\right]$, where $\gamma_{7}>0$ is small and will be specified later on. Let

$$
\tilde{w}=\frac{1}{2}\left((1+\gamma)\left(\beta^{2}(1)-\beta^{1}(1)\right)+b_{1}\left(T^{*}\right)\right)
$$

and let $\tilde{Z}=\left(\tilde{Z}^{1}, \tilde{Z}^{2}\right) \in \mathcal{C}^{0}\left(\left[0, T^{*}\right] \times[0,1]\right)^{2}$ be defined by requiring

$$
\begin{gathered}
\tilde{Z}\left(T^{*}, \cdot\right)=\beta \\
\tilde{Z}_{t}^{1}+\lambda_{1} \tilde{Z}_{x}^{1}=f^{1}, \tilde{Z}_{t}^{2}+\lambda_{2} \tilde{Z}_{x}^{2}=f^{2} \\
\left(1+\frac{\gamma}{4}\right)\left(\tilde{Z}^{2}(t, 0)-\tilde{Z}^{1}(t, 0)\right)+b_{0}(t)=(1+\gamma)\left(\tilde{Z}^{2}(t, 1)-\tilde{Z}^{1}(t, 1)\right)+b_{1}(t)=2 \tilde{w} .
\end{gathered}
$$

The existence of such a $\tilde{Z}$ follows from the compatibility condition (4.47) and (A.75). Let $\bar{Z}=\left(\bar{Z}^{1}, \bar{Z}^{2}\right)$ $\in \mathcal{C}^{0}\left(\left[0, \tau_{0}\right] \times[0,1]\right)^{2}$ be defined by requiring

$$
\begin{gathered}
\bar{Z}(0, \cdot)=\alpha-\tilde{Z}(0, \cdot) \\
\bar{Z}_{t}^{1}+\lambda_{1} \bar{Z}_{x}^{1}=0, \bar{Z}_{t}^{2}+\lambda_{2} \bar{Z}_{x}^{2}=0 \\
\left(1+\frac{\gamma}{4}\right)\left(\bar{Z}^{2}(t, 0)-\bar{Z}^{1}(t, 0)\right)=(1+\gamma)\left(\bar{Z}^{2}(t, 1)-\bar{Z}^{1}(t, 1)\right) \\
\bar{Z}^{2}(t, 0)=\frac{\tau_{0}-t}{\tau_{0}}\left(\alpha^{2}(0)-\tilde{Z}^{2}(0,0)\right)
\end{gathered}
$$


The existence of such a $\bar{Z}$ follows from (4.46) and (A.78). Then, as one easily checks, it suffices to choose

$$
w(t):= \begin{cases}\frac{1+\gamma}{2}\left(\bar{Z}^{2}(t, 1)-\bar{Z}^{1}(t, 1)\right)+\tilde{w} & \text { if } t \in\left[0, \tau_{0}\right], \\ \mathbf{W}_{\gamma}^{0}\left(\lambda,\left(\bar{Z}^{1}\left(\tau_{0}, \cdot\right), \bar{Z}^{2}\left(\tau_{0}, \cdot\right)\right)\right)(t)+\tilde{w} & \text { if } t \in\left[\tau_{0}, T^{*}\right] .\end{cases}
$$

Remark 27. Instead of Lemma 17, Lemma 25 can be used directly to build another map $\mathbf{F}$ which has also a fixed point and such that the existence of a fixed point to $\mathbf{F}$ implies Proposition 5 . We have preferred to present Lemma 25 as an intermediate step just because we thought it was clearer that way.

We thank Pierre Rouchon for having attracted our attention to this controllability problem and for useful discussions.

\section{REFERENCES}

[1] J.-M. Coron, Global asymptotic stabilization for controllable systems without drift. Math. Control Signals Systems 5 (1992) 295-312.

[2] J.-M. Coron, Contrôlabilité exacte frontière de l'équation d'Euler des fluides parfaits incompressibles bidimensionnels. $C$. $R$. Acad. Sci. Paris 317 (1993) 271-276.

[3] J.-M. Coron, On the controllability of 2-D incompressible perfect fluids. J. Math. Pures Appl. 75 (1996) 155-188.

[4] J.-M. Coron, On the controllability of the 2-D incompressible Navier-Stokes equations with the Navier slip boundary conditions. ESAIM: COCV 1 (1996) 35-75.

[5] J.-M. Coron and A. Fursikov, Global exact controllability of the 2D Navier-Stokes equations on a manifold without boundary. Russian J. Math. Phys. 4 (1996) 429-448.

[6] R. Courant and D. Hilbert, Methods of mathematical physics, II. Interscience publishers, John Wiley \& Sons, New York London Sydney (1962).

[7] L. Debnath, Nonlinear water waves. Academic Press, San Diego (1994).

[8] F. Dubois, N. Petit and P. Rouchon, Motion planning and nonlinear simulations for a tank containing a fluid, ECC 99.

[9] A.V. Fursikov and O.Yu. Imanuvilov, Exact controllability of the Navier-Stokes and Boussinesq equations. Russian Math. Surveys. 54 (1999) 565-618.

[10] O. Glass, Contrôlabilité exacte frontière de l'équation d'Euler des fluides parfaits incompressibles en dimension 3. C.R.Acad. Sci. Paris Sér. I 325 (1997) 987-992.

[11] O. Glass, Exact boundary controllability of 3-D Euler equation. ESAIM: COCV 5 (2000) 1-44.

[12] L. Hörmander, Lectures on nonlinear hyperbolic differential equations. Springer-Verlag, Berlin Heidelberg, Math. Appl. 26 (1997).

[13] Th. Horsin, On the controllability of the Burgers equation. ESAIM: COCV 3 (1998) 83-95.

[14] J.-L. Lions, Are there connections between turbulence and controllability?, in 9th INRIA International Conference. Antibes (1990).

[15] J.-L. Lions, Exact controllability for distributed systems. Some trends and some problems, in Applied and industrial mathematics, Proc. Symp., Venice/Italy 1989. D. Reidel Publ. Co. Math. Appl. 56 (1991) 59-84.

[16] J.-L. Lions, On the controllability of distributed systems. Proc. Natl. Acad. Sci. USA 94 (1997) 4828-4835.

[17] J.-L. Lions and E. Zuazua, Approximate controllability of a hydro-elastic coupled system. ESAIM: COCV 1 (1995) 1-15.

[18] J.-L. Lions and E. Zuazua, Exact boundary controllability of Galerkin's approximations of Navier-Stokes equations. Ann. Sc. Norm. Super. Pisa Cl. Sci. IV 26 (1998) 605-621.

[19] Li Ta Tsien and Yu Wen-Ci, Boundary value problems for quasilinear hyperbolic systems. Duke university, Durham, Math. Ser. V (1985).

[20] A. Majda, Compressible fluid flow and systems of conservation laws in several space variables. Sringer-Verlag, New York Berlin Heidelberg Tokyo, Appl. Math. Sci. 53 (1984).

[21] N. Petit and P. Rouchon, Dynamics and solutions to some control problems for water-tank systems. Preprint, CIT-CDS 00-004.

[22] A.J.C.B. de Saint-Venant, Théorie du mouvement non permanent des eaux, avec applications aux crues des rivières et à l'introduction des marées dans leur lit. C. R. Acad. Sci. Paris 53 (1871) 147-154.

[23] D. Serre, Systèmes de lois de conservations, I et II. Diderot Éditeur, Arts et Sciences, Paris, New York, Amsterdam (1996).

[24] E.D. Sontag, Control of systems without drift via generic loops. IEEE Trans. Automat. Control. 40 (1995) 1210-1219. 